\title{
Sex differences in human mate preferences: Evolutionary hypotheses tested in 37 cultures
}

\author{
David M. Buss \\ Department of Psychology, University of Michigan, Ann Arbor, MI \\ 48109-1346 \\ Electronic mail: david buss@um.cc.umich.edu
}

\begin{abstract}
Contemporary mate preferences can provide important clues to human reproductive history. Little is known about which characteristics people value in potential mates. Five predictions were made about sex differences in human mate preferences based on evolutionary conceptions of parental investment, sexual selection, human reproductive capacity, and sexual asymmetries regarding certainty of paternity versus maternity. The predictions centered on how each sex valued earning capacity, ambitionindustriousness, youth, physical attractiveness, and chastity. Predictions were tested in data from 37 samples drawn from 33 countries located on six continents and five islands (total $N=10,047$ ). For 27 countries, demographic data on actual age at marriage provided a validity check on questionnaire data. Females were found to value cues to resource acquisition in potential mates more highly than males. Characteristics signaling reproductive capacity were valued more by males than by females. These sex differences may reflect different evolutionary selection pressures on human males and females; they provide powerful cross-cultural evidence of current sex differences in reproductive strategies. Discussion focuses on proximate mechanisms underlying mate preferences, consequences for human intrasexual competition, and the limitations of this study.
\end{abstract}

Keywords: assortative mating; cultural differences; evolution; mate preferences; reproductive strategy; sex differences; sexual selection; sociobiology

\section{Introduction}

Mate preferences acquire importance in at least three scientific contexts. First, they can affect the current direction of sexual selection by influencing who is differentially excluded from and included in mating (Darwin 1871). Favored mate characteristics that show some heritability will typically be represented more frequently in subsequent generations. Individuals lacking favored characteristics tend to become no one's ancestors (Thornhill \& Thornhill 1983). Second, current mate preferences may reflect prior selection pressures, thus providing important clues to a species' reproductive history. Third, mate preferences can exert selective pressures on other components of the mating system. In the context of intrasexual competition, for example, tactics used to attract and retain mates should be strongly influenced by the mate preferences expressed by members of the opposite sex (Buss 1988). Because of the powerful reproductive consequences of preferential mating, it is reasonable to assume that mate preferences will depart from randomness and evolve through sexual selection (Darwin $1859 ; 1871$; Fisher 1930). This assumption, first advanced by Darwin, has been documented empirically for a variety of nonhuman species (e.g., Bateson 1983; Majerus 1986).

In spite of the importance of mate preferences, little is known about precisely which characteristics in potential mates are valued by human males and females (Buss 1985; Thiessen \& Gregg 1980). Particularly lacking are good cross-cultural data. Cross-cultural studies become crucial for testing evolution-based hypotheses that posit speciestypical or sex-typical mate preferences. Recent theoretical work by Trivers (1972), Williams (1975), Symons (1979), and Buss (1987) provides a foundation from which specific evolutionary hypotheses about mate preferences can be derived. [See also multiple book review of Symons's Evolution of Human Sexuality, BBS 3(2) 1980 and Hartung's "Matrilineal Inheritance" BBS 8(4) 1985.]

\subsection{Predictions from parental investment and sexual selection theory}

Trivers (1972) posits that sexual selection is driven in part by different levels of investment by males and females in their offspring (Bateman 1948). In humans and other mammals, male parental investment tends to be less than female parental investment (Fisher 1930; Trivers 1972; Williams 1975). Mammalian fertilization occurs internally within females, as does gestation. A copulation that requires minimal male investment can produce a 9month investment by the female that is substantial in terms of time, energy, resources, and foreclosed alternatives.

Investment, of course, does not begin with fertilization, nor does it end with parturition. Trivers describes 
several forms of male investment. Males may provide mates with food, find or defend territories, defend the female against aggressors, and feed and protect the young. Human males may also provide opportunities for learning, they may transfer status, power, or resources, and they may aid their offspring in forming reciprocal alliances. These forms of male investment, when provided, tend to decrease the investment disparities between males and females (Trivers 1972 , p. 142).

Trivers's theory proposes that the sex investing more in offspring (typically the female) will be selected to exert stronger preferences about mating partners. This greater choosiness by the more heavily investing sex exists because greater reproductive costs are associated with indiscriminate mating and greater benefits are associated with exerting a choice. The costs of less discriminating mating will be lower for the sex investing less and the benefits will be greater. In species where investment in offspring by males and females is equivalent, the sexes are expected to be equally discriminating in their choice of mating partners (Trivers 1985).

What mate characteristics might be predicted on theoretical grounds in the selection preferences of females? In species with male parental investment, such as Homo sapiens (Alexander \& Noonan 1979), females should seek to mate with males who have the ability and willingness to provide resources related to parental investment such as food, shelter, territory, and protection. Trivers's prediction should apply only in contexts where resources can be accrued, monopolized, and defended, where males tend to control such resources, and where male variance in resource acquisition is sufficiently high (Emlen \& Oring 1977; Trivers 1972). The hypothesis that females will mate preferentially with males bearing greater gifts, holding better territories, or displaying higher rank has been confirmed empirically in many nonhuman species (Calder 1967; Lack 1940; Trivers 1985; see also Betzig et al. 1988).

These resources can provide (a) immediate material advantage to the female and her offspring, (b) enhanced reproductive advantage for offspring through acquired social and economic benefits, and (c) genetic reproductive advantage for the female and her offspring if variation in the qualities that lead to resource acquisition is partly heritable.

Among humans, resources typically translate into earning capacity. This suggests that females will value characteristics in potential mates that are associated with increased earning capacity, such as ambition and industriousness (Barron 1963; Willerman 1979). These premises, combined with conditions of resource defensibility and high variance in male resource acquisition, produce a specific prediction: Females, more than males, should value attributes in potential mates such as ambition, industriousness, and earning capacity that signal the possession or likely acquisition of resources.

\subsection{Predictions based on fertility and reproductive value}

For males more than for females, reproduction is limited by access to reproductively valuable or fertile mates (Symons 1979; Trivers 1972; Williams 1975). Reproductive value is defined actuarially in units of expected future reproduction - the extent to which persons of a given age and sex will contribute, on average, to the ancestry of future generations (Fisher 1930). Fertility is defined as the probability of present reproduction. In human females, reproductive value typically peaks in the midteens and declines monotonically thereafter with age. Fertility typically peaks in the early 20 s and shows a similar decrement with age (Thornhill \& Thornhill 1983). The difference between fertility and reproductive value may be illustrated by contrasting two females, aged 13 and 23 . The younger female would have higher reproductive value than the older one because, actuarially, her future reproduction is expected to be higher. In contrast, the 23-year-old female would be more fertile than the 13year-old because the current probability of reproduction is higher for the 23-year-old.

Both fertility and reproductive value differ across cultures and are affected by factors such as cultural norms, contraceptive practices, and differences in agespecific mortality. In all cultures, however, female fertility and reproductive value are strongly age-dependent (Williams 1975). Thus, age provides a powerful cue to female reproductive capacity - a cue that can be inferred through physical and behavioral attributes or with veridical use of counting systems.

Males should prefer attributes in potential mates associated with reproductive value or fertility, depending on whether males in human evolutionary history have tended to seek long-term or short-term mating partners (Buss 1987; Symons 1979; Williams 1975). Specifically, if males in our evolutionary past have tended to seek short-term mating partners, selection should have favored male preferences for females in their early 20 s who show cues that are positively correlated with fertility. If males in our evolutionary past have tended to seek long-term mating partners, selection should have favored preferences for females in their mid-teens who show cues indicative of high reproductive value. Evolutionary theorists differ on which of these hypotheses they judge to be most likely. Symons (1979) argues that males have been selected to find most attractive those females of high reproductive value. Williams (1975), in contrast, predicts a compromise preference between reproductive value and fertility due to the existence of both long-term mating bonds and some possibility of divorce and extrapair matings.

Features of physical appearance associated with youth - such as smooth skin, good muscle tone, lustrous hair, and full lips - and behavioral indicators of youth - such as high energy level and sprightly gait - have been hypothesized to provide the strongest cues to female reproductive capacity (Symons 1979; Williams 1975). Sexual attraction and standards of beauty are hypothesized to have evolved to correspond to these features. On this account, males failing to prefer females possessing attributes that signal high reproductive capacity would, on average, leave fewer offspring than would males who do prefer to mate with females displaying these attributes.

Female reproductive success, in contrast to male reproductive success, is not as closely linked with obtaining fertile mates. Male fertility, to the degree that it is valued by females, is less steeply age-graded from puberty on than is female fertility and therefore cannot be assessed as accurately from physical appearance. Physical appearance, therefore, should be less central to female mate 
preferences than to male mate preferences. These premises lead to specific predictions: Males, more than females, will value relative youth and physical attractiveness in potential mates because of their links with fertility and reproductive value.

Predicting that males will value physical attractiveness in females because of its association with reproductive capacity does not negate or deny the existence of cultural and other determinants of standards for attractiveness. Ford and Beach (1951) have documented cultural variability in standards for female attractiveness along the dimensions of plump versus slim body build, light versus dark skin, and emphasis on particular features such as the eyes, ears, or genitals. Symons (1979) suggested that regularity of features, proximity to the population average, and association with status might also have an important influence on attractiveness standards (see also Buss 1987).

The predicted sex differences in mate preferences for youth and physical attractiveness, however, are expected to transcend cultural variations and other determinants of beauty standards. The physical and behavioral cues that signal youth and health and are regarded as attractive should be linked with reproductive capacity among human females in all cultures. These sex differences are predicted to be species-typical among Homo sapiens, despite cross-cultural variations in absolute age preferences, the presence or absence of counting systems to mark age, or culture-specific criteria for female attractiveness that are not linked with reproductive capacity.

\subsection{Prediction based on paternity probability}

In mating systems where males invest parentally, selection should favor males who act to insure that their investment is directed toward their own offspring and not the offspring of another male. Sexual jealousy is one mechanism that has been proposed to increase paternity probability (Daly et al. 1982). Male sexual jealousy presumably functions to guard a mate and to dissuade intrasexual competitors, thus lowering the likelihood of alien insemination. Daly et al. (1982) and Daly \& Wilson (1988) present compelling evidence that many homicides and much male violence stem from male sexual jealousy.

Another possible paternity probability mechanism is valuation of chastity in a potential mate (Dickemann 1981). Males who preferred chaste females in our environment of evolutionary adaptedness, ceteris paribus, presumably enjoyed greater reproductive success than males who were indifferent to the sexual contact that a potential mate had with other males. Prior to the use of modern contraceptive devices, chastity of a potential mate would provide a cue to paternity confidence. Assuming some temporal stability to behavioral proclivities, chastity would also provide a cue to the future fidelity of a selected mate. A male failing to express such a preference would risk wasting the time and effort involved in courtship and would risk investing in offspring that were not his (Daly \& Wilson 1983; Dickemann 1981).

The association between chastity and probability of parenthood, however, shows a sexual asymmetry. In our environment of evolutionary adaptedness, maternity was never in doubt. A female could be sure that her putative children were her own, regardless of the prior sexual experiences of her mate. This sexual asymmetry yields a specific prediction: Males will value chastity in a potential mate more than will females. Evidence limited to a few cultures exists regarding the importance of a mate's lack of prior sexual experience in mate preferences (Borgerhoff Mulder 1988; Dickemann 1981).

It should be noted that this predicted sex difference would be compromised if prior sexual experience by a male provided a cue that signaled diversion of resources away from the female and her offspring (Buss 1988b). To the degree that prior sexual experience by males provides this cue, females should also value chastity in a potential mate.

In sum, three clusters of sex differences in mate preferences were predicted, based on an evolutionary account of differing male and female reproductive strategies. A woman's "mate value" (Symons 1987a) should be determined more by her reproductive capacity. Youth and physical appearance, as powerful cues to this capacity, should be more highly valued by men. Chastity should also be valued because it functions to increase a male's probability of paternity. A man's "mate value" is determined less by fertility and more by the external resources he can provide. Characteristics indicative of one's potential to provide resources, such as earning capacity, ambition, and industriousness, should receive more emphasis in female mate preferences. The following study was designed to test these hypotheses in 37 cultures differing widely in ecology, location, racial and ethnic composition, religious orientation, political inclination, and nature of mating system.

\section{Methods}

\subsection{Samples}

Thirty-seven samples were obtained from 33 countries located on six continents and five islands, with a total $N$ of 10,047 (see Table 1). The samples range in mean age from 16.96 (New Zealand) to 28.71 (West Germany), with an overall unit-weighted mean of 23.05 . Sample sizes vary from a low of 55 (Iran) to highs of 500 (mainland China), 566 (Taiwan, Republic of China), 630 (Brazil), 1,083 (West Germany), and 1,491 (mainland United States). All samples but one have $N$ s exceeding 100 . The mean sample size for the 37 samples is 272 . Obviously, greater confidence can be placed in the results from the large samples; results from all samples are presented for completeness.

The samples obtained cannot be viewed as representative of the populations in each country. In general, rural, less-educated, and lower levels of socioeconomic status are underrepresented, although there are many exceptions, such as the Soviet Estonian, Gujarati Indian, South African Zulu, Venezuelan, and Santa Catarina Brazilian samples. The 37 samples do represent a tremendous diversity of geographic, cultural, political, ethnic, religious, racial, and economic groups; combined, they are the largest ever obtained on mate preferences.

Sampling techniques varied widely across countries. In Estonia, for example, one subsample consisted of all couples applying for a marriage license at a certain location within a given time span, whereas another Estonian subsample consisted of 200 high school students. The 
Buss: Sex differences

Table 1. Sample sizes and mean ages

\begin{tabular}{|c|c|c|c|c|c|c|c|}
\hline \multirow[b]{2}{*}{ Sample } & \multicolumn{3}{|c|}{ Sample size } & \multicolumn{2}{|c|}{ Age of males } & \multicolumn{2}{|c|}{$\begin{array}{l}\text { Age of } \\
\text { females }\end{array}$} \\
\hline & Total & Male & Female & Mean & SD & Mean & $\mathrm{SD}$ \\
\hline \multicolumn{8}{|l|}{ African } \\
\hline Nigeria & 172 & 117 & 55 & 23.36 & 3.39 & 21.13 & 1.38 \\
\hline S. Africa (whites) & 128 & 47 & 81 & 20.88 & 2.17 & 19.44 & 1.28 \\
\hline S. Africa (Zulu) & 100 & 52 & 48 & 25.30 & 9.40 & 23.53 & 6.18 \\
\hline Zambia & 119 & 70 & 49 & 25.67 & 7.42 & 22.60 & 4.17 \\
\hline \multicolumn{8}{|l|}{ Asian } \\
\hline China & 500 & 265 & 235 & 23.37 & 4.87 & 22.46 & 5.29 \\
\hline India & 247 & 103 & 144 & 30.46 & 12.46 & 24.90 & 10.92 \\
\hline Indonesia & 143 & 88 & 55 & 23.52 & 3.16 & 22.76 & 3.19 \\
\hline Iran & 55 & 28 & 27 & 24.14 & 5.14 & 22.74 & 5.70 \\
\hline Israel (Jewish) & 473 & 205 & 268 & 25.52 & 4.26 & 23.29 & 3.65 \\
\hline Israel (Palestinian) & 109 & 54 & 55 & 23.51 & 3.79 & 21.50 & 3.23 \\
\hline Japan & 259 & 106 & 153 & 20.05 & 1.50 & 19.37 & 0.88 \\
\hline Taiwan & 566 & 288 & 278 & 21.13 & 1.85 & 20.54 & 1.63 \\
\hline \multicolumn{8}{|l|}{ European-Eastern } \\
\hline Bulgaria & 269 & 127 & 142 & 22.28 & 6.16 & 23.06 & 7.04 \\
\hline Estonian S.S.R. & 303 & 153 & 150 & 19.12 & 3.50 & 18.32 & 2.64 \\
\hline Poland & 240 & 122 & 118 & 21.98 & 1.97 & 21.44 & 1.51 \\
\hline Yugoslavia & 140 & 66 & 74 & 21.53 & 1.55 & 20.72 & 1.33 \\
\hline \multicolumn{8}{|l|}{ European-Western } \\
\hline Belgium & 145 & 55 & 90 & 23.80 & 6.23 & 21.38 & 5.49 \\
\hline France & 191 & 100 & 91 & 25.27 & 7.29 & 25.83 & 7.95 \\
\hline Finland & 204 & 55 & 149 & 23.87 & 4.58 & 24.60 & 5.29 \\
\hline Germany-West & 1083 & 530 & 553 & 28.29 & 10.81 & 29.14 & 12.40 \\
\hline Great Britain & 130 & 46 & 84 & 20.87 & 3.92 & 21.09 & 5.38 \\
\hline Greece & 132 & 67 & 65 & 20.72 & 2.50 & 18.71 & 1.46 \\
\hline Ireland & 122 & 55 & 67 & 19.60 & 1.50 & 19.27 & 1.31 \\
\hline Italy & 101 & 46 & 55 & 27.83 & 5.32 & 25.96 & 5.39 \\
\hline Netherlands & 417 & 177 & 240 & 22.74 & 3.86 & 21.65 & 3.31 \\
\hline Norway & 134 & 67 & 67 & 22.25 & 4.10 & 22.46 & 4.46 \\
\hline Spain & 124 & 44 & 80 & 22.89 & 2.58 & 22.75 & 3.59 \\
\hline Sweden & 172 & 89 & 83 & 29.79 & 9.88 & 26.70 & 8.20 \\
\hline \multicolumn{8}{|l|}{ North American } \\
\hline Canada (English) & 101 & 56 & 45 & 20.89 & 2.98 & 23.05 & 6.84 \\
\hline Canada (French) & 105 & 34 & 71 & 26.00 & 6.32 & 25.17 & 8.16 \\
\hline USA (Mainland) & 1491 & 639 & 852 & 19.98 & 3.45 & 20.37 & 4.63 \\
\hline USA (Hawaii) & 179 & 66 & 113 & 23.79 & 7.23 & 22.76 & 6.20 \\
\hline \multicolumn{8}{|l|}{ Oceanian } \\
\hline Australia & 280 & 78 & 202 & 25.06 & 8.50 & 23.12 & 8.38 \\
\hline New Zealand & 151 & 75 & 76 & 17.00 & 0.79 & 16.92 & 0.81 \\
\hline \multicolumn{8}{|l|}{ South American } \\
\hline Brazil & 630 & 275 & 355 & 22.84 & 4.59 & 21.72 & 4.47 \\
\hline Colombia & 139 & 61 & 78 & 25.89 & 6.76 & 24.34 & 6.03 \\
\hline Venezuela & 193 & 95 & 98 & 28.07 & 7.19 & 28.42 & 7.19 \\
\hline Summary & 10,047 & 4,601 & 5,446 & 23.49 & 3.01 & 22.52 & 2.67 \\
\hline
\end{tabular}

Note: $\mathrm{SD}=$ standard deviation.

Venezuelan sample was obtained by contacting every fifth house within each of a series of neighborhoods that varied in socioeconomic class. The South African Zulu sample was rural, and questions were read aloud to some subjects. The West German sample was obtained by mail through newspaper advertisements. The New Zealand samples were drawn from three public high schools, two urban and one rural, with subjects differing widely in socioeconomic level. Many were samples of convenience (e.g., university students) and cannot be viewed as repre- 
sentative. The wide variety of sampling techniques used tends to increase the generality of consistent results that do emerge by minimizing the biasing effects of any particular sampling procedure.

Problems were encountered, and data collection proved difficult and time consuming. In Sweden, many couples do not get married, but instead live together without the official marriage certificate. The instruments had to be modified to reflect this cultural difference. In Nigeria, polygyny is practiced, and so questions had to be added to reflect the possibility of multiple wives. In South Africa, data collection was described as "a rather frightening experience" due to the political turmoil and its violent ramifications. In several countries, mailing the data was delayed for many months, pending approval of central government committees. In one country, after data collection was nearly completed, the study had to be terminated because of a failure to obtain official sanction. Data from this country were never received.

In most cases, data were collected by native residents within each country and mailed to the United States for statistical analysis. The original protocols were requested, and in most cases these were sent. In a few cases it proved impossible to send the original protocols. In these cases, the raw data were transcribed onto coding sheets and sent to the United States. Research collaborators were blind with respect to the central hypotheses.

\subsection{Measures}

2.2.1. Factors in choosing a mate. This instrument consisted of three parts. The first part requested biographical data, including age, sex, religion, marital status, number of brothers, and number of sisters. The second section requested information on the age at which the respondent preferred to marry, the age difference the respondent preferred to have between self and spouse, who the respondent preferred to be older (self or spouse), and how many children were desired.

The third section requested subjects to rate each of 18 characteristics (e.g., dependable character, sociability, chastity, intelligence) on how important or desirable it would be in choosing a mate. A four-point scale was used, ranging from " 3 " (indispensable) to " 0 " (irrelevant or unimportant). The 18 characteristics were drawn from a previously developed instrument used widely within the United States over the past 50 years (Hill 1945; Hudson \& Henze 1969; McGinnis 1958). Interspersed among the 18 characteristics were the target variables "good financial prospect," "good looks," "chastity: no previous sexual intercourse," and "ambition and industriousness."

2.2.2. Preferences concerning potential mates. The second instrument was developed from the factor analysis (Buss \& Barnes 1986) of an expanded 76-item instrument (Gough 1973). The highest loading items from this factor analysis were included (e.g., religious, kind and understanding, exciting personality), along with several items to test the specific hypotheses about sex differences in mate preferences. Interspersed among the 13 characteristics were the target variables "good earning capacity" and "physically attractive."

In contrast to the rating procedure used in the first instrument, subjects were requested to rank each charac- teristic on its desirability in a mate. The instructional set was as follows:

Below are listed a set of characteristics. Please rank them on their desirability in someone you might marry. Give a " 1 " to the most desirable characteristic in a potential mate; a " 2 " to the second most desirable characteristic in a potential mate; a " 3 " to the third most desirable characteristic, and so on down to " 13 "

for the 13th most desirable characteristic in a potential mate.

In sum, two instruments were used, each containing target variables to test the key predictions. They differed in context (presence of other items) and scaling procedure (rating vs. ranking), permitting a partial test of the generality of the findings across methods.

2.2.3. Translations. Instructions were provided to each research collaborator for translating the two instruments into the appropriate language for their sample. These included the use of three bilingual speakers who, respectively, (a) translated from English to the native language, (b) back-translated from the native language to English, and (c) resolved discrepancies between the first two translators. Instructions were provided to make all terms "sex neutral" in the sense of being equally applicable to males and females. The phrase "physically attractive," for example, could be applied to either sex, whereas "handsome" and "beautiful" were considered sex-linked and were therefore not used.

\section{Results}

\subsection{Earning potential and ambltion-industriousness}

To conserve space, only data from the rated variables are presented in tabular form. Discrepancies between parallel tests using the rating and ranking instruments are noted in the text and with asterisks in the tables. Tables presenting the full parallel analyses for the ranking instrument are available from the author on request.

Table 2 shows the means, standard deviations, $t$-tests for sex differences, and significance levels for valuation of the rated variable "good financial prospect" for each of the 37 samples. Samples vary considerably in how much this mate characteristic is valued, ranging from quite high (Indonesia, Nigeria, Zambia) to quite low (South African Zulu, Netherlands, Great Britain). In general, South American, North American, Asian, and African samples valued earning capacity more than did Western European samples, although there are important variations among samples within each continent.

In 36 of 37 samples, the predicted sex difference emerged - females valued "good financial prospect" in a potential mate more highly than males did. The sole exception was the sample from Spain, which showed the predicted direction of the sex difference, but not significantly so. The ranked variable "good earning capacity" similarly did not show a significant sex difference for the Spanish sample. Whether this lack of significant sex difference is due to particulars of the Spanish mating system, features of the broader socioecology, or chance sample fluctuation must await replication. In sum, with the exception of the Spanish sample, the predicted sex difference in preferences for mates with good earning 
Buss: Sex differences

Table 2. Good financial prospect

\begin{tabular}{|c|c|c|c|c|c|c|}
\hline \multirow[b]{2}{*}{ Sample } & \multicolumn{2}{|c|}{ Males } & \multicolumn{2}{|c|}{ Females } & \multirow[b]{2}{*}{$t$-test } & \multirow[b]{2}{*}{ Sig. } \\
\hline & Mean & SD & Mean & SD & & \\
\hline \multicolumn{7}{|l|}{ African } \\
\hline Nigeria & 1.37 & 0.82 & 2.30 & 0.76 & -7.00 & .000 \\
\hline S. Africa (whites) & 0.94 & 0.78 & 1.73 & 0.78 & -5.58 & .000 \\
\hline S. Africa (Zulu) & 0.70 & 0.87 & 1.14 & 0.80 & -2.61 & .006 \\
\hline Zambia & 1.46 & 0.90 & 2.33 & 0.62 & -6.35 & .000 \\
\hline \multicolumn{7}{|l|}{ Asian } \\
\hline China & 1.10 & 0.98 & 1.56 & 0.94 & -5.34 & .000 \\
\hline India & 1.60 & 0.96 & 2.00 & 0.69 & -3.63 & .000 \\
\hline Indonesia & 1.42 & 0.87 & 2.55 & 0.57 & -9.46 & .000 \\
\hline Iran & 1.25 & 1.04 & 2.04 & 0.85 & -3.06 & .002 \\
\hline Israel (Jewish) & 1.31 & 1.01 & 1.82 & 0.87 & -5.58 & .000 \\
\hline Israel (Palestinian) & 1.28 & 1.05 & 1.67 & 0.92 & -2.05 & .023 \\
\hline Japan & 0.92 & 0.75 & 2.29 & 0.58 & -15.97 & .000 \\
\hline Tajwan & 1.25 & 0.81 & 2.21 & 0.70 & -15.16 & .000 \\
\hline \multicolumn{7}{|l|}{ European-Eastern } \\
\hline Bulgaria & 1.16 & 0.94 & 1.64 & 0.91 & -4.29 & .000 \\
\hline Estonian S.S.R. & 1.31 & 0.86 & 1.51 & 0.85 & -2.06 & .025 \\
\hline Poland & 1.09 & 0.82 & 1.74 & 0.80 & -6.18 & .000 \\
\hline Yugoslavia & 1.27 & 0.76 & 1.66 & 0.75 & -3.07 & .002 \\
\hline \multicolumn{7}{|l|}{ European-Western } \\
\hline Belgium & 0.95 & 0.87 & 1.36 & 0.88 & -2.74 & .004 \\
\hline France & 1.22 & 0.97 & 1.68 & 0.92 & -3.35 & .001 \\
\hline Finland & 0.65 & 0.76 & 1.18 & 0.84 & -4.10 & .000 \\
\hline Germany-West & 1.14 & 0.88 & 1.81 & 0.93 & -10.19 & .000 \\
\hline Great Britain & 0.67 & 0.63 & 1.16 & 0.78 & -3.65 & .000 \\
\hline Greece & 1.16 & 0.95 & 1.92 & 0.78 & -4.97 & .000 \\
\hline Ireland & 0.82 & 0.95 & 1.67 & 0.77 & -5.51 & .000 \\
\hline Italy & 0.87 & 0.69 & 1.33 & 0.80 & -3.06 & .002 \\
\hline Netherlands & 0.69 & 0.81 & 0.94 & 0.84 & -3.00 & .002 \\
\hline Norway & 1.10 & 0.84 & 1.42 & 0.97 & -2.03 & .023 \\
\hline Spain & 1.25 & 0.94 & 1.39 & 0.89 & -0.80 & ns \\
\hline Sweden & 1.18 & 0.90 & 1.75 & 0.75 & -4.44 & .000 \\
\hline \multicolumn{7}{|l|}{ North American } \\
\hline Canada (English) & 1.02 & 0.82 & 1.91 & 0.76 & -5.61 & .000 \\
\hline Canada (French) & 1.47 & 0.83 & 1.94 & 0.63 & -3.25 & .001 \\
\hline USA (Mainland) & 1.08 & 0.88 & 1.96 & 0.82 & -20.00 & .000 \\
\hline USA (Hawaii) & 1.50 & 0.81 & 2.10 & 0.72 & -5.10 & .000 \\
\hline \multicolumn{7}{|l|}{ Oceanian } \\
\hline Australia & 0.69 & 0.73 & 1.54 & 0.80 & -8.47 & .000 \\
\hline New Zealand & 1.35 & 0.97 & 1.63 & 0.75 & -2.03 & .022 \\
\hline \multicolumn{7}{|l|}{ South American } \\
\hline Brazil & 1.24 & 0.89 & 1.91 & 0.78 & -9.91 & .000 \\
\hline Colombia & 1.72 & 0.90 & 2.21 & 0.75 & -3.47 & .001 \\
\hline Venezuela & 1.66 & 0.96 & 2.26 & 0.78 & -4.72 & .000 \\
\hline
\end{tabular}

Note: Potential mean values can range from 0 (unimportant) to 3 (indispensable). Sig. = significance; $n s=$ not significant.

potential was found across widely varying cultures, typically at a high level of statistical significance.

Table 3 shows analogous results for valuation of "ambition and industriousness." Across both sexes, the Nigerian, Zulu, Chinese, Taiwanese, Estonian, Palestinian, Colombian, and Venezuelan samples placed particularly high value on this mate characteristic. In no sample was ambition-industriousness rated low. Samples from the Netherlands, Great Britain, West Germany, and Finland, however, expressed less preference for this mate characteristic than did other samples.

Thirty-four of the 37 samples (92\%) for ambitionindustriousness were in the predicted direction, with females expressing a higher valuation than males. In 29 
Table 3. Ambition and industriousness

\begin{tabular}{|c|c|c|c|c|c|c|}
\hline \multirow[b]{2}{*}{ Sample } & \multicolumn{2}{|c|}{ Males } & \multicolumn{2}{|c|}{ Females } & \multirow[b]{2}{*}{$t$-test } & \multirow[b]{2}{*}{ Sig. } \\
\hline & Mean & SD & Mean & $\mathrm{SD}$ & & \\
\hline \multicolumn{7}{|l|}{ African } \\
\hline Nigeria & 2.25 & 0.68 & 2.61 & 0.56 & -3.49 & .001 \\
\hline S. Africa (whites) & 1.73 & 0.84 & 2.16 & 0.70 & -3.14 & .001 \\
\hline S. Africa (Zulu) & 2.41 & 0.81 & 2.10 & 0.73 & 2.02 & .023 \\
\hline Zambia & 1.97 & 0.92 & 2.14 & 0.75 & -1.06 & ns \\
\hline \multicolumn{7}{|l|}{ Asian } \\
\hline China & 2.22 & 0.85 & 2.63 & 0.59 & -6.41 & .000 \\
\hline India & 1.79 & 0.86 & 2.44 & 0.76 & -6.31 & .000 \\
\hline Indonesia & 1.97 & 0.73 & 2.29 & 0.62 & -2.70 & .004 \\
\hline Iran & 2.68 & 0.55 & 2.81 & 0.48 & -0.98 & ns \\
\hline Israel (Jewish) & 1.78 & 0.99 & 2.43 & 0.71 & -7.66 & .000 \\
\hline Israel (Palestinian) & 2.28 & 0.76 & 2.58 & 0.71 & -2.15 & .017 \\
\hline Japan & 1.92 & 0.71 & 2.37 & 0.62 & -5.53 & .000 \\
\hline Taiwan & 2.24 & 0.73 & 2.81 & 0.42 & -11.31 & .000 \\
\hline \multicolumn{7}{|l|}{ European-Eastern } \\
\hline Bulgaria & 1.67 & 0.91 & 2.15 & 0.81 & -4.63 & .000 \\
\hline Estonian S.S.R. & 2.31 & 0.68 & 2.46 & 0.64 & -2.06 & .020 \\
\hline Poland & 1.93 & 0.84 & 2.29 & 0.72 & -3.49 & .001 \\
\hline Yugoslavia & 1.82 & 0.72 & 2.24 & 0.74 & -3.44 & .001 \\
\hline \multicolumn{7}{|l|}{ European-Western } \\
\hline Belgium & 1.67 & 0.82 & 1.97 & 0.87 & -2.01 & .023 \\
\hline France & 1.75 & 1.02 & 2.00 & 0.90 & -1.79 & .037 \\
\hline Finland & 1.44 & 0.83 & 1.56 & 0.73 & -1.07 & ns \\
\hline Germany-West & 1.40 & 0.81 & 1.66 & 0.87 & -4.23 & .000 \\
\hline Great Britain & 1.15 & 0.70 & 1.59 & 0.90 & -2.84 & .003 \\
\hline Greece & 1.96 & 0.94 & 2.25 & 0.90 & -1.81 & .037 \\
\hline Ireland & 1.44 & 0.88 & 1.76 & 0.81 & -2.10 & .019 \\
\hline Italy & 1.63 & 0.85 & 2.07 & 0.94 & -2.46 & .008 \\
\hline Netherlands & 1.28 & 0.97 & 1.41 & 0.93 & -1.35 & ns \\
\hline Norway & 1.60 & 0.80 & 1.70 & 0.87 & -0.72 & ns \\
\hline Spain & 1.73 & 0.90 & 1.69 & 0.98 & 0.22 & ns \\
\hline Sweden & 1.97 & 0.78 & 2.04 & 0.76 & -0.60 & ns \\
\hline \multicolumn{7}{|l|}{ North American } \\
\hline Canada (English) & 1.82 & 0.69 & 2.32 & 0.71 & -3.53 & .001 \\
\hline Canada (French) & 1.79 & 0.85 & 2.08 & 0.75 & -1.78 & .039 \\
\hline USA (Mainland) & 1.84 & 0.76 & 2.45 & 0.61 & -16.66 & .000 \\
\hline USA (Hawaii) & 1.95 & 0.76 & 2.24 & 0.65 & -2.66 & .005 \\
\hline \multicolumn{7}{|l|}{ Oceanian } \\
\hline Australia & 1.38 & 0.92 & 1.82 & 0.77 & -3.69 & .000 \\
\hline New Zealand & 1.57 & 0.76 & 1.86 & 0.53 & -2.64 & .005 \\
\hline \multicolumn{7}{|l|}{ South American } \\
\hline Brazil & 1.70 & 0.90 & 2.21 & 0.82 & -7.25 & .000 \\
\hline Colombia & 2.36 & 0.80 & 2.24 & 0.90 & 0.80 & ns \\
\hline Venezuela & 2.18 & 0.89 & 2.42 & 0.75 & -2.03 & .022 \\
\hline
\end{tabular}

Note: Potential mean values can range from 0 (unimportant) to 3 (indispensable).

samples $(78 \%)$, the sex difference was statistically significant beyond the .05 level. Three samples - Colombian, Spanish, and South African Zulu - show the opposite sex difference, significant only in the Zulu sample. According to the research collaborator who collected the Zulu data, it is considered women's work to build the house, fetch water, and perform other arduous physical tasks, whereas men often travel from their rural homes to urban centers for work. This local division of labor might account for the sex difference reversal among the Zulu. In sum, moder- 
Buss: Sex differences

Table 4. Age difference preferred between self and spouse

\begin{tabular}{|c|c|c|c|c|c|c|c|}
\hline \multirow[b]{2}{*}{ Sample } & \multicolumn{2}{|c|}{ Males } & \multicolumn{2}{|c|}{ Females } & \multirow[b]{2}{*}{$t$-test } & \multirow[b]{2}{*}{ Sig. } & \multirow{2}{*}{$\begin{array}{c}\text { Actual } \\
\text { age } \\
\text { diff. }\end{array}$} \\
\hline & Mean & SD & Mean & SD & & & \\
\hline \multicolumn{8}{|l|}{ African } \\
\hline Nigeria & -6.45 & 5.04 & 4.90 & 2.17 & 21.99 & .000 & - \\
\hline S. Africa (whites) & -2.30 & 2.19 & 3.50 & 2.23 & 13.38 & .000 & 3.13 \\
\hline S. Africa (Zulu) & -3.33 & 2.31 & 3.76 & 3.68 & 10.80 & .000 & 2.38 \\
\hline Zambia & -7.38 & 6.39 & 4.14 & 1.99 & 12.22 & .000 & - \\
\hline \multicolumn{8}{|l|}{ Asian } \\
\hline China & -2.05 & 2.47 & 3.45 & 1.73 & 29.06 & .000 & - \\
\hline India & -3.06 & 2.55 & 3.29 & 1.96 & 19.07 & .000 & - \\
\hline Indonesia & -2.72 & 4.41 & 4.69 & 1.87 & 13.29 & .000 & - \\
\hline Iran & -4.02 & 1.62 & 5.10 & 1.79 & 17.98 & .000 & - \\
\hline Israel (Jewish) & -2.88 & 3.82 & 3.95 & 4.90 & 14.13 & .000 & 3.57 \\
\hline Israel (Palestinian) & -3.75 & 1.99 & 3.71 & 1.86 & 6.66 & .000 & 3.57 \\
\hline Japan & -2.37 & 2.29 & 3.05 & 1.62 & 20.98 & .000 & 2.92 \\
\hline Taiwan & -3.13 & 2.29 & 3.78 & 1.98 & 36.76 & .000 & 3.50 \\
\hline \multicolumn{8}{|l|}{ European-Eastern } \\
\hline Bulgaria & -3.13 & 2.87 & 4.18 & 2.61 & 21.35 & .000 & 3.54 \\
\hline Estonian S.S.R. & -2.19 & 2.58 & 2.85 & 1.52 & 22.69 & .000 & 2.49 \\
\hline Poland & -2.85 & 2.94 & 3.38 & 3.02 & 14.66 & .000 & 2.10 \\
\hline Yugoslavia & -2.47 & 2.29 & 3.61 & 1.98 & 16.29 & .000 & 3.55 \\
\hline \multicolumn{8}{|l|}{ European-Western } \\
\hline Belgium & -2.53 & 5.15 & 2.46 & 2.49 & 5.49 & .000 & 2.37 \\
\hline France & -1.94 & 2.47 & 4.00 & 3.17 & 12.97 & .000 & 2.28 \\
\hline Finland & -0.38 & 3.22 & 2.83 & 2.35 & 5.57 & .000 & 2.30 \\
\hline Germany-West & -2.52 & 3.87 & 3.70 & 3.67 & 20.18 & .000 & 3.19 \\
\hline Great Britain & -1.92 & 3.78 & 2.26 & 2.58 & 6.02 & .000 & 2.61 \\
\hline Greece & -3.36 & 3.20 & 4.54 & 2.55 & 14.98 & .000 & 4.92 \\
\hline Ireland & -2.07 & 1.93 & 2.78 & 1.91 & 12.79 & .000 & 2.17 \\
\hline Italy & -2.76 & 2.77 & 3.24 & 2.41 & 10.85 & .000 & 3.68 \\
\hline Netherlands & -1.01 & 2.51 & 2.72 & 3.01 & 9.82 & .000 & 2.58 \\
\hline Norway & -1.91 & 4.14 & 3.12 & 2.36 & 7.80 & .000 & 2.87 \\
\hline Spain & -1.46 & 2.43 & 2.60 & 4.25 & 5.92 & .000 & 2.45 \\
\hline Sweden & -2.34 & 4.87 & 2.91 & 2.79 & 8.08 & .000 & 2.97 \\
\hline \multicolumn{8}{|l|}{ North American } \\
\hline Canada (English) & -1.53 & 1.93 & 2.72 & 2.01 & 10.15 & .000 & 2.51 \\
\hline Canada (French) & -1.22 & 1.69 & 1.82 & 1.83 & 7.43 & .000 & 2.51 \\
\hline USA (Mainland) & -1.65 & 2.62 & 2.54 & 1.90 & 31.76 & .000 & 2.71 \\
\hline USA (Hawaii) & -1.92 & 2.46 & 3.30 & 3.25 & 11.57 & .000 & - \\
\hline \multicolumn{8}{|l|}{ Oceanian } \\
\hline Australia & -1.77 & 2.34 & 2.86 & 2.72 & 12.16 & .000 & 2.73 \\
\hline New Zealand & -1.59 & 2.47 & 2.91 & 1.85 & 11.66 & .000 & 2.78 \\
\hline \multicolumn{8}{|l|}{ South American } \\
\hline Brazil & -2.94 & 3.35 & 3.94 & 3.23 & 22.06 & .000 & 3.52 \\
\hline Colombia & -4.45 & 3.01 & 4.51 & 2.85 & 16.88 & .000 & 4.53 \\
\hline Venezuela & -2.99 & 3.05 & 3.62 & 3.25 & 13.63 & .000 & 3.47 \\
\hline Mean & -2.66 & & 3.42 & & & & 2.99 \\
\hline
\end{tabular}

Note: Negative values signify preference for a younger mate; positive values signify preference for an older mate. 
ate support was found for the hypothesized sex difference in this cue to resource acquisition, although this difference cannot be considered universal.

\subsection{Age differences}

Table 4 shows the age differences preferred between self and mate. In each of the 37 samples, males prefer mates who are younger, which is consistent with the hypothesis that males value mates with higher reproductive capacity. These sex differences are the largest ones found in this study, showing statistical significance beyond the .0001 level in each of the 37 samples. Do the age preferences males express for females correspond more closely to peak reproductive value (mid-teens) or to peak fertility (early 20s)? By subtracting the mean age difference preferred between males and their mates (2.66 years) from the age at which males prefer to marry (27.49 years), it can be inferred that males in these samples prefer to marry females who are approximately 24.83 years old. This age preference is closer to peak female fertility than to peak reproductive value.

Not specifically predicted, but also consistent across all countries, females prefer mates who are older than they are. Indeed, females appear to prefer a larger age difference ( 3.42 years older) than do males (2.66 years younger). Adding the mean age difference preferred by females to the age at which females prefer to marry $(25.39$ years) yields a preferred mate age of 28.81 years.

The samples vary strikingly in age difference preferences. Nigeria and Zambia are the two countries in which males prefer the largest age difference between self and mate, 6.45 and 7.38 years younger, respectively. These are the only two countries in this study that practice substantial polygyny. In polygynous mating systems, males are typically older when they acquire wives than is the case in monogamous mating systems (Hart \& Pilling 1960; Murdock 1967).

\subsection{Actual age difference at marriage - a validity check}

Two crucial questions can be posed about the validity of the methods and the reality of the preferences indicated by this study: Are self-reported preferences accurate indices of actual preferences? Are mate preferences reflected in actual mating decisions? To begin to address these questions, data were obtained from the most recent Demographic Yearbook (United Nations 1988) and the Demographic Fact Book (Republic of China 1987) on actual age at marriage. Demographic statistics were obtained for 27 of the 33 countries sampled in this study.

Actual age at marriage is not the same variable as preferred age at marriage or preferred mate age. Actual age at marriage is undoubtedly determined by many factors, including personal preferences, parental preferences, preferences exerted by members of the opposite sex, sex ratio, local availability of mates, and perhaps current resource holdings. Nonetheless, personal preferences, if they are to bear the conceptual importance ascribed to them in this study, should be reflected to some degree in actual mating decisions.
Actual age at marriage was estimated from the data presented for each country in the Demographic Yearbook and the Demographic Fact Book. Data in the Yearbook are broken down by age of bride and age of groom within each of a series of 5-year age brackets (e.g. , 15-19; 20-24; 25-29). An estimated mean age of marriage was obtained by taking the mid-point of each of these age ranges and weighting this by the actual number of brides or grooms falling within the range. This must be regarded as an estimate or approximation of actual marriage age.

Several validity checks can be conducted by comparing these data with the preferred age at marriage, the age difference desired between self and mate, and the preferred mate age derived from these variables. Perhaps most central to this article are the comparisons between the age difference desired between self and mate and the actual age difference between marriage partners. These data are shown in Table 4 along with data on preferred age differences.

Across the 27 countries, the actual age differences between men and women at marriage range from 2.17 years (Ireland) to 4.92 years (Greece), all showing the wives to be younger on average than their husbands. The unit-weighted average age difference between husbands and wives across countries is 2.99 years. The present study found that males prefer their marriage partners to be 2.66 years younger on average, whereas females prefer mates to be 3.42 years older. Averaging across the sexes yields a mean preferred age difference of 3.04 years, which corresponds closely to the actual age difference of 2.99 years between spouses. Thus, preferred age differences between spouses are indeed reflected in actual age differences at marriage.

A second validity check can be made by comparing the absolute values of actual age at marriage with (a) preferred age at marriage and (b) preferred mate age. Males in this study indicate an average preferred marriage age of 27.5 years, with a preferred spouse age of 24.8 years. Females express a preference to marry at 25.4 , and a spouse preferred to be 28.8 years old. Both preferred age of marriage and preferred mate age correspond closely in absolute value to the actual mean ages of grooms (28.2) and brides (25.3).

A third and perhaps more subtle validity check may be made across countries by correlating the magnitude of the preferred age difference with the magnitude of the actual age difference. This cross-country correlation is $+.68(p<.001, N=28)$ for males and $+.71(p<.001, N$ $=28$ ) for females. Samples preferring larger age differences indeed reside in countries where actual marriages show larger age differences. Samples from countries preferring smaller age differences inhabit countries where actual marriages show smaller age differences.

Several conclusions may be drawn from these validity checks. First, they provide strong validation for the selfreport method used to obtain age preferences, and by implication, circumstantial validation for the other selfreport measures used in this study. Second, they yield evidence that stated preferences are reflected in actual mating decisions. Third, they provide further support for the evolution-based hypothesis that males both prefer and choose females displaying cues to high reproductive capacity. 
Buss: Sex differences

Table 5. Good looks

\begin{tabular}{|c|c|c|c|c|c|c|}
\hline \multirow[b]{2}{*}{ Sample } & \multicolumn{2}{|c|}{ Males } & \multicolumn{2}{|c|}{ Females } & \multirow[b]{2}{*}{$t$-test } & \multirow[b]{2}{*}{ Sig. } \\
\hline & Mean & SD & Mean & SD & & \\
\hline \multicolumn{7}{|l|}{ African } \\
\hline Nigeria & 2.24 & 0.67 & 1.82 & 0.72 & 3.65 & .000 \\
\hline S. Africa (whites) & 1.58 & 0.65 & 1.22 & 0.65 & 3.05 & .002 \\
\hline S. Africa (Zulu) & 1.17 & 0.80 & 0.88 & 0.68 & 1.94 & .027 \\
\hline Zambia & 2.23 & 0.85 & 1.65 & 0.84 & 3.72 & .000 \\
\hline \multicolumn{7}{|l|}{ Asian } \\
\hline China & 2.06 & 0.62 & 1.59 & 0.68 & 8.17 & .000 \\
\hline India & 2.03 & 0.73 & 1.97 & 0.75 & 0.59 & ns* \\
\hline Indonesia & 1.81 & 0.81 & 1.36 & 0.62 & 3.76 & .000 \\
\hline Iran & 2.07 & 0.73 & 1.69 & 0.68 & 1.97 & .027 \\
\hline Israel (Jewish) & 1.77 & 0.93 & 1.56 & 0.75 & 2.52 & .006 \\
\hline Israel (Palestinian) & 2.38 & 0.60 & 1.47 & 0.81 & 6.72 & .000 \\
\hline Japan & 1.50 & 0.75 & 1.09 & 0.74 & 4.36 & .000 \\
\hline Taiwan & 1.76 & 0.77 & 1.28 & 0.66 & 8.07 & .000 \\
\hline \multicolumn{7}{|l|}{ European-Eastern } \\
\hline Bulgaria & 2.39 & 0.68 & 1.95 & 0.84 & 4.70 & .000 \\
\hline Estonian S.S.R. & 2.27 & 0.69 & 1.63 & 0.70 & 8.10 & .000 \\
\hline Poland & 1.93 & 0.83 & 1.77 & 0.76 & 1.57 & ns* \\
\hline Yugoslavia & 2.20 & 0.66 & 1.74 & 0.72 & 3.86 & .000 \\
\hline \multicolumn{7}{|l|}{ European-Western } \\
\hline Belgium & 1.78 & 0.84 & 1.28 & 0.79 & 3.58 & .000 \\
\hline France & 2.08 & 0.81 & 1.76 & 0.77 & 2.78 & .003 \\
\hline Finland & 1.56 & 0.81 & 0.99 & 0.73 & 4.79 & .000 \\
\hline Germany-West & 1.92 & 0.74 & 1.32 & 0.72 & 11.37 & .000 \\
\hline Great Britain & 1.96 & 0.60 & 1.36 & 0.72 & 4.76 & .000 \\
\hline Greece & 2.22 & 0.69 & 1.94 & 0.77 & 2.14 & .018 \\
\hline Ireland & 1.87 & 0.64 & 1.22 & 0.69 & 5.33 & .000 \\
\hline Italy & 2.00 & 0.70 & 1.64 & 0.83 & 2.36 & .010 \\
\hline Netherlands & 1.76 & 0.72 & 1.21 & 0.72 & 7.81 & .000 \\
\hline Norway & 1.87 & 0.83 & 1.32 & 0.83 & 3.85 & .000 \\
\hline Spain & 1.91 & 0.68 & 1.24 & 0.82 & 4.65 & .000 \\
\hline Sweden & 1.65 & 0.77 & 1.46 & 0.83 & 1.55 & ns* \\
\hline \multicolumn{7}{|l|}{ North American } \\
\hline Canada (English) & 1.96 & 0.50 & 1.64 & 0.71 & 2.55 & .007 \\
\hline Canada (French) & 1.68 & 0.64 & 1.41 & 0.65 & 2.00 & .024 \\
\hline USA (Mainland) & 2.11 & 0.69 & 1.67 & 0.69 & 12.19 & .000 \\
\hline USA (Hawaii) & 2.06 & 0.75 & 1.49 & 0.81 & 4.67 & .000 \\
\hline \multicolumn{7}{|l|}{ Oceanian } \\
\hline Australia & 1.65 & 0.74 & 1.24 & 0.73 & 4.20 & .000 \\
\hline New Zealand & 1.99 & 0.69 & 1.29 & 0.73 & 5.98 & .000 \\
\hline \multicolumn{7}{|l|}{ South American } \\
\hline Brazil & 1.89 & 0.75 & 1.68 & 0.86 & 3.25 & .001 \\
\hline Colombia & 1.56 & 0.79 & 1.22 & 0.75 & 2.63 & .005 \\
\hline Venezuela & 1.76 & 0.90 & 1.27 & 0.98 & 3.64 & .000 \\
\hline
\end{tabular}

Note: ${ }^{*}$ indicates significant in predicted direction on the ranking procedure for variable "physically attractive." 
Buss: Sex differences

Table 6. Chastity: No Previous experience in sexual intercourse

\begin{tabular}{|c|c|c|c|c|c|c|}
\hline \multirow[b]{2}{*}{ Sample } & \multicolumn{2}{|c|}{ Males } & \multicolumn{2}{|c|}{ Females } & \multirow[b]{2}{*}{$t$-test } & \multirow[b]{2}{*}{ Sig. } \\
\hline & Mean & SD & Mean & SD & & \\
\hline \multicolumn{7}{|l|}{ African } \\
\hline Nigeria & 1.22 & 1.10 & 0.51 & 0.72 & 4.97 & .000 \\
\hline S. Africa (whites) & 1.06 & 1.05 & 0.84 & 1.12 & 1.13 & ns \\
\hline S. Africa (Zulu) & 1.17 & 1.06 & 0.31 & 0.62 & 4.82 & .000 \\
\hline Zambia & 1.66 & 1.03 & 0.98 & 1.03 & 3.29 & .001 \\
\hline \multicolumn{7}{|l|}{ Asian } \\
\hline China & 2.54 & 0.82 & 2.61 & 0.77 & -1.03 & ns \\
\hline India & 2.44 & 0.98 & 2.17 & 1.11 & 1.95 & .027 \\
\hline Indonesia & 2.06 & 1.10 & 1.98 & 1.18 & 0.39 & ns \\
\hline Iran & 2.67 & 0.88 & 2.23 & 0.99 & 1.70 & .049 \\
\hline Israel (Jewish) & 0.93 & 1.12 & 0.58 & 0.97 & 3.46 & .001 \\
\hline Israel (Palestinian) & 2.24 & 1.10 & 0.96 & 1.18 & 5.81 & .000 \\
\hline Japan & 1.42 & 1.09 & 0.78 & 0.86 & 5.17 & .000 \\
\hline Taiwan & 2.32 & 0.85 & 2.20 & 0.91 & 1.71 & .040 \\
\hline \multicolumn{7}{|l|}{ European-Eastern } \\
\hline Bulgaria & 0.69 & 0.90 & 0.44 & 0.86 & 2.31 & .011 \\
\hline Estonian S.S.R. & 1.25 & 1.04 & 0.84 & 0.98 & 3.51 & .001 \\
\hline Poland & 1.23 & 1.03 & 0.99 & 1.03 & 1.80 & .031 \\
\hline Yugoslavia & 0.47 & 0.81 & 0.08 & 0.36 & 3.60 & .001 \\
\hline \multicolumn{7}{|l|}{ European-Western } \\
\hline Belgium & 0.67 & 1.02 & 0.38 & 0.72 & 1.89 & .031 \\
\hline France & 0.45 & 0.88 & 0.41 & 0.81 & 0.30 & ns \\
\hline Finland & 0.27 & 0.59 & 0.29 & 0.67 & -0.17 & ns \\
\hline Germany-West & 0.34 & 0.73 & 0.17 & 0.52 & 3.61 & .000 \\
\hline Great Britain & 0.46 & 0.75 & 0.49 & 0.93 & -0.20 & ns \\
\hline Greece & 0.48 & 0.85 & 0.40 & 0.88 & 0.51 & ns \\
\hline Ireland & 1.49 & 1.03 & 1.47 & 1.08 & 0.11 & ns \\
\hline Italy & 0.65 & 0.92 & 0.27 & 0.53 & 2.47 & .008 \\
\hline Netherlands & 0.29 & 0.69 & 0.29 & 0.69 & -0.01 & ns \\
\hline Norway & 0.31 & 0.72 & 0.30 & 0.74 & 0.08 & ns \\
\hline Spain & 0.66 & 0.96 & 0.36 & 0.73 & 1.92 & .029 \\
\hline Sweden & 0.25 & 0.53 & 0.28 & 0.67 & -0.32 & ns \\
\hline \multicolumn{7}{|l|}{ North American } \\
\hline Canada (English) & 0.55 & 0.76 & 0.33 & 0.80 & 1.41 & ns \\
\hline Canada (French) & 0.62 & 0.95 & 0.33 & 0.68 & 1.58 & ns \\
\hline USA (Mainland) & 0.85 & 0.96 & 0.52 & 0.85 & 6.88 & .000 \\
\hline USA (Hawaii) & 0.91 & 0.94 & 0.58 & 0.87 & 2.33 & .011 \\
\hline \multicolumn{7}{|l|}{ Oceanian } \\
\hline Australia & 0.73 & 0.93 & 0.45 & 0.86 & 2.40 & .009 \\
\hline New Zealand & 0.88 & 1.07 & 0.72 & 1.04 & 0.91 & ns \\
\hline \multicolumn{7}{|l|}{ South American } \\
\hline Brazil & 0.93 & 1.08 & 0.36 & 0.78 & 7.32 & .000 \\
\hline Colombia & 1.27 & 1.06 & 0.30 & 0.61 & 6.33 & .000 \\
\hline Venezuela & 0.93 & 1.07 & 0.59 & 0.97 & 2.35 & .010 \\
\hline
\end{tabular}

\subsection{Physical attractiveness}

Table 5 shows the results for the rated variable "good looks." All 37 samples show sex differences in the predicted direction, with 34 significant beyond the .05 level. For those three countries (India, Poland, and Sweden) in which the difference was not significant for "good looks," the sex difference was significant in the predicted direction for the ranked variable "physically attractive." Thus, the hypothesis that males value physical attractiveness in potential mates more than females do is strongly supported by these cross-cultural data.

\subsection{Chastity: No prevlous sexual intercourse}

Table 6 shows the results for the variable of "chastity: no previous experience in sexual intercourse." Cultures in this study vary tremendously in the value placed on this mate characteristic. The samples from China, India, Indonesia, Iran, Taiwan, and Israel (Palestinian Arabs 
only) attach high value to chastity in a potential mate. At the opposite extreme, samples from Sweden, Norway, Finland, the Netherlands, West Germany, and France indicate that prior sexual experience is irrelevant or unimportant in a potential mate. A few subjects even indicated in writing that chastity was undesirable in a potential mate. The Irish sample departs from the other Western European samples in placing moderate emphasis on chastity. Also showing moderate valuation of chastity are samples from Africa, Japan, Poland, and the Soviet republic of Estonia. It is noteworthy that chastity shows greater cross-cultural variability than any other rated variable in this study.

In contrast to the strong cross-cultural consistency of sex differences found with the previous four variables, only $23(62 \%)$ of the samples show significant sex differences in the predicted direction. The remaining 14 samples $(38 \%)$ show no significant sex differences in valuation of chastity. These results provide only moderate support for the evolution-based paternity probability hypothesis. They also yield equally powerful evidence of proximate cultural influences on the degree of importance placed on lack of prior sexual intercourse in a potential mate.

\section{Conclusions}

Each of the five evolution-based predictions received some empirical support from these data. Females value the financial capacity of potential mates more than males do. Ambition and industriousness, cues to resource acquisition, also tend to be valued more heavily by females than by males across cultures. Support was strong for the financial capacity prediction ( 36 of 37 samples), and moderate for the ambition-industriousness prediction ( 29 of 37 samples).

Although these results give powerful support to the evolution-based hypothesis about female preference for males with high providing capacity, the precise functions of this preference remain obscure. By way of comparison, the male arctic tern's ability to bring food to the female during courtship is a good predictor of his ability to feed chicks (Nisbet 1973). Does earning potential provide a similar cue in humans? Or does it provide a cue to increased status, protection, and perhaps even "good genes" (Trivers 1972) that pass to the female's offspring? Future research is needed to identify these functions and to examine characteristics that signal not just the capacity to acquire resources, but the male's willingness to devote those resources to a female and her offspring.

Males value physical attractiveness and relative youth in potential mates more than do females - sex differences that show remarkable generality across cultures. Our demographic data corroborate the preference data, showing that females are younger than males at actual age of marriage. The greater male preference for relative youth and physical attractiveness supports the evolution-based hypothesis about male preference for females showing cues to high reproductive capacity. These findings are especially noteworthy in that they reverse a general trend in these data suggesting that females in a majority of cultures tend to be more exacting in mate preferences across many characteristics. Although cultural variations exist with respect to standards of beauty, these variations apparently do not override sex differences in the importance attached to physical attractiveness.

The male age preference for females of just under 25 years implies that fertility has been a stronger ultimate cause of mate preferences than reproductive value. The fact that this age preference appears to be several years beyond peak fertility, however, suggests that other variables such as similarity (Rushton et al. 1984), compatibility (Murstein 1986), and perhaps maturity might also affect these age preferences. Recent data suggest that fertility may peak later in females than previously thought, perhaps in the mid-twenties (Anderson 1986; Short 1976). If these recent estimates are confirmed, then male age preferences may turn out to be closely calibrated with female fertility.

Although these data seem to falsify Symons's (1979) hypothesis that males prefer females of high reproductive value rather than of high fertility, a cautionary note must be added. These findings are based on the inference that subtracting the preferred age difference between self and mate from the age at which one prefers to marry accurately represents the true age preferred in mates. It is possible that this inference is unwarranted, and that when males actually reach the age at which they decide to marry, they may prefer females who are younger. Nonetheless, the validity check on actual age at marriage corroborates the finding on preferred age differences between self and mates, as well as the finding that females tend to marry on average at approximately 25 years of age. Future research could profitably explore this issue in greater detail by examining mate age preferences and actual ages within both short-term and long-term mating relationships

Not specifically predicted was the finding that females prefer somewhat older mates in all 37 cultures. This finding, in conjunction with the known positive correlation between age and income among males (Jencks 1979; Willerman 1979), provides additional circumstantial evidence for the hypothesis that females prefer mates who show characteristics associated with having a high providing capacity. Older male age also could provide a cue to longevity, maturity, prowess, confidence, judgment, or experience (cf. Ellis, in press; Symons 1979). Further research is needed to uncover the functions of this crossculturally robust female preference for older males.

The fifth evolution-based prediction, that males would value chastity in potential mates more than would females, was supported in 23 out of the 37 samples. In the remaining 14 samples, no significant sex differences emerged. Samples from Africa, the Middle East, South America, and Eastern Europe generally show the predicted sex differences in preferences for chastity in a potential mate. Many of the samples indicating no sex differences were concentrated in Western Europe, Canada, New Zealand, China, and Indonesia. These results provide modest support for the evolutionary hypothesis based on paternity probability. The wide variation in preference for chastity suggests that cultural differences, ecological differences, or mating system differences exert powerful effects on the value attached to chastity.

A speculation is warranted regarding the cross-cultural variability of sex differences in chastity valuation, when contrasted with the more pervasive sex differences found 
in mate preferences for earning power, relative age, and physical attractiveness. Chastity differs from these other variables in that it is less directly observable. Even physical tests of female virginity are unreliable due to variations in the morphology of the hymen, rupture due to nonsexual causes, and deliberate alteration (Dickemann 1981). Sexual selection should favor preference mechanisms for cues that are reliably associated with characteristics that have fitness advantage for the mate selector. Where cues are not directly observable or cannot be reliably assessed, as in the case of chastity, it is difficult to imagine how specific preference mechanisms could have been fashioned by sexual selection. These considerations, of course, do not preclude selection for a more general mechanism such as sexual jealousy (Daly et al. 1982) that promotes a heightened concern about females having sexual contact with other males, either prior to or after mate choice. These speculations highlight our profound lack of knowledge about basic psychological mechanisms involved in human mating decisions (Symons 1987b).

In sum, three of the five predictions - those involving mate preferences for earning potential, relative youth, and physical attractiveness - were strongly confirmed across cultures. The prediction regarding ambition-industriousness was confirmed only in 29 samples, and showed a significant reversal among the Zulu. The chastity prediction received still less empirical support, with only 23 of the 37 samples showing significant sex differences.

\subsection{Qualifications and IImitations}

Several important qualifications must attend the in terpretation of these findings. First, the samples cannot be viewed as representative of the populations of each country; rural and less-educated individuals are underrepresented, although the samples of such individuals in this study indicate no departure from the primary predicted sex differences. Second, male and female preference distributions overlap considerably, in spite of mean differences. Third, neither earning potential nor physical appearance emerged as the highest rated or ranked characteristic for either sex, even though these characteristics showed large sex differences. Both sexes ranked the characteristics "kind-understanding" and "intelligent" higher than earning power and attractiveness in all samples, suggesting that species-typical mate preferences may be more potent than sex-linked preferences.

Other limitations surround the instruments, data sources, and operationalizations of the key constructs. Self-report contains obvious limitations and should be supplemented by alternative data sources in future studies. The close correspondence between the demographic data showing actual age at marriage data and the expressed mate preference data, however, suggests that we need not be pessimistic about the capacity of individuals to report preferences that are reflected in their actual mating decisions. Another limitation is that the single items used here may underestimate the magnitudes of the present sex differences, as they tend to be less reliable than composite clusters of items (Nunally 1978). And the set of characteristics representing each construct could be expanded to assess other mate characteristics such as the willingness of a male to invest resources, the willingness of a female to devote reproductive capacity to a given male, and behavioral cues associated with both proclivities.

A potential limitation involves the particular cultures selected for study. These samples are biased toward urbanized, cash-economy cultures. Less urbanized, noncash cultures obviously must be studied to circumvent this bias. The tremendous cultural variability with respect to chastity, however, belies the notion that these 37 samples might somehow be culturally homogeneous and gives greater credibility to the empirical sex differences that transcend this cultural diversity.

Arranged marriages in some cultures pose another potential problem. If parents and other kin arrange marriages, how could mate preferences evolve or be expressed? We lack knowledge about the prevalence of arranged marriages in our environment of evolutionary adaptedness. Nonetheless, two factors mitigate this potential problem. First, if parents do arrange the marriages of their children, there is no reason to assume that they would not express preferences reflecting the reproductive considerations on which the central hypotheses here have been based. Research on parents' preferences for the mates of their sons and daughters is needed to confirm or falsify this speculation. Second, even in societies with arranged marriages, sons and daughters do exert choice. Offspring influence their parents' choices, carry on clandestine affairs, defy their parents' wishes, make threats of various sorts, and sometimes simply elope with a preferred mate (O'Kelly \& Carney 1986). Personal preferences appear to be expressed even under socially constrained conditions.

Finally, these results yield little information about the proximate (social, psychological, physiological, ontogenetic) mechanisms directly responsible for their existence. Possible candidates include genetic differences between the sexes, sensory preferences analogous to food preferences, socialization differences during development, and structural effects at a societal level such as those that limit female access to economic resources (Buss \& Barnes 1986). Although the evolutionary hypotheses presented here are largely supported by the results, research on proximate mechanisms is needed to develop a more complete explanatory account of observed sex differences in mate preferences.

\subsection{Implications}

This is the first study to examine human mate preferences across cultures on a broad scale (cf. Kurian 1979). It exceeds prior studies in geographic, cultural, political, economic, ethnic, religious, and racial diversity. However, many questions remain unanswered. Currently unknown are the cultural and ecological causes of variation from country to country in (1) the magnitudes of obtained sex differences, and (2) the absolute levels of valuing reproductively relevant mate characteristics. The internationally consistent sex differences in mate preferences found here, however, yield insight into human reproductive history, provide hypotheses about current sexual selection, and are among the most robust psychological sex differences of any kind ever documented across cultures (cf. Maccoby \& Jacklin 1974; Willerman 1979). 
What do these results reveal about human reproductive history? They support the hypothesis that males and females have faced different constraints on reproductive success in our evolutionary past. Females appear to have been limited in reproductive success by access to resources for self and offspring. Males appear to have been limited by access to fertile females. These different selection pressures have presumably produced different male and female reproductive strategies. The greater female preference for mates displaying cues to high resource potential and the greater male preference for mates displaying cues to high reproductive capacity appear to represent adaptations to sex-differentiated reproductive constraints in our evolutionary past.

What do these results reveal about current sexual selection? No definitive answer can be provided, as we lack data on reproductive differences associated with the expression of mate preferences. The findings, however, have strong implications for human intrasexual competition - a key component of Darwin's theory of sexual selection. Mate preferences should influence intrasexual competition such that males compete with each other to display the resources that females desire in mates; females should compete with each other to display the reproductively linked cues that males desire in mates (Buss 1988a). Furthermore, mate preferences should affect opposite sex intrasexual maneuvers, such as tactics used to guard or retain mates (Buss 1988b; Flinn 1988), tactics used for mate poaching, and perhaps tactics used to derogate intrasexual competitors (Buss \& Dedden, submitted). These now established sex differences in mate preferences across 37 cultures provide a foundation for testing hypotheses about human intrasexual competition on an international scale.

Most generally, these results suggest that selective preferences in mating are not the sole province of females (Anderson 1986; Berenstain \& Wade 1983; Robinson 1982; Smuts 1987), as is implied by some evolutionary accounts that stress female choosiness. Human males and females both express preferences, and it is clear that there are powerful selective advantages for doing so. These results also implicate cultural systems in determining sex differences or the absence of sex differences. The cross-cultural variability in chastity valuation serves as a strong reminder that even mechanisms closely linked with reproduction are not "genetically determined" in the sense of being inevitable or intractable. Finally, these results support the broad hypothesis that human males and females differ in reproductive strategies, and the specific hypothesis that mate preferences represent important components of these strategies.

\section{ACKNOWLEDGMENTS}

Special thanks go to research collaborators of the International Mate Selection Project: M. Abbott, A. Angleitner, A. Asherian, A. Biaggio, A. Blanco, H-Y. Ch'u, B. Ekehammar, J. Czapinski, B. DeRaad, M. Fioravanti, J. Georgas, P. Gjerde, R. Guttman, F. Hazan, S. Iwawaki, N. Janakiramaiah, F. Khosroshahi, S. Kreitler, L. Lachenicht, M. Lee, K. Liik, S. Makim, S. Mika, M. Moadel-Shahidi, G. Moane, A. Mundy-Castle, B. Little, M. Montero, E. Nsenduluka, T. Niit, R. Pienkowski, A. PirttilaBackman, J. Ponce de Leon, J. Rousseau, M. Runco, M. Safir, C. Samuels, R. Sanitioso, B. Schweitzer, R. Serpell, N. Smid, C. Spencer, M. Tadinac, E. Todorova, K. Troland, L. Van den Brande, G. Van Heck, L. Van Langenhove, and K. S. Yang.
This article has benefited from insightful suggestions on earlier drafts by John Alcock, Richard D. Alexander, Monique Borgerhoff Mulder, Linnda Caporael, Bruce Ellis, Elizabeth Hill, Katharine Hoyenga, Carolyn Phinney, Donald Symons, Barb Smuts, Bob Smuts, Debra Umberson, and Lee Willerman. Lisa Chiodo, Lisa Dedden, and Karen Kleinsmith deserve special thanks for meticulous processing of this enormous data set. This research could not have been completed without the insight and effort of Armen Asherian in all phases of the project.

\section{Open Peer Commentary}

Commentaries submitted by the qualified professional readership of this journal will be considered for publication in a later issue as Continuing Commentary on this article. Integrative overviews and syntheses are especially encouraged.

\section{Sex, wealth, and productivity: The neo- Darwinian way}

\section{J. Barnard}

Animal Behaviour Research Group, Department of Zoology, University of Nottingham, Nottingham NG7 2RD, England

Attempts to explain human behaviour using the kinds of functional/evolutionary models routinely applied to other species have had a chequered history. For various reasons - the diversity of powerful cultural influences on behaviour, the disastrous misapplication of neo-Darwinian ideas to society for political purposes - evolutionary discussions of human behaviour are frequently accompanied by a litany of disclaimers that reflect a desire for cautious agnosticism about the role of natural selection and other vehicles of genetic change in what we do. Although many old bogeys have been laid to rest, more still lurk in the darkness for those who would build bridges across the apparent gulf between ourselves and other animals. Only a decade ago, for example, E. O. Wilson sparked a still smouldering row by adding a provocative tail-end chapter on man to his panoramic view of social evolution in animals (Wilson 1975). Despite all this, a number of well-grounded studies are providing convincing evidence for cross-cultural similarities in behaviour which fit closely with the predictions of simple evolutionary models and suggest a common evolutionary basis for fundamental characteristics of behaviour and social and sexual relationships (e.g., see reviews in Alexander 1980; Daly \& Wilson 1983; Huntingford \& Turner 1987; Wilson 1978). In some cases, these studies have important implications for our attitudes toward sensitive social issues such as child abuse, rape, and sexual stereotyping (e.g., Daly \& Wilson 1983; Lenington 1981; Shields \& Shields 1983).

Buss's analysis of human mate-choice criteria follows in this vein by comparing cross-cultural responses to questionnaires with the predictions of sexual selection and parental investment theory. Buss's article raises some interesting points about both the interpretation of apparent mating preferences and the assumptions on which his predictions are based.

The first general point concerns the analogy between the provision of limited resources and parental investment (sensu Trivers 1972) and earning capacity in human societies. The analogy assumes a connection between earning capacity and naturally selected choice criteria based on indicators of resource acquisition. Selection favours preference based on resource acquisition where the degree of acquisition determines reproductive success (e.g., by determining the availability of food 
or protection for offspring). An important question, therefore, is whether cultural analogues of resource acquisition, such as earning capacity, reflect ongoing consequences for reproductive success or preculturally selected rules of preference that no longer have a direct effect on reproductive output. Buss is not explicit on this point. Until fairly recently, anthropological opinion favoured the latter, and much of the interest of anthropologists in small-scale societies has been based on the assumption that they represent earlier points in human history when social relationships and behaviour were still subject to selection.

One of the reasons for scepticism in applying neo-Darwinian ideas to modern industrial societies is the small average number of offspring produced in such societies. Nevertheless, suggestive evidence is emerging for a role of differential reproductive success in shaping cultural traditions (Dickemann 1985). Correlations between social status and reproductive success are well known among smaller, polygynous societies like the Yanomamõ and Turkmen, but even in Western industrial societies and other societies that are nonpolygynous, wealthy, high-status individuals may enjoy greater reproductive success. A study of 400 of the wealthiest white citizens in the United States (see Dickemann 1985) revealed that they produced a greater than average number of offspring and that their children had a higher than average (99\%) rate of survival. As a result their reproductive output was increased by some $38 \%$ over the average for the white U.S. population as a whole. Nevertheless, the problem of translating this into an estimate of fitness for behavioural traits in modern societies remains acute, in part because of the effects of cultural (legal, etc.) influences on longterm (cross-generational) access to acquired resources (Dickemann 1985; see also Vining: "Social Versus Reproductive Success" BBS 9(1) 1986).

Buss's predictions regarding sex differences in preference for physical attributes and earning capacity, as well as for other characteristics (ambitiousness, industriousness) indicating potential to acquire resources, are based on the standard assumption that females as the more heavily investing sex, will be more discriminating in selecting mate quality. Whereas this general prediction is uncontroversial, some of the specific assumptions and predictions about indices of quality are more open to question. The prediction that males should prefer younger mates compared with females, for instance, is based on the assumption that female reproductive value is more closely agedependent than that of males. This is likely to be true, but other factors (e.g., the tendency for protracted paternal care and the chance to produce attractive sons, which may increase a female's long-term reproductive success through multiple mating) also make youth and physical appearance plausible a priori criteria for female mate preferences. Furthermore, older males who are still apparently unpaired might arouse suspicions of low fidelity and low paternal investment.

Although it is worthwhile to seek robust cross-cultural sex differences in mate preferences, and cultural factors such as arranged marriages and noncash economies can probably be regarded as noise, it would be useful to control for demographic features that are likely to bear directly on preference through their effects on mate availability. Sex ratios and mating systems are the most obvious and easily incorporated. Data from some Western industrialized societies show a gradual shift in the sex ratio in marriageable age groups away from a longstanding preponderance of females toward a marginal male bias (OPCS 1988), a shift brought about through the effects of prolonged peace and reduced infant mortality on male survivorship. There is evidence suggesting that the consequences of this steadily changing sex ratio for mate competition are already having effects on attitudes toward physical appearance and marriage within sexes (e.g., Jowell \& Witherspoon 1987). As the relative earning potential of the sexes is also changing in some societies, the effects of this on male mate preferences - especially in relation to age - may be interesting. Buss's tentative explanations for male age preferences coming out at around the estimated peak of female fertility hinge on potential characteristics (such as compatibility and maturity) that are more applicable to long-term pair bonds than to the short-term matings predicted by a simple evolutionary model. Cultural changes affecting relative sex roles in resource acquisition may affect these predictions, however.

The evolutionary predictions tested by Buss are very coarsegrained. A little refinement might yield some more precise and powerful fits with predictions of a neo-Darwinian model. Given the variability within Buss's sex differences, cross-cultural comparisons based on measures of relative sex roles and mate availability could be informative.

\section{Diversity: A historical/comparative perspective}

\section{Ray H. Bixler}

Department of Psychology, University of Louisville, Louisville, KY 40292

Evolutionary behavioral science (EBS) deals with questions of little concern to most psychologists and social scientists, who for ideological or scientific reasons reject the concepts and methods that we evolutionists explore and use. Our methods are "unscientific" and our concepts steeped in "poorly disguised Social Darwinism." We have handled the latter much more comfortably than the charge that we are unscientific.

Our difficulties may stem in large part from our history; most of us (many biologists excluded) have never been exposed to systematic training in neo-Darwinian theory. We are scientists who, trained to account for behavior as a function of learning, culture, parent-child relations, and other environmental determinants, sometime later discovered and embraced a "new" frame of reference. Naturally, we have tended to retain the subjects, methods, and concepts traditionally used in our respective disciplines. As a result, our research and theory leave much to be desired.

Our haphazard training in natural selection theory and the scorn of our experimental colleagues, especially in psychology have probably served to retard us in developing and using essential evolutionary concepts and methods. EBS methodology has a long and respectable, if neglected, history (Darwin 1859a; Gould 1986; Mayr 1982; 1983; Simpson 1964). Under the subheading "Experiment versus Comparison," Mayr (1982) explains:

Each science demands its own appropriate methods. . . The alternate to experiment, clearly, is observation. . . . Mere observation, however, is not sufficient. . . A method ... which is peculiarly suitable for the study of diversity is the comparative method. . . . It is important to emphasize the scientific legitimacy of the observationalcomparative method because the experimental method is inapplicable to many scientific problems. ... Observation in biology has probably produced more insights than all experiments combined. (pp. 30-32)

Appropriate EBS methodology involves the systematic observation of behavior throughout the life span of a reasonable sample of both sexes of each of a number of related and unrelated species in varying natural environments. This method allows one to draw inferences about why the behavior of species, the two sexes, ages, and so forth, evolved-providing, of course, that we find evidence that the behaviors are, or were at one time, likely to produce an advantage in reproductive competition.

Obviously, no single investigation can achieve these goals, but the extent to which any research contributes to our understanding of natural selection will be related to how well and to what degree the methodology is used. Buss's research meets the criterion of being a reasonable sample of both sexes in varying 
natural environments. His method involves a very brief systematic observation of a behavior (questionnaire responses) unique to our species, making species comparison difficult. Nevertheless, cross-species analysis of a sort could be achieved by comparing, wherever possible, humans' statements regarding their behavior with the actual behavior of other species. No systematic effort was made to do this. No attempt to relate the questionnaire responses to reproductive success was made.

Buss cites several of these limitations and is most cautious in his conclusions. It seems to me that he has generated several meaningful hypotheses about the role of natural selection in human mating strategies which could be tested using the historical/comparative method. Comparison requires finding infrahuman equivalents of such concepts as "good financial prospect."

There are a number of other points worth mentioning:

1. The American results reported by Buss and Barnes (1986) demonstrate that both sexes find other characteristics more important than the sex differences Buss cites in his study. "Kind and understanding" was a clear preference for both sexes. (Who benefits from such a partner?) Women preferred eight characteristics, including "physically attractive" and "healthy," over "good earning capacity," and males preferred three traits over "physically attractive." One can only speculate how these various characteristics might interact in real life to produce mate preference and how various constellations are related to reproductive success.

2. Comparative analyses clearly support the male's preference for mature (proven) female mates, and the female's preference for supportive (kind and understanding?) and/or strong and dominant males (Anderson 1986; Berenstain \& Wade 1983; Goodall 1986; Hrdy 1981; de Waal 1982).

3. Perhaps establishing the statistical significance of sex differences in the professed preference for each characteristic in a list is an improper use of an excellent tool. Buss points out what many seem to forget: There is a wide range of individual differences and marked overlap between the sexes. If we add the observations made in the first point above and the probability that we respond to available potential mates as intact phenotypes rather than as a composite of a list of characteristics, the statistical significance of sex differences in preferences for "good looks" and "ambition," for example, may be largely irrelevant if not misleading predictors of reproductive success. Mayr's remarks about mathematics and "intentions" (1982, pp. $39-42,51)$ and about traits $(1983$, p. 327) may increase our ability to cope with these issues.

4. In our eagerness to find differences in behavior that we could correlate with reproductive success, we have greatly oversimplified the magnitude of sex differences. Hrdy (1981) has demonstrated that females compete; she concluded that it was quite naive to think that natural selection would not apply to them as well as to males. Buss now points to evidence that selective pressures are not the sole province of females. Unfortunately, there will be those who fail to recognize that "good looks" in a mate are very important to many women (see also Cunningham 1988;Freedman 1979) and that "industriousness" in a potential wife is crucial to many males.

\section{Typology and human mating preferences}

\section{Gerald Borgia}

Department of Zoology, University of Maryland, College Park, MD 20742

Buss is interested in testing hypotheses about the evolutionary basis for mate choice in humans. Most recent comparative animal and human studies in evolutionary biology have focused on between-group differences and tests of predicted relationships among variable traits. Buss has focused on similarity between groups, testing the hypothesis that there are species- typical patterns of mate preference. He suggests that the evolutionary process has been sufficiently consistent over time to produce a pattern of choice that is, at least partly, culturally universal. Traits such as male preference for beautiful mates are predicted to be consistent across cultures, because historically these traits have been consistently associated with reproductive success. Buss then attempts to test this hypothesis with the prediction that he will find constant patterns of sex-related differences between mating preferences in different cultures.

Buss's analysis has several important difficulties. First, he implies a typology for "evolutionary" hypotheses about social behavior that does not exist. This results because his predictions assume certain facts about human societies that may not always be true. For example, the prediction of an asymmetry in the importance of preferences based on earning potential and ambition-industriousness assumes greater male access to resources. Although male domination of resources may occur in many societies, it is not always present, and the "evolutionary" prediction should not apply to these exceptions. Thus, predictions for particular behavioral traits, such as mating preference, are often complex and depend on the particular pattern of social arrangements existing in a society. Buss's typological analysis does not allow for these exceptions within the framework of an evolutionary hypothesis.

A common but incorrect criticism of evolutionary studies of human behavior is that they are typological in exactly this sense. Exceptions to general patterns are cited as refutations of the entire approach. The correct answer is that this is not true, because evolutionary biologists recognize that expected patterns often depend on special social contexts. Buss fails to give this important point its due.

Second, the analysis as presented is flawed because the author doesn't use a valid sample. Of the 32 societies in his comparisons, 27 are European or have had a predominantly European influence. Aboriginal cultures are so scarce in the sample that the geographical category "Oceania" is represented only by the transplanted British cultures in Australia and New Zealand. Long ago, anthropologists recognized the role of cultural transfer in creating between-group similarity in social traits. Murdock and many more recent workers have gone to great lengths to exclude the effects of cultural transfer as a cause of similarity. Buss's use of a sample with an overwhelmingly European bias and no significant variation in cultural traits that are likely to be associated with different patterns of mate preference (e.g., with variation in mating system, residence, or inheritance patterns) does not present a reasonable challenge to the hypotheses he claims to test. Buss claims as evidence for the independence of his sample the fact that in one variable, the preference for female chastity, there was some variation in response among the groups he sampled and that this differs from the patterns seen for other variables. This is not a compelling argument, however. There are many potential causes for variation in a sample apart from statistical independence. For example, the variance in the sampling practices he used could certainly account for the variation in preference for chastity. Moreover, even for this variable, most males in the social groups he sampled showed the same preference. Thus, Buss has failed to do what is necessary in this type of comparison: offer convincing evidence that the observed similarity in cross-cultural patterns of mate preference is due to convergent evolution and not to cultural transfer.

Third, there is an important inconsistency between the conclusions of this study and the history of the societies use in these tests. The majority of societies in Buss's sample are drawn from industrialized countries in which there has been a dramatic change in key reproductive characteristics (commonly referred to as the demographic transition), including changes in fecundity and age of first reproduction. The occurrence of the demographic transition contradicts Buss's suggestion that basic human reproductive patterns are invariant across cultures. It suggests that the consistency in patterns of preference found by 
Buss is due to the homogeneity of his sample, rather than to the constancy of female reproductive patterns.

Buss claims that "this is the first study to examine human mate preferences across cultures on a broad scale." This statement ignores a large body of cross-cultural comparisons on mating preferences collected by anthropologists and evolutionary biologists (e.g. Flinn 1981). Perhaps if Buss were more familiar with that work, he could have avoided the obvious errors in his own analysis.

\section{Mechanisms matter: The difference between sociobiology and evolutionary psychology}

\section{Linnda R. Caporael \\ Department of Science and Technology Studies, Rensselaer Polytechnic Institute, Troy, NY 12180}

I share Buss's objective of an integration between psychology ${ }^{\mathrm{I}}$ and evolution, but the synthesis cannot be - nor need it be grounded in sociobiology. Any proposed solution to the longstanding impasse between advocates of "mostly cultural" versus "mostly biological" explanations for behavior will inevitably be evaluated by the traditional standards of the social and behavioral sciences for methodological rigor and explanatory validity.

Sociobiologists are likely to judge Buss's research to be a strong demonstration of sex differences. From a fairly simple model founded on genetic self-interest as the ultimate causal factor in behavior, he has derived a set of hypotheses about human preferences. The predictions are supported across 37 samples. The proximate mechanisms are unknown, but his findings of cross-cultural universality argue compellingly for biological factors and provide the grounds for inferring the differences in constraints faced by males and females during their evolutionary history.

Psychologists will find the target article problematic, largely because the traditional "rules of the game," from hypotheses to conclusions, are contravened to no apparent purpose. The evolutionary hypotheses do not describe some heretofore unnoticed but significant behavior, nor are they contrasted with nonevolutionary hypotheses. The same predictions could have been made from a random sample of newspaper advertisements, magazines, or soap operas. At the very least, the evolutionary framework could have been used heuristically to suggest novel comparisons. For example, is there a relationship between women's preferences for men who are good financial prospects and the level of state-supported maternity and childcare benefits? Nevertheless, this heuristic use would still leave open serious doubts as to the ultimate disconfirmability of the sociobiological framework (Kitcher 1985).

The data analysis also deviates from generally accepted research practice in psychology. It exploits the well-known fact that very large sample sizes produce statistically significant results even when the differences between groups are very small. Without an analysis of the magnitude or power of the effects, we have no way of distinguishing an important result from a trivial, albeit statistically significant, one. Furthermore, most psychologists will have serious reservations about the appropriateness of using a large number of $t$-tests.

What Buss is saying has evolved is also a problem. In most of his target article, he suggests he is studying preferences, which are psychological mechanisms. But toward the end, he says his work yields no information about proximate mechanisms which may be preferences, genetic differences, socialization effects, or even "structural effects at a societal level such as those that limit female access to economic resources." But how can structural effects at a societal level be accommodated in a framework based on biological selection for heritable traits? Without a clue to the mechanism, there is no way to distinguish evolved sex differences from the multitude of gender differences that have no basis in biological evolutionary processes.

For example, consider an alternative account of the relative preferences males and females show for financial prospects in a mate. Both sexes may want the same financial resources, but because women are systematically denied independent access to them, we may conclude that (a) women select the most practical remaining option - marriage to men who have the resources, and (b) men do not use irrelevant criteria for their mate preferences. This explanation suggests that there are no evolved sex differences: Males and females have identical preferences, but social structural arrangements produce gender differences. By itself, evolutionary theory cannot explain why the social structural arrangements exist, nor do the social structural arrangements reveal anything about evolutionary history (cf. Buss \& Barnes 1986). An understanding of proximate mechanisms cannot be postponed, as Buss suggests, to a later date; we must understand the mechanism before we can determine whether it has an ultimate cause at all.

The emphasis on mechanism is a key distinction between sociobiology and what could be dubbed evolutionary psychology. ${ }^{2}$ At least in the human case, there are two requirements for an evolutionary argument. The first is an account of the origins of the hypothesized mechanism given the species morphology and ecology in which the mechanism is presumed to evolve; the second is an account of the systematic engagement of the mechanism under a variety of social conditions. An evolutionary psychology would draw more from a functionalist paleoanthropology (Foley 1987) than from evolutionary theory per se. In contrast to the sociobiological strategy, which tends to provide evolutionary accounts of contemporary phenomena, research questions in evolutionary psychology would be developed from engineering design considerations. Given the specifications of morphology and ecology, what are the minimal functional requirements for reproduction and development to reproductive age? What possible mechanisms might we expect to evolve under those conditions? How would these mechanisms behave under changing historical, structural, and cultural conditions, such as during the shift from living in small groups to high residential density, from kinship loyalty to state loyalty, and from rare interactions with strangers to daily interactions in large numbers? What research designs allow us to choose among a variety of possible evolved mechanisms (which would allow us to elaborate the evolutionary scenario)? What research designs allow us to eliminate alternative explanations or show how the purportedly evolved mechanism interacts with historical and contemporary social structures? I suspect that the mechanisms that we conclude have evolved will be relatively "low-level" that is, they will have to do with such things as attentional biases, maturational sequences, or rules for evaluating and integrating different categories of information. An evolutionary psychology will be most successful when it makes novel predictions, organizes previously inexplicable research results, and suggests novel comparisons.

In the final analysis, the results reported by Buss are too compelling. Consider a thought experiment. Suppose that the pattern of results were reversed; for example, that men were found to prefer women who were good financial prospects and that women were found to prefer men who were young and attractive. Most observers would probably conclude that the measuring instrument was seriously flawed. What then is the point of scientific investigation if only one pattern of results can be accepted as valid? Science has helped demythologize some purported biological sex differences (Maccoby \& Jacklin 1974), but it has also facilitated the social construction of other differences supposedly based on biological imperatives (Harding \& O'Barr 1987; Pfafflin 1984. [See also McGlone: "Sex Differences in Human Brain Asymmetry's BBS 3(2) 1980.] In a society that feels a stronger obligation to correct inequities arising from imperfections in the social system than to correct those resulting from differences in natural endowments (Lambert 1987), the 
attribution of evolved sex differences takes on new meaning: Why should the resources of a society be used to provide women with good financial prospects of their own when their "natural" preference is for men with such prospects?

\section{NOTES}

1. For this commentary, I am using the term psychology to refer to social, cognitive, and developmental psychology.

2. William James forged an evolutionary psychology before the advent of behaviorism. Although some of his concepts and conclusions are outdated by contemporary psychological research, the approach he used is similar to the one I sketch here. A contemporary illustration can be found in Caporael et al., submitted.

\section{Sex differences in life histories: The role of sexual selection and mate choice}

\section{Charles Crawford}

\section{Department of Psychology, Simon Fraser University, Burnaby, B.C.,} Canada V5A 156

The evolution of a genetically organized life history is governed by the way resources are allocated to survival, growth, and reproduction at each age throughout the lifetime of a typical individual (Wittenberger 1981). Tactics consist of decision rules and procedures for implementing the life history (e.g., if you are male and small, attempt sneak copulations; if large seek mating territory). We can think of human males and females as individuals using different tactics to pursue somewhat different genetic life histories. However, the fact that each individual must have one parent of each sex, and hence that males and females have equal reproductive value (Fisher 1930), constrains the divergence of male and female life history traits in species with a 1:1 sex ratio.

During human evolution, males and females may have inhabited slightly different ecological niches, with males spending more time hunting and warring and females spending more time gathering and caregiving. The result could have been sex differences in the way males and females allocate resources to survival, growth, and reproduction. Human physiologists often regard sexual division of labor as the evolutionary source of human sexual dimorphism. For example, Kimura (1987) recently suggested that sex differences in brain anatomy are related to sex-role division of labor. Another explanation for sex differences is sexual selection - the members of the low-investment sex (usually males) compete with each other in the elaboration of features giving them access to the high-investment sex (usually females; Trivers 1985). It is likely that both types of selection acted jointly on our ancestors to produce the observed human sexual dimorphism. However, in his research, Buss concentrates primarily on predictions made from parental investment and sexual selection theory.

It is not easy to test hypotheses about the evolutionary significance of human behavior: the conditions in the ancestral environment that rendered certain traits adaptive and others nonadaptive, and the effects on current behavior of mechanisms that evolved to produce those ancestral behaviors. The absence of extant Homo species, other than sapiens, makes historical and comparative analysis difficult. Measures of current reproductive success are an unreliable guide because recent environmental and cultural changes may make formerly adaptive behaviors nonadaptive or even maladaptive. We must therefore put considerable emphasis on using contemporary data to test hypotheses generated from assumptions about ancestral conditions and behavior.

To make strong statements about the evolutionary significance of human behavior, one must do at least three things (Crawford \& Anderson 1988): (1) use contemporary data to test predictions derived from scenarios about ancestral behaviors,
(2) develop quantitative models to determine whether a behavior could have contributed to fitness in an ancestral population, and (3) study proximate psychological mechanisms associated with the behavior, that is, Darwinian algorithms (Cosmides \& Tooby 1987). No single piece of research can meet all three criteria. Buss's work focuses on the first.

Stronger inferences can be made if predictions from more than one evolutionary explanation are tested in conjunction with at least one nonevolutionary hypothesis. Buss is to be commended for comparing two evolutionary predictions about male preferences, that is, for fertility and reproductive value. His results appear to support fertility. However, the age of puberty differs considerably across cultures. Reproductive value also differs between cultures, although not as much as age of menarche. I would be interested in differences on these two variables for Buss's 37 samples.

Nonevolutionary predictions are those that do not make explicit reference to fitness in ancestral population. There are implicit, nonevolutionary predictions in Buss's work, for example, that males prefer wealthy females because of the resources they provide. I believe that stronger statements could have been made if competing evolutionary and nonevolutionary predictions had been more systematically presented.

A strong demonstration of sexual selection in humans requires one to show (1) that there is really a preference for particular attributes in a mate, (2) that there was a fitness differential associated with this preference in ancestral human populations, and (3) that the fitness differential is not more parsimoniously explained by ordinary selection for survival and reproduction. Buss's data provide some impressive evidence for differences in male and female preferences. However, the case would have been strengthened if some data from more traditional societies, such as the !Kung, had been included.

Showing that a fitness differential existed in ancestral human populations is not a simple task. One approach would be to construct a quantitative model of how the behavior might have contributed to fitness in an ancestral population and to apply it to data from current hunter-gatherers (Anderson \& Crawford 1988) or to use it in computer simulations of ancestral population processes.

Finally, distinguishing the role of ordinary and sexual selection is not a straightforward matter. For example, males may prefer physical vigor in females. However, female vigor could have been selected because it contributed to ancestral females ability to produce and rear offspring, and may have little to do with male preferences.

Clearly, human males and females have slightly different life histories. Males are larger, mature later, die at a greater rate at all ages, are capable of reproducing into old age, and so forth (Trivers 1985). Differences in male and female mate preferences provide one of the windows on human nature. Buss's study of mate preferences in 37 samples from around the world provides a good view through that window. We must continue looking through it.

\section{Aggregates, averages, and behavioral plasticity}

\section{Mildred Dickemann}

Department of Anthropology, Sonoma State University, Rohnert Park, CA 94928

In the long, slow climb of the social sciences upward from the racism and ethnocentrism of the nineteenth century, psychology has been the tardiest sibling, perhaps because it has been only grudgingly "social." Those comparative approaches that have been the best cure for narrow-minded theory and method have had only a fitful life in this discipline, which has been 
characterized by a sequence of theories of "human nature," each generalized from a single race, class, and time in Western society. Buss's study is perhaps a step in the right direction, and may provide the largest cross-societal sample of mating "preference" ever; but for an anthropologist, it has many problematic aspects.

What is the posited relation between evoked "preferences" (wishes, desires) and behavior? Is preference assumed to be a better measure of human evolutionary nature than action? Apparently not, as in one case (age at marriage) behavior is used as a "validity check" on statements of preference. Does this mean that unconsummated preferences (like my wish for wealth) are evolutionarily meaningless? Yet the assumption that preferences have evolutionary meaning is explicit. Are they less plastic, less socially variable than actions? Buss states that the results "yield no information about the proximate . . mechanisms," but is not preference proximate to action? What, then, is the rationale for a study of preference that largely ignores the vast bodies of existing data on human mating behavior?

The neglect of relevant literatures is reflected in several aspects of method and analysis. Why, for example, are "ambition" and "industriousness" treated as a single attribute? The ethnographic literature would reveal their distinctiveness: Industriousness is prized in women not only among the Zulu but wherever women's work has value, whereas ambition is generally seen by husbands and parents as threatening to the marital hierarchy. Several attributes ("good financial prospect," "earning capacity") are meaningless in groups not primarily engaged in wage labor: How were these translated into meaningful attributes for peasant and subsistence societies? Besides food, shelter, territory, and protection, humans seek other mate attributes, such as social rank or socioeconomic status, size of kin or alliance network, political power, responsibility, honesty, and family history of physical and mental health. Were these included? What is "good looks"? Does it include fat deposition, physical strength, height, or attributes of the dominant racial group such as blondness? How are we to interpret the results without knowledge of the contents of these terms?

Buss recognizes the biased nature of the sample "toward urbanized cash-economy cultures," but provides no justification for its selection. More than cash economy is involved: At least 25 of the 37 samples have completed the demographic (fertility) transition, and several are in mid-process; perhaps 8 have completed the "second demographic transition" (zero to negative growth rate). This is clearly reflected in the results - for example, those in regard to "chastity" - but nowhere is the sample disaggregated in these terms. Buss states that the results in regard to "chastity" "provide modest support for the evolutionary hypothesis based on paternity probability." Apparently there is more than one such hypothesis, for they provide strong support for my own version (Dickemann 1981, cited by Buss), which emphasizes that the value placed on female virginity/fidelity varies, depending upon the relative degree of male investment (Dickemann 1979a; 1979b; 1981; see also Daly \& Wilson 1983 and Flinn 1988, both cited by Buss).

"We lack knowledge about the prevalence of arranged marriages in our environment of evolutionary adaptedness" writes Buss, ignoring vast historical, sociological, demographic, and anthropological literatures on this subject. (For Western Europe alone, some major works are Duby 1978; Goody 1983 Segalen 1986; Stone 1977; for anthropology one might note Lévi-Strauss 1969; Murdock 1949; Whyte 1978.) These would reveal that the degree of children's contribution to mate choice varies from null (prenatal and infant betrothal!) to veto power, to fully independent choice (after parental socialization); that violations of parental decisions may be punished by exile or death; that consensual unions are by no means limited to Sweden; that there are times and places in which older-female marriages are socially and statistically significant (including the Tiwi; see Hart \& Pilling 1960, as cited by Buss); and that there are historical, demographic, and anthropological theories to explain these variations, mostly involving the structure of the marriage market, the intensity of mate competition, and the association of prospective spouse with resources or powerful kin and allies (in addition to my own work cited above, see Flinn \& Low 1986; Irons 1983).

The existence of this diversity, juxtaposed to the study before us, reveals the latter's most fundamental theoretical flaw: the assumption that human reproductive behavior is a set of invariant responses arising from some set of invariant evolutionary dicta. Yet everything we know about human biology and social behavior tells us that our "adaptedness" consists of a capacity to grade and modify responses in relation to socioenvironmental circumstances, a highly evolved phenotypic plasticity that is central to Darwinian behavioral biology, human and other, and clearly explicated in several sources cited by Buss, including my own work (see especially Bateson 1983; Borgerhoff \& Mulder 1988; Daly \& Wilson 1983). Immerson in the relevant social science literatures, prior to articulation and operationalization of the hypotheses, would have assisted in the elimination of the ethnocentrism evident here, and in the design of protocols reflecting human plasticity. The use of randomly chosen samples to produce aggregate averages obscures rather than reveals this adaptive plasticity. Washing away differences in economy, marriage system, social class, urbanization, and ethnicity with larger and larger aggregates takes us further away from, rather than closer to, the nature of human nature. It is hard to see what has been gained by the time, energy, risk and, no doubt, money spent on this project. It is time for psychologists to develop the suspicion of aggregate data that now pervades responsible demography, history, and anthropology, and attend to human diversity and the increasingly well-understood reasons for it. Otherwise, psychological projects will continue to suffer from the insularity that has characterized so many of them in the past.

\section{Spouse preference shifts with age}

\section{Susan M. Essock}

Office of the Commissioner, Connecticut Department of Mental Health, 90 Washington St., Hartford, CT 06106

Buss and his colleagues have gathered a valuable data set and offered fresh support to the broad hypothesis that human males and females tend to differ in their reproductive strategies and that differences in mate preferences play a role in these differences in reproductive strategies. A great strength of the database is that a similar instrument was used across the diverse groups sampled. This is an enormous improvement over the use of a much more highly derived ethnographic atlas (e.g., Murdock 1967), with its accompanying introduction of uncertainty and bias. At last, here are analyses of broadly cross-cultural data that are much better suited to quantification and that are not derived from interpretations of interpreters' interpretations of norms (two weaknesses of an ethnographic atlas).

Buss's data and the data from an ethnographic atlas can both be criticized as collections derived from biased, ill-specified sampling techniques that often rely on reported, rather than observed, behavior. I do not agree with Buss that "the wide variety of sampling techniques used tends to increase the generality of consistent results that do emerge by minimizing the biasing effects of any particular sampling procedure," that is, that many biased samples are unlikely to merge to create an unbiased sample. But, even granted the design limitations, Buss's data set represents a large improvement over its most notable predecessors. And, although investigators must be cautious about interpreting repeated examinations of the same data as though each look were a new, independent sample, these data bear further looking. 
It would be interesting to go on to examine, for males and for females, how their preferred age for spouses varies with their own ages. If the conjectures put forth by Buss and others about mate selection as a fitness-optimizing activity are true, then adult males of all ages should continue to prefer reproductiveage females (i.e., older males should prefer greater age differences between themselves and their spouses than should younger males). In contrast, for females, a subject's age would not be expected to be a significant covariant for the preferred age difference between self and spouse. Rather, reproductiveand postreproductive-age females would be expected to differ in the relative weight they place on some of the characteristics of potential spouses (e.g., their heritable traits versus their reliability as reciprocation partners) - traits that are largely independent of the age of the potential spouse.

The means and standard deviations given in Buss's Table $\mathbf{l}$ for the subjects' ages suggest wide variation in the age distribution in many of the groups sampled (e.g., India). Buss and others asking similar questions could further enrich their analyses by studying how preferences vary with age.

\section{Darwinism versus neo-Darwinism in the study of human mate preferences}

\section{Michael T. Ghiselin \\ California Academy of Sciences, Golden Gate Park, San Francisco, CA 94118}

The works of Charles Darwin are often cited, sometimes read, and occasionally understood. How well we understand them is of course a matter of degree. If we, perhaps rightly, ignore peripheral issues, we should nonetheless try not to miss the fundamental principles and the most interesting questions. With respect to the theory of sexual selection expounded on the pages of The Descent of Man, misunderstandings have become so insinuated into academic tradition as to masquerade as fact.

We continue to be told that sexual selection was an ad hoc hypothesis, intended to explain away facts that could not be explained in terms of natural selection. Actually it was an experimentum crucis, providing a compelling argument for selection theory in general (Ghiselin 1969; see also Ghiselin 1974; West-Eberhard 1983). The three modes of selection artificial, natural, and sexual - have quite different effects. Sexual selection is pure reproductive competition, and depends upon efforts to monopolize sexual reproduction as such; therefore, it can lead to the evolution of adaptively neutral or even maladaptive features. Such competing hypotheses as "special creation," orthogenesis, and sundry versions of Lamarckianism generate no such predictions.

The term "neo-Darwinism" was coined by Darwin's supporter George John Romanes to designate certain alternatives to "Darwinism" (see Romanes 1893-97). Perhaps the best definition of "Darwinism" is the following: "Natural Selection has been the main but not exclusive means of modification" (Darwin 1859 , p. 6). Other mechanisms in Darwin's theory included use and disuse, what are now called pleiotropy and developmental constraint, and of course sexual selection. Darwin himself was not quite sure how important these various mechanisms were, but he clearly did invoke them. "Neo-Darwinism" may be defined as the belief that natural selection is the exclusive cause of modification. A strict version of neo-Darwinism is obviously untenable, so it usually has to be qualified "with trivial exceptions." For the first historically important advocate of neoDarwinism, Alfred Russel Wallace, the trivial exceptions were such things as the human intellect (see Wallace 1870).

Neo-Darwinists have generally advocated Panglossian adaptationism, and Wallace's opposition to sexual selection was symptomatic of this. The other important early neo-Darwinist, August Weismann, tried to explain all sorts of things (e.g., sex and death) as consequences of species advantages of the sort that Darwin and present-day mainstream evolutionists have rejected out of hand (see Weismann 1891-92).

Neo-Darwinists have various ways of dealing with mechanisms other than natural selection. They can admit that these exist, but trivialize them, and this has often been done with respect to genetic drift and other forms of sampling error. Another maneuver is to conceal their existence, and this is often done by asserting that all selection is "really" natural selection. I will not belabor the disadvantages of dividing selection into natural natural selection, sexual natural selection, and artificial natural selection. Another possibility has been to claim that the facts explained by sexual selection are really the consequence of natural selection. A popular thesis has been that sexual "ornaments" are really species-recognition mechanisms and the like. More important for the present discussion is the claim that sexual selection is really a form of natural eugenics. Although this notion goes back at least as far as Erasmus Darwin (1794, p. 503), R. A. Fisher (1930) gets credit (or blame) for making it a part of neo-Darwinian orthodoxy. Such notions tend not to be tested against experiential data, but are merely presupposed. When they are examined critically, they do not hold up very well (Eberhard 1985).

That organisms evaluate resources is well supported by the data of experience. Mates obviously differ with respect to their ability to provide support for offspring, and it stands to reason that they will be evaluated as such. But this is not sexual selection. It is not a form of pure reproductive competition. The same is true when the resources in question are genetic ones. So most of the choice that Buss discusses has little, if anything, to do with sexual selection (see also Parker 1987). But how does sexual selection enter into mate choice, if it does enter into it at all?

This is hardly the place to develop even a sketch of a Darwinian, in contradistinction to a neo-Darwinian, theory of human mate choice. But it does seem worthwhile to suggest what form such a theory might take, for it probably would help to interpret Buss's data. Sexual selection by male combat has probably been a major reason for male humans marrying somewhat later than female ones. In many species, the males are perfectly fertile for some time before they invest maximally in efforts to monopolize females. Rather, they reproduce opportunistically, and avoid combat, when young.

Human beings function as hermaphrodites, in the sense that both sexes provide resources for their offspring. Therefore, the possibility exists for sexual selection by male choice as well as by female choice. One would therefore expect the evolution of hypertelic secondary sexual characters to evolve in both sexes. Features that are attractive to members of the opposite sex, for whatever reason, would tend to be accentuated. Sometimes these may be just the differences that distinguish the two sexes. If early proclivity to marry were characteristic of females, then sexual selection might favor women who appear younger than they really are. Nonetheless, the differences between the sexes depend on a process of growth and maturation over a period of several years. It is possible that human males mate preferentially with those females who are most stimulating erotically, in spite of the genetical arithmetic. If this is so, then Darwin was right, and Fisher was wrong. Buss's finding that human males prefer mates who are past the age of maximal reproductive value tends to support the Darwinian interpretation. 


\section{Missing link in mate preference studies: Reproduction}

\section{Brian A. Gladue}

Program in Human Sexuality, Department of Psychology, North Dakota State University, Fargo, ND 58105

Too often, the mysteries and subtleties of human mate selection and attraction get oversimplified or otherwise converted into grand biological entities. Most studies attempting to understand human behavioral evolution, especially the reproductive history of the species, look to mate selection and mate preference as indicators of underlying behavioral tendencies and evolutionary trends. There are guesses made as to what transpired over the millenia of human behavioral evolution. Ultimately, numerous facets of current male-female attraction and mate selection are described, and some connections between these findings and our species' ancestral behavioral origins are assumed. And for the most part, most of these studies' findings and interpretations, at first glance, seem sensible and concur with ordinary experiences of scientists and laymen: Females prefer older, "ambitious and industrious" males possessing "good financial prospects." Males, more than females, prefer someone younger with "good looks." The various predictions in the target article and the accompanying data seem to support the central theme that all of these aspects of mate selection are indicative of an underlying historical biological trend toward maximizing reproductive success.

Yet, there is a missing piece in this seemingly complete picture: reproduction. As any sociobiologist knows, the bottom line for "success" in biological terms is measured in terms of the number of surviving offspring people have throughout lifetimes. Monitoring aspects of mate selection without considering reproductive attitudes or behaviors associated with those mate preferences can be very misleading. In fact, the omission of reproduction as a key measure in any study of mate selection reduces that study to one of "dating" or simply "companion/sex partner" preferences. One cannot merely assume that a desire to seek out a person possessing the right mix of financial and beauty factors translates into a desire to produce offspring with that person. Of course, determining reproductive success and fitness in a prospective mate is tricky business. One theoretician suggested that we should ask prospective mates to produce records of their grandchildren as evidence of mating desirability (Whalen 1980). In real time, however, people do make reproductive choices based on factors other than appearance and income.

Consider the telling fact that the measure with the greatest variation (and thus disagreeing most with Buss's set of predictions) is the one closest to reproduction: previous experience in sexual intercourse. Although most humans across the globe seem to agree about financial prospects and physical beauty (however defined) in a "mate," the agreement breaks down when it comes to sexual experience. In addition to this simple measure of reproductive history, it would have been extremely helpful to have data on attitudes toward family size, desired number of sex partners before (and even during) marriage, reproductive health, and other items in order to obtain a general picture of desired reproductive history in both the rater and a preferred mate. Instead, according to Buss, the variability in the sexual intercourse data "suggests that cultural differences, ecological differences, or mating system differences exert powerful effects on the value attached to chastity." One of these days, sociobiological theorists will not use cultural or mating system differences to excuse findings they don't like, while ignoring those same cultural differences as explanations for findings they do like. The schema and model must be consistent. If bioevolutionary explanations don't fit nicely with the chastity data, why should they account for the findings regarding "good financial prospects" or "ambition and industriousness"? Buss can't have it both ways.

There is another, real-time, explanation for these findings. Let us, like Buss, assume a background of basic human evolution toward gender differences in preferred mate characteristics. Let us then consider how current differences in technological development and political/religious philosophy might account for variations from this base. For example, Buss has data broken down by countries, yet he assumes that they are all different cultures, when in fact these data show cross-country, yet ethnic, similarities (the Scandinavian data are a good example, where Norway, Sweden, and Finland are nearly identical on the chastity measure). Also, considering the data not so much by country but by predominant religion may tell more about human diversity than mere geography. One could safely wager that Muslims, Christians, animists, and atheists may differ on various reproductive themes, attitudes, and behaviors. Elsewhere, the "financial prospects" data could be analyzed by gross national product rather than by country, or by categories of developed versus developing sovereignty, because desired family size actually decreases with increasing per-capita income in developing countries.

Finally, questions not asked (but perhaps included in future surveys) could tell us much about desires not only for marriage partners but also for reproductive partners: What about issues of family size, birth control use, and sexually transmitted disease? And what about supposedly "valuable" parental, investmentmale traits such as territoriality and aggression? Do females now favor these in a prospective mate, even if they could be considered counterproductive (e.g., correlating with increases in child/spouse abuse, accidental death, and family abandonment)? Despite good intentions to assume that mate selection based on finances, age, attractiveness, and chastity somehow includes all this, we will never really know until we ask. Certainly Buss has taken a major step toward beginning this endeavor. But let's not infer basic human reproductive strategies from simple features of desired-companion attitudes and beliefs.

\section{Intersocietal variation in the mate preferences of males and females}

\section{Norval D. Glenn \\ Department of Sociology, University of Texas, Austin, $T \times 78712$}

Buss's target article is an important contribution, and one for which I have only praise. I especially appreciate the author's objectivity and willingness to entertain alternative explanations of his findings. For instance, in discussing possible proximate mechanisms responsible for his results, he is receptive to genetic, cultural, and structural-social explanations. I wish that all of my colleagues in sociology were as open as he to the major competing explanations for male-female differences.

Although all social and behavioral scientists should use the full range of genetic, cultural, and social explanations for the phenomena they study, my proper role as a sociologist commenting upon this study is to point out that some of the data are consistent with a structural-social explanation of the observed male-female differences. This evidence lies in an aspect of the findings that Buss does not fully exploit, namely, the variation in the measures among the societies represented by his 37 samples. Comparative family sociologists have often concluded that cross-societal variation in family forms (including marriage and mate selection) is related to level of economic development. Some aspects of family structure seem to relate to development in a curvilinear fashion, with preliterate and modern societies being similar and contrasting with agrarian ones (Blumberg \& 
Table 1 (Glenn). Relationship of indicators of development to Buss's measures

\begin{tabular}{|c|c|c|c|c|}
\hline \multirow[b]{2}{*}{$\mathbf{Y}$} & \multicolumn{4}{|c|}{ Product moment correlations } \\
\hline & 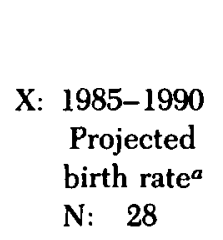 & $\begin{array}{c}\text { Log of per } \\
\text { capita gross } \\
\text { national } \\
\text { product, } \\
1983^{b} \\
22\end{array}$ & $\begin{array}{c}\text { Life } \\
\text { expectancy } \\
\text { at birth } \\
19\end{array}$ & $\begin{array}{l}\text { Less vs. } \\
\text { more } \\
\text { developed } \\
\text { countries }^{d} \\
28\end{array}$ \\
\hline $\begin{array}{l}\text { Importance of } \\
\text { financial } \\
\text { prospects of } \\
\text { spouse }\end{array}$ & & & $-.64^{* *}$ & \\
\hline Males & $.58^{* *}$ & $-.58 * *$ & & $-.67^{* *}$ \\
\hline $\begin{array}{l}\text { Females } \\
\text { Male-female }\end{array}$ & .70 & $-.43^{*}$ & $\begin{array}{l}-.63^{* *} \\
-.23\end{array}$ & $-.65^{* *}$ \\
\hline difference & .41 & -.06 & & -.23 \\
\hline $\begin{array}{l}\text { Importance of } \\
\text { industriousness } \\
\text { and ambition of } \\
\text { spouse }\end{array}$ & & & $-.59 * *$ & \\
\hline Males & $.63^{* *}$ & $-.47^{*}$ & & $-.68^{* *}$ \\
\hline $\begin{array}{l}\text { Females } \\
\text { Male-female }\end{array}$ & $.57^{* *}$ & $-.56^{* *}$ & $\begin{array}{l}-.59^{* *} \\
-.06\end{array}$ & $-.64^{* *}$ \\
\hline difference & -.14 & .15 & & .13 \\
\hline $\begin{array}{l}\text { Preferred age } \\
\text { difference } \\
\text { between self and } \\
\text { spouse }\end{array}$ & & & $-.81^{* *}$ & \\
\hline Males & $.83^{* *}$ & $-.46^{*}$ & & $-.62 * *$ \\
\hline $\begin{array}{l}\text { Females } \\
\text { Male-female }\end{array}$ & $.64^{* *}$ & $-.48^{*}$ & $\begin{array}{l}-.74 * * \\
-83 * *\end{array}$ & $-.63^{* *}$ \\
\hline mean & $.83^{* *}$ & $-.51 * *$ & & $-.67^{* *}$ \\
\hline $\begin{array}{l}\text { Importance of } \\
\text { good looks of } \\
\text { spouse }\end{array}$ & & & -.26 & \\
\hline Males & .25 & -.17 & & -.10 \\
\hline Females & .26 & -.30 & $-.40^{*}$ & -.23 \\
\hline $\begin{array}{c}\text { Male-female } \\
\text { difference }\end{array}$ & -.11 & .30 & .34 & .26 \\
\hline $\begin{array}{l}\text { Importance of } \\
\text { chastity of } \\
\text { spouse }\end{array}$ & & & $-.59^{* *}$ & \\
\hline Males & $.61^{* *}$ & $-.76^{* *}$ & & $-.73^{* *}$ \\
\hline Females & $.39^{*}$ & $-.69 * *$ & $-.44^{*}$ & $-.57^{* *}$ \\
\hline Male-female & & & -.37 & \\
\hline difference & $.62 * *$ & -.20 & & $-.47^{* *}$ \\
\hline
\end{tabular}

${ }^{*} p<.05 ;{ }^{* *} p<.01$.

a U.S. Department of Commerce (1987, pp. 818-19).

${ }^{b}$ Computed from data in U.S. Department of Commerce (1987, p. 824).

cU.S. Department of Commerce (1987, p. 820). Data are for various recent dates.

${ }^{d}$ U.S. Department of Commerce (1987, pp. 816-17). Variable is dichotomous.

Winch 1972; Lee 1982). Because the societies studied by Buss vary from agrarian to modern, the expectation is that among them measures of mate preferences will vary in an approximately linear fashion with development. I have accordingly correlated four indicators of development with each of Buss's measures for each sex and with the male-female difference or mean (in the case of preferred age difference for a spouse). The projected birth rate is a negative indicator of development, and the other three indicators are positive. For two indicators, I was able to use 28 of Buss's 33 societies, but for the other two, I could find appropriate data for only 22 and 19 of the societies, respectively. The results are shown in Table 1 . 
All of the indicators of development vary inversely with three of the five male-female differences. There is only one significant coefficient in the case of the importance of financial prospects, but there are two for the importance of chastity, and four for the preferred age difference between self and spouse. Except for the importance of good looks, all four indicators of development are inversely and significantly related to Buss's measures for each sex. According to the data, in the more developed societies as compared with the less developed ones, both males and females prefer smaller age differences between self and spouse, and both sexes place less importance on financial prospects, industriousness and ambition, and chastity of spouse. Even though only one of the six coefficients is significant, both sexes in the more developed societies may also place less importance on good looks.

These findings should be viewed with caution because of the small size and unrepresentative nature of some of the samples. However, random error should reduce the correlations, as should the kind of systematic error involved in sampling from the more modern portions of the less developed societies.

The correlations prove nothing and are subject to different interpretations. However, in the same way that Buss's analyses yield results that lend some support to evolutionary explanations of male-female differences in mate preferences (and perhaps especially to genetic explanations, although Buss does not say so), the results shown in Table 1 lend some support to structural-social explanations. They suggest (but do not prove) that development tends to diminish some of the male-female differences predicted by evolutionary theories. For instance, development may largely account for the lack of universality of the predicted male-female difference in the importance of chastity of spouse. And although all of Buss's 37 samples show the predicted male-female difference in the preferred age difference between self and spouse, the smaller mean size of the difference in the samples from more developed societies suggests that it may eventually disappear in some of them due to continued development.

The findings reported here in no sense discredit the evolutionary theories. However, if other studies should corroborate these findings, the theories may need to be modified to take into account their stronger ability to predict male-female differences in mate preferences in less developed societies than in more developed ones.

\section{Too many P's in the pod}

\section{John Hartung}

\section{Department of Anesthesiology, State University of New York Health}

\section{Sciences Center, Brooklyn, NY 11203-2098}

At 7:00 A.M. you can shoot the red light between Broadway and Driggs with only a 0.05 chance of getting caught. Unfortunately, if I took that chance every day for 37 days, my probability of running out of luck would be $1-(1-0.05)^{37}$, or $p>0.85$. Accordingly, if one wants a $<0.05$ probability of deriving false positives on 37 independent statistical tests of the same hypothesis ( $t$ tests or otherwise), the highest acceptable $p$ value per test is $1-(1-x)^{37}$ $=0.05$ (solve for $x$ ), or 0.00138 .

Also, if one adds a group of numbers and divides their sum by the number of numbers and then one subtracts that number from each individual number, sums the squares of those numbers, and divides that sum by the number of numbers and takes the square root of that, one gets two potentially meaningful numbers - the mean and the standard deviation. Like $6 \pm 2$ inches or $8 \pm 3$ pounds. One can cogitate the physical meaning of such an average and make probability statements, based on the measure of variability, about how well it represents the population from which the sample was drawn. For example, based on a sample of 60 , one can be $95 \%$ sure that the true mean of the population giving a sample mean of 6 inches and a standard deviation of 2 inches is between 5.5 and 6.5 inches. Given a second mean and sample variability, one can see whether the two means lie outside of each other's confidence intervals - i.e., one can do a $t$ test. And what if one is not dealing with inches or pounds, but is instead contemplating a difference between "indispensable" and "irrelevant" on a 4-point scale? It is difficult enough to think about (though easy enough to calculate) a mean of 2.4. It is more difficult to conceptualize a standard deviation of 0.82 , because one wonders: " 0.82 whats?" It is for this reason that a plethora of nonparametric ordinal tests have been devised for testing ordinal data. Nevertheless, although statisticians agree that using $t$ tests to compare ordinal "means" is logically meaningless, they do agree that it can be fairly safe empirically - that is, doing the job improperly can give the same false positive rate as doing it properly. In addition, it is done all the time in social science.

But empirical right and wrong is not a democratic matter. It has been shown that the assumption of linearity required to make it "safe" to treat ordinal data as if they were interval data breaks down slowly as the size of the scale goes from 20 to 11 , breaks down somewhat faster from 11 to 7 , and goes down the drain from 7 to 2 (Nunnally 1978). Buss's 4-point scale is clearly in the danger zone - not so much because it violates the assumption of normality ( $t$ tests are very good about that), but because, given unequal sample sizes from groups that are notorious for having unequal variability (e.g., female humans are more homogeneous on most physical measures), it violates the critical requirement of homogeneity of variance (Glass \& Stanley 1970). More specifically, even if people can linearly quantitate their feelings about an idealized dream-come-true's "previous experience in sexual intercourse," the measurement of the variability of people's feelings in this regard is so truncated by a 4-point scale that checking the validity of observed differences by $t$ tests is pointless.

So I am intrigued by Buss's results on preferred age differences (interval data, properly analyzed). My sense of it is that Williams (1975) was right in speculating that men will generally favor reproductive value over extant fertility in long-term relationships, and that Buss's results to the contrary are an artifact of the age of his respondents.

However, I cannot comment on Buss's other results. When 25 out of 37 inappropriately applied $t$ tests are not significant at the corrected alpha level, with 5 of those in the wrong direction (against the "chastity" hypothesis), I am left wondering what to think about. For "ambition and industriousness," 21 are not significant, and 3 are in the wrong direction. "Good looks" doesn't look much better.

Buss is probably right about each of his hypotheses. So what am I on about? Probably the vast majority of passersby, if stopped and asked the appropriate questions (e.g., "When looking for a mate, are looks more important to men, and money more important to women?"), would have come up with the same answers. (Social scientists are the only people who are confused about these issues.) Nevertheless, it is an important and worthy job to go out and empirically verify what might be only common (in both senses of the word) knowledge. This is an endeavor that is as inherently formal as it is formidable. In addition to methodological rigor (on which Buss cannot be faulted), it requires robust analysis. The whole job should be done right, or not at all. 


\section{Mating preferences surveys: Ethnographic follow-up would be a good next step}

\section{William Irons}

\section{Department of Anthropology, Northwestern University, Evanston, IL 60201}

David Buss is to be congratulated on an excellent piece of work. He has demonstrated the existence of some interesting and important sex differences not previously reported in the literature. This research demonstrates nicely the value of an evolutionary approach to the study of human behavior. Maccoby and Jacklin's (1974) comprehensive review of the literature on sex differences discovered none of the differences found here. Presumably, without evolutionary theory as a guide, it had not occurred to researchers to investigate these aspects of mate preference. Thus, among other things, evolutionary theory can be credited with drawing the attention of researchers to new variables and concepts. Not only are new ideas suggested, but often, as here, support is found for them.

One good way to extend this research would be to do ethnographic studies in the various societies included in Buss's survey. These ethnographic studies could attempt to identify the extent to which variation in social and cultural context can explain some of the observed variation in mate preference criteria. Could it be, for example, that the reversal of the usual difference in the male and female emphasis on ambition among the Zulu of South Africa reflects strong social constraints on male economic opportunities? Such studies might also reveal whether there is something unusual about the Spanish mating system which can explain the lack of the usual male-female difference in emphasis on earning potential. Are inherited wealth and status more important than "earning potential" in Spain? Or do Buss's survey data reflect unusual sampling error?

Perhaps the most interesting variable to look at ethnographically would be the one that showed the greatest crosscultural variability: the preference for chastity. A number of ideas relevant to chastity and the related variable of paternity confidence have been suggested. Alexander $(1974 ; 1979)$ suggested that males may respond to lowered paternity confidence by shifting investment away from wives' children to sisters' children, and there is empirical support for this idea (Flinn 1981; Gaulin \& Schlegel 1980) in the HRAF data. Perhaps the ability of men to do this makes it unnecessary to worry about chastity in certain contexts, or at least less necessary. Irons (1983) has suggested variation in the value of male versus female aid to a mother and the incompatibility of social arrangements encouraging both as a reason why women may not be interested in offering paternity confidence, and hence chastity, to a potential mate. These are possible explanations of the observed variation which could be investigated in ethnographic follow-up studies. I suspect, however, that although these ideas can explain variation among societies of the sort found in the HRAF, they will not explain variation among the more modern and urban societies that predominate in Buss's sample. These theoretical explanations are likely to find empirical support in societies in which extended families of various sorts are heavily involved in daily child rearing, but not in urban settings where such daily involvement in alloparenting is uncommon. My guess would be that males in modern, urban settings are concerned about cuckoldry and paternity confidence but they do not take previous sexual experience as a primary criterion for judging a potential mate's future fidelity. For different reasons - possible diversion of resources - modern, urban women are probably also interested in faithful mates. I suspect that careful ethnographic research, or perhaps more narrowly focused survey research, will reveal that in many modern, urban settings people are tolerant of sexual exploration before becoming committed to a mate, but expect fidelity after a commitment is made to a more permanent relationship. Under these conditions, I suspect that people evaluate probable future fidelity but do not take absence of past sexual experience as the main criterion for judging future fidelity. Exactly what the situation is, of course, is a subject for future investigation.

I agree with Buss that a more complete explanation of the differences revealed by his surveys will require studies of proximate mechanisms. I also believe that results of the sort presented by Buss will be a valuable guide to such work when it is undertaken. Buss mentions as potential proximate mechanisms to investigate "socialization" and "structural effects at a societal level." These are variables that could well be investigated ethnographically. Other proximate mechanisms such as "sensory preferences" could be studied best by the research techniques of psychologists.

I disagree with Buss's statement that we lack knowledge of the prevalence of arranged marriage in the environment of evolutionary adaptation. Although we cannot observe such environments directly, we do have a sound basis for making some inferences from anthropological research. We have a large number of ethnographies of preliterate and prestate societies. Included among these are a good number of studies of foraging societies. We also know from archaeological research that these ethnographically described societies are broadly similar in ecology, technology, and population densities to the societies in which our ancestors evolved. Given what we know, the most probable situation is that arranged marriages were the rule in the environments of evolutionary adaptation. However, having said this, I agree with Buss both that close kin are likely to make marriage arrangement conducive to the fitness interests of the parties to the marriage, and further that the parties to the marriage are likely to influence, in various ways, the decisions of their elders. It might be added that ethnographies of preliterate societies indicate that, although arrangement by senior kin is predominant for first marriages, the parties themselves are more likely to play the major role in arranging later marriages. Later marriages are common in preliterate societies because of the incidence of high mortality and, high divorce rates in some cases.

Buss points out very explicitly that his data tend not to include the rural and less educated members of the societies studied. I see this as no real problem. Other things being equal, evolved behavioral strategies should emerge more clearly in societies more like those in which human beings evolved. They should emerge less clearly in the urban, technologically sophisticated, less kin-oriented societies from which Buss's samples are drawn. These are, after all, evolutionarily novel environments. The fact that these strategies do persistently manifest themselves in these novel environments indicates that the phenomena are very robust. I, for one, would be very surprised to discover that a comparable survey of preliterate societies did not reveal the same mate preferences.

Buss has confirmed some straightforward predictions from sexual selection theory in an impressive cross-cultural study. $\mathrm{He}$ has demonstrated the existence of some sex differences in mate preference not previously reported. He has also raised a number of interesting questions for future investigation. I predict that his work will be the starting point for a number of future studies.

\section{Time to integrate sociobiology and social psychology}

\section{Douglas T. Kenricka and Richard C. Keefeb \\ Department of Psychology, Arizona State University, Tempe, AZ 85287 and 'Department of Psychology, Scottsdale College, Scottsdale, AZ 85275}

Like human sociobiologists, social psychologists have devoted a great deal of attention to sexual attraction and mating behaviors. Given that the first textbook in the field took an un- 
abashed evolutionary viewpoint (McDougall 1908), it may be surprising to read through the modern social psychological literature on mate selection and find almost no mention of the possibility that evolutionary considerations might have the slightest relevance to human mating patterns. Whenever sex differences emerge in this literature, as they often do (Kenrick 1987), they are dismissed as a function of the particular, and arbitrary, normative constraints of our own oddly sex-typed society. With his impressive data set, though, Buss adds to the mounting evidence that social psychologists went wrong when they rejected William McDougall's (1908) evolutionary perspective in favor of an exclusive commitment to more proximate explanatory models.

The fact that social psychologists have largely overlooked Darwin, however, is not a good reason for Darwinians to overlook social psychology. Contrary to Buss's remarks about our "profound" lack of knowledge concerning the proximate psychological mechanisms involved in mate choice, there is a whole subfield in social psychology that is focally concerned with these issues (e.g., Berscheid 1985; Brehm 1985). Social psychological findings provide a treasure trove of proximate puzzle fragments waiting to be pieced together within the ultimate perspective of evolutionary theory. We have reviewed some of these findings elsewhere (Kenrick 1987; Kenrick \& Trost 1988).

That said, it is clear that we are in basic agreement with Buss's evolutionary interpretation of his data. We do, however, wish to offer two qualifications from our own closely related line of research. First, a focus on criteria for a marriage partner may be least likely to show up evolution-based gender differences. Second, by reporting only the average preferred age of a mate, Buss may miss a distinction important to the evolutionary perspective.

Among social psychologists who study relationships, it is a commonplace observation that different processes operate at different phases of relationship formation (Kenrick \& Trost 1988). By focusing only on the selection of marital partners. Buss

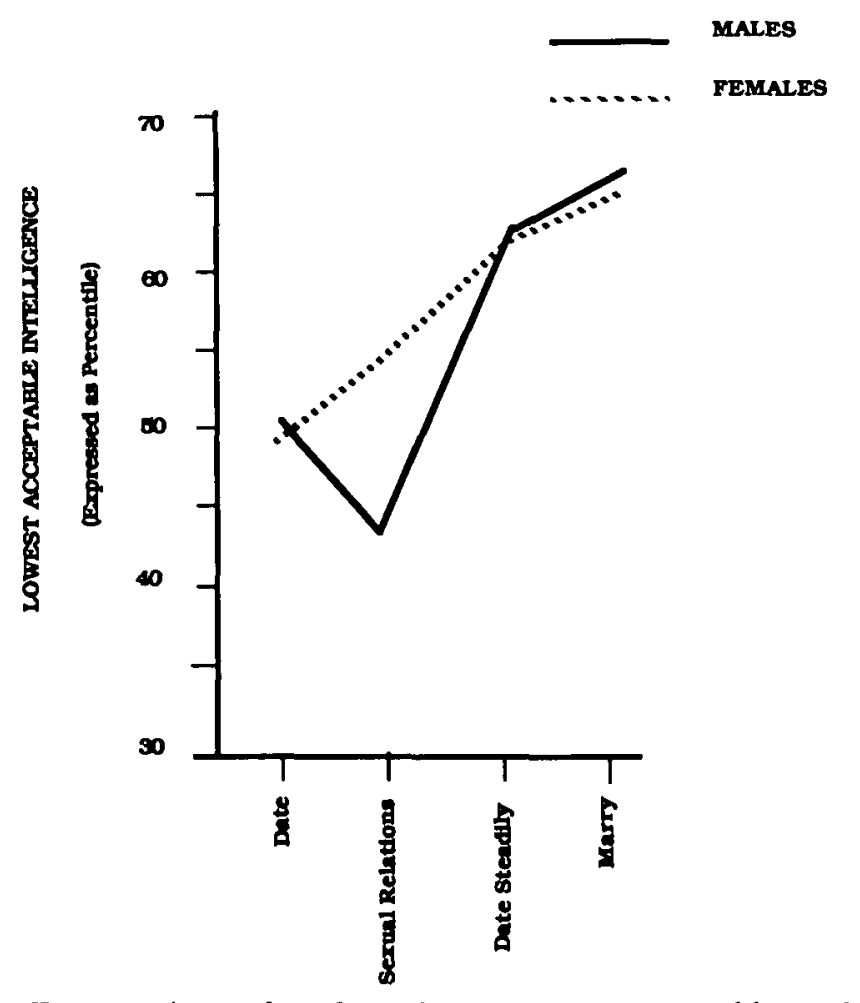

Figure 1 (Kenrick and Keefe). Minimum acceptable intelligence desired in a partner at four levels of involvement (from Kenrick et al., in press).

may have found fewer sex differences than if he had distinguished criteria for different levels of involvement. In fact, an evolutionary model leads to the expectation that gender differences should be least evident in choosing a marriage partner. The differential parental investment model (Trivers 1972) pre-

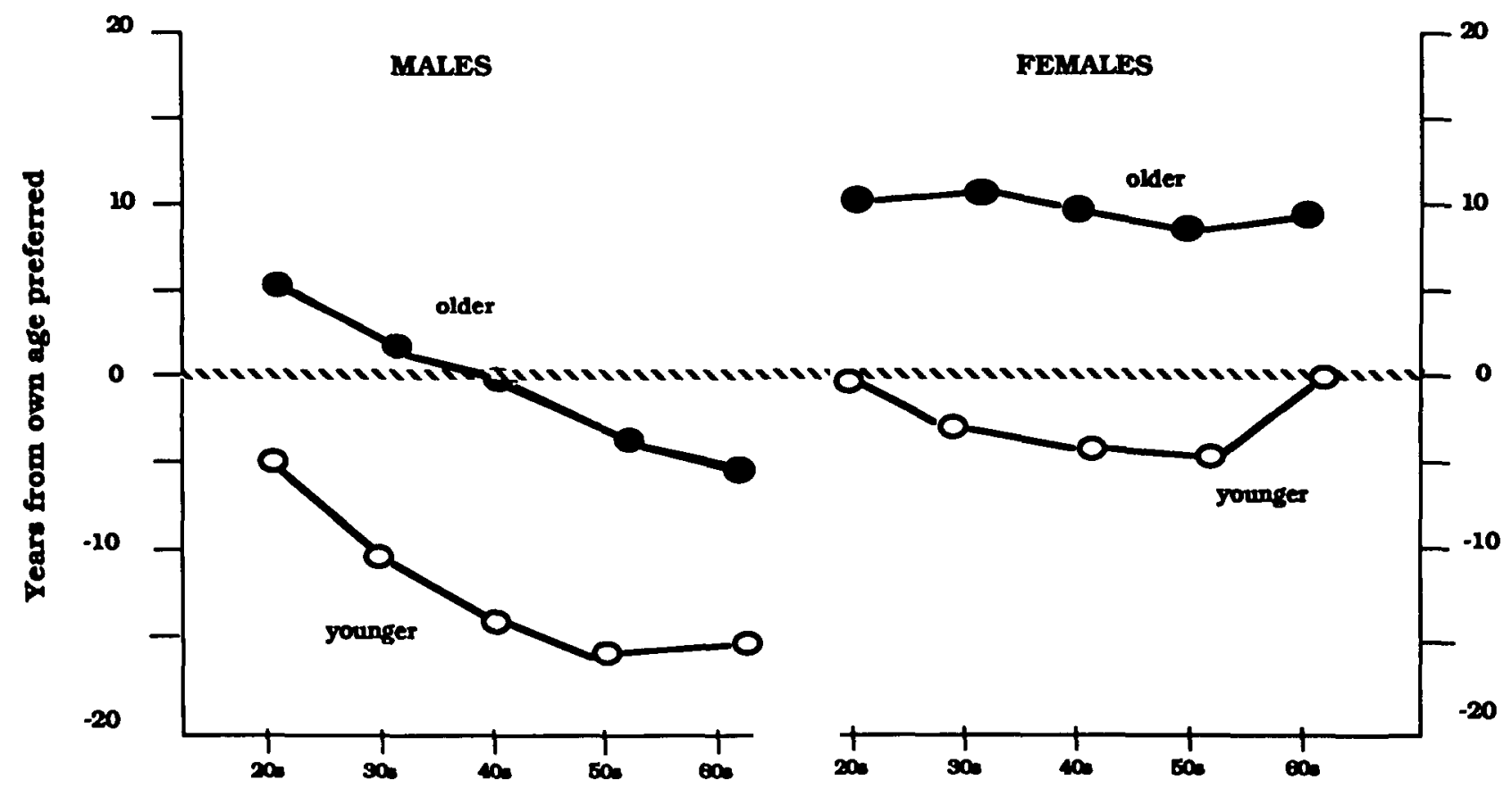

AGE OF ADVERTISER

Figure 2 (Kenrick and Keefe). Minimum and maximum ages specitied in dating advertisements placed by men and women, separated by advertiser's gender and decade of age. Dark circles mark the average upper limit of age preferred; open circles mark the lower limit. 
dicts that female mammals (who make a mandatory high investment in offspring) will generally be more selective than males (who may invest relatively little). Unlike males of many mammalian species, however, human males frequently invest heavily in their offspring. This should lead men to be highly selective about permanent mates. However, humans do engage in a number of illicit copulations (Daly \& Wilson 1983; Kinsey et al. 1948), during which males should be significantly less selective than females.

To test the evolutionary model in combination with the relationship-phase distinction often made by social psychologists, we asked American college students about their minimum criteria for a partner at four levels of involvement (Kenrick et al., in press). At the level of marriage, we found results generally in agreement with those of Buss. In particular, females demanded much higher standings on traits related to dominance and social status (like "earning potential"). The greatest sex differences, however, were found in criteria for a casual sexual partner. Figure 1 gives the results for the variable of intelligence. In general, our results suggest that males and females differ more in what they are willing to settle for in a casual sexual partner than in what they desire in an ideal mate.

On the second point, our results from another study (Kenrick \& Keefe, submitted) lead us to take issue with another of Buss's points - that regarding the desirability of a female marriage partner "just under 25 years" of age. Following a method used in several social psychological investigations, we conducted a study of the desired ages for a mate, as expressed in advertisements placed in a "lonely hearts" periodical (discussed at greater length in Kenrick \& Trost 1988). By arranging the data by the decade of age of the advertiser, we found a marked gender difference in the pattern of age preferences by decade. Females, regardless of their age, preferred a male who was, roughly speaking, between 2 years younger and 9 years older than they were. Males expressed very different preferences as they got older. Males in their twenties preferred a partner in the twenties. Older males preferred older partners, though increasingly younger than themselves. Thus, they seemed to prefer someone between their own age and the reproductive optimum (see Figure 2). These data are consistent with the evolutionary assumptions that Buss makes, but they show the problems in specifying an average age preference at 24.8 years. The age preferred seems to be confounded with the age of the sample under consideration. Men in their fifties, for example, commonly specified a partner in her thirties or forties.

In sum, we think that the theories, methods, and empirical findings of social psychology can be integrated with evolutionary models, to the mutual enrichment of both.

\section{Homo sapiens: A good fit to theory, but posing some enigmas}

\section{Janet L. Leonard \\ Department of Zoology, University of Oklahoma, Norman, OK 73019}

The results reported by Buss indicate that mate choice in Homo sapiens is about as would be predicted for a sexually dimorphic mammal in which the male provides some amount of resource to the female and/or his offspring. On the basis of cross-cultural surveys and comparison with other primates, particularly the apes, Smith (1984) concluded that humans have evolved in the context of a mating system where males are weakly polygynous that is, most males have only one mate, while some particularly high-ranking individuals have more than one - and females are monogamously pair-bonded to a male who provides parental investment, although facultatively polyandrous (i.e., extrapair copulations are not uncommon). In such species, females should favor males with attributes that are reliably associated with "good genes" and/or being "a good provider." Males should be somewhat "choosy" about the recipient(s) of this investment and will be expected to use criteria indicative of "good genes" and/or reproductive capacity. The data tend to be not inconsistent with this, although they are often difficult to interpret unambiguously.

Although Buss interprets the pervasive cross-cultural tendency for males to place greater value on physical attractiveness than do females (previously reported by numerous authors; e.g., Darwin 1877; Ford \& Beach 1951) as support for the prediction that males should choose a mate on the basis of reproductive capacity, it is not clear that the culturally variable qualities that constitute "good looks" (Darwin 1877; Ford \& Beach 1951) are reliably correlated with a woman's capacity for producing and rearing children. However, in a sampling of somewhat more diverse cultures, Ford and Beach (1951) found that there was some consistency across cultures in considering health and plumpness important components of physical attractiveness. Health would be important to a male looking for either "good genes" or high reproductive capacity, but traits attributable to substantial fat reserves (overall plumpness, large breasts, steatopygia, etc.) might offer particularly important clues to a female's current fertility and capacity to sustain a pregnancy and nurse a child. Furthermore, an interesting component of physical attractiveness in humans is the conspicuous role played by artificial traits (bound feet, make-up, jewelry, tattooing, etc.; see Darwin 1877; Low 1979) which would not be expected to be correlated with either "good genes" or high reproductive capacity. In summary, although Buss's results confirm those of previous studies, it is not clear that they support the hypothesis that males choose females on the basis of either "good genes" or high reproductive potential. The adaptive value of "beauty" in a mate remains unclear.

The data on preferences for age of mate are also difficult to interpret in a biological context because they are presented as a preference for a mate older or younger than the respondent. It is not clear that the relative age of the male and female is relevant from the standpoint of natural and/or sexual selection. A male choosing a mate on the basis of reproductive potential should prefer a female either of pubertal age (if reproductive value is most important) or in the early twenties (peak fertility), regardless of his own age. Buss considers the female preference for an older male to be unexpected, but I would argue that this is what would be predicted for a sexually dimorphic mammal. A female choosing a mate will want one that is reproductively mature, both physically and sociologically. In humans, as in many other sexually dimorphic mammals, males are slower than females in reaching both puberty and full adult growth (lagging by about 2 years; see Gardner et al. 1975; Katchadourian 1977). Therefore, if humans were to pair up monogamously as they reached reproductive maturity, one would expect the male tu be 2 years older than the female, on average. Furthermore, where males compete for mates, the more successful males will typically be higher-ranking. In complex societies, such as those of humans, there will often be a positive correlation between age and rank or resource-holding potential. Females, therefore, if they prefer "good providers," should show a preference for males who have achieved full maturity and high rank, which will typically mean older males, but perhaps males still young enough to retain effectiveness as providers until the females' last reproductive efforts have reached adulthood. If one assumes (conservatively) that a female marries at age 20 and can expect to bear her last child no later than age 40 , and that that child will require some degree of parental care until age 20 , then, if pairbonds are long-lasting, she should prefer a male who is currently a good provider and likely to remain so for $\mathbf{4 0}$ years. This would mean a male older than she is, but not much more than 10 years older. In this study, the preference shown by women in polygynous societies for husbands about 7 years older than themcelves may be explained that way. On the other hand, an older 
female choosing a mate, or a female choosing a male for extrapair copulation, might be predicted to show more latitude in relative age.

Buss predicted that males should value "chastity" in a potential mate because it would be a good predictor of certainty of paternity. It is not clear that this is a valid prediction. It is clear that a male should avoid marriage with a female who is pregnant with someone else's child; however, this could be done in ways other than by marriage with a virgin, because humans don't store sperm. A period of mate-guarding before marriage would fulfill this function, and there is no biological reason to consider that a female who is sexually experienced before forming a pairbond would be more likely to engage in extrapair copulations than a virgin would be. The importance attached, in many cultures, to female chastity cannot therefore be readily explained as having a biological basis as a confidence-of-paternity mechanism per se. (See Smith, 1984, for a discussion of the hypothesis that the hymen may have evolved in the context of sperm competition.) Male sexual jealousy and the double standard can, however, probably be adequately explained as adaptations for paternity assurance (again see Smith, 1984, for discussion).

Buss's study demonstrates, therefore, as others have done, that, to the extent that such sociological data can be reduced to biologically meaningful variables, humans fit the expectations of theory (Trivers 1972) fairly well. However, there are several features of human mating systems (and sexual biology) that require more detailed study before their biological significance can be understood.

\section{Another intriguing data bank for use in testing culture-related hypotheses}

\section{Walter J. Lonner}

Center for Cross-Cultural Research, Department of Psychology, Westem Washington University, Bellingham, WA 98225

The invitation to write a commentary on Buss's article came at an opportune time for me. Summer session at Western Washington University (WWU) had just started and, as usual, I was beginning to introduce a class of about 20 undergraduate students to the whys and wherefores of cross-cultural psychology. Topical coverage in this course concerns, among a host of topics, perception, cognition, abnormality and, conveniently, values and how they are related to cultural and ecological factors. So I welcomed the opportunity to use the Buss data in connection with other material I was planning to present, primarily as a teaching aid for the course and only secondarily as information, as Buss had intended, concerning a Darwinian explanation of mate preferences. In other words, I chose to focus on the large and interesting sample, on the mean scores on the different scales that were presented, and on how all of this somewhat peripheral information might be useful for a little impromptu data gathering and data-bank comparisons.
Digging out of the library the relevant articles mentioned by Buss, I duplicated the "campus values" questionnaire and administered it to 53 females (mean age of 23.9) and 26 males (mean age of 24.9) who were students in three different psychology classes. These are rather small samples compared with the ones used by Buss, but they are not out of line with typical sample sizes used in most research. About $95 \%$ of the students were single, and nearly all were white and life-long residents of the state of Washington. For all 18 items on the scale, the same values that Buss reported were used: $3=$ indispensable or extremely important; $2=$ very highly desired; $1=$ desired but not terribly important; and $0=$ irrelevant or not important.

Next, I calculated the mean scores for the same "target" items used by Buss: ambition and industriousness (item 9), chastity (item 10), good looks (item 14), and good financial prospect (item 17). Not bothering with statistical contrasts such as $t$-tests, I made some simple mean comparisons with the two U.S. samples reported by Buss (i.e., Mainland and Hawaii). The data are shown in Table 1.

The pattern of results among the mean scores reported by Buss is generally supported by a similar pattern among the WWU sample, but there is one big exception. Whereas males in the two larger U.S. samples were accorded slightly more "license" by their potential mates in the area of chastity (but insignificantly so) than by the potential mates of females, it was females at WWU who appear to want more "chaste" males. What does this mean? Does this sample of females fall outside a Darwinian framework? Is a social or cultural explanation more appropriate? Is it a statistical anomaly brought about by this sample? If so, how does one account for the absence of other departures from a fairly stable pattern amid the array of 24 means in the table? And the sample cannot legitimately be criticized, because Buss appears to be strongly in favor of diverse samples as a way to "increase the generality of consistent results that do emerge by minimizing the biasing effects of any particular sampling procedure."

Those considerations aside, I think that a major use of the data derived from this very large sample is to coordinate and/or compare them with other large, multicultural data banks that are now part of the cross-cultural archives. For instance, Hofstede (1980) has "values" data from about 115,000 midlevel managers from well over 40 countries employed by a large international company. He identified four factors: uncertainty avoidance (tolerance for ambiguity, the need for rules), power distance (essentially a "pecking order" factor focusing on superordination-subordination in the workplace), individualism (looking out for self and immediate family as opposed to the larger collective group), and masculinity (emphasis on assertiveness, activity, and things rather than humility, passivity, and people). Because many of the 33 countries in Buss's study are also in Hofstede's, considerable use could be made of the data banks for hypothesis testing, or at least for classroom purposes. For instance, shouldn't there be a strong, positive relationship between Hofstede's Masculinity Index and differences between the countries and sexes on the "chastity" item? (There seems to be.) Or between the Individualism Index and the desire for

Table 1 (Lonner). Mean scores on the four critical values items for males and females in three different samples

\begin{tabular}{|c|c|c|c|c|c|c|c|c|}
\hline \multirow[b]{2}{*}{ Sample } & \multicolumn{2}{|c|}{ Item 9} & \multicolumn{2}{|c|}{ Item 10} & \multicolumn{2}{|c|}{ Item 14} & \multicolumn{2}{|c|}{ Item 17} \\
\hline & $\mathbf{M}$ & $\mathbf{F}$ & $\mathbf{M}$ & $\mathrm{F}$ & $\mathbf{M}$ & $\mathbf{F}$ & $\mathbf{M}$ & $\mathbf{F}$ \\
\hline U.S. Mainland & 1.84 & 2.45 & .85 & .52 & 2.11 & 1.67 & 1.08 & 1.96 \\
\hline U.S. Hawaii & 1.95 & 2.24 & .91 & .58 & 2.06 & 1.49 & 1.50 & 2.10 \\
\hline $\begin{array}{l}\text { West. Wash. } \\
\text { U. }\end{array}$ & 1.73 & 2.18 & 1.11 & 1.74 & 1.54 & 1.09 & .81 & 1.20 \\
\hline
\end{tabular}


ambition and industriousness as a desirable characteristic in a prospective mate? (This also appears to be the case.)

An even more useful data set to use for contrasts, comparisons, and hypothesis testing is to be found in Williams and Best (1982). Their research concerns the measurement of sex stereotypes across cultures; the original (and currently expanding) sample included 2,800 university students from 28 countries. The main measuring device was the 300 -item Adjective Check List (ACL). Williams and Best determined, for example, all the items that were "stereotypically male" as well as "stereotypically female" across all cultures - quite a number of which fell in each category. Several of the adjectives are essentially the same as some of the phrasing used in the values scale used by Buss. On the ACL are the adjectives "ambitious," "industrious," and "good-looking" (which together cover two of the values items Buss used - namely "ambition and industriousness" and "good looks"), as well as "resourceful," which is fairly close in meaning to "good financial prospect." The Williams and Best data can probably be searched for many intriguing "evolutionary hypotheses," which apparently would be a new use for them. In addition, Williams and Best conducted ego-state analysis as well as psychological needs analysis based on Murray's need-press theory of personality. Their book is brimming with tables of means and other indices, by sex and by country, across the various psychological dimensions.

The three valuable sets of multicultural data should be explored in detail, for they are rare gold mines of potential insights. If something cannot be found in all these data to help Buss in his search for Darwinian linkages, then either he will not have looked hard enough or creatively enough or the two sets of data do not contain interesting parallel connections of the type I suspect are there for the taking. Buss, Hofstede, and Williams and Best need to get together to discuss the near certainty that their separate sets of data are complementary in many ways, and to outline testable hypotheses. This is one of the main values of Buss's data.

\section{The sociobiology of human mate preference: On testing evolutionary hypotheses}

\section{Nadav Nur}

Department of Biostatistics, University of Washington, Seattie, WA 98195

Ten years ago, in October 1978, a symposium was held at Ann Arbor, Michigan, on the topic of Natural selection and social behavior. The symposium included a handful of papers that applied the new and stimulating evolutionary theories of William Hamilton, Robert Trivers, and others to humans. The target article by Buss would have made an excellent contribution to that path-breaking symposium; instead it appears a full decade later. The study of the evolutionary biology of behavior has made many advances during the intervening years. Is this development reflected in the target article? I am afraid the answer is no. Buss purports to test evolutionary hypotheses derived from "recent theoretical work"; but the basis of the predictions essentially consists of papers from the early- to mid-1970s, the formative years of the new field of sociobiology. There is nothing deficient about these seminal papers, but to advance the field of sociobiology, we should strive to test strong rather than weak predictions and to interpret the results in a sophisticated fashion, relying on the progress made in evolutionary biology over the last decade.

Buss begins by contrasting male and female parental investment in humans. He characterizes parental investment primarily in terms of energy, resources, and so forth. Parental investment, however, was defined by Trivers in terms of the ability to invest in other offspring, a point made clear by subsequent theorists (e.g., Maynard Smith 1977; Partridge \& Halliday 1984). Thus, with true (complete) monogamy, the level of investment of the two sexes is equal, irrespective of energy investment, resource investment, and so on. Theory predicts, furthermore, that in a polyandrous mating system, females should be choosier! Whereas Buss attempts to derive a uniform prediction for all cultures, specifically that "females, more than males, should value attributes in potential mates such as ambition, industriousness, and earning capacity" a sophisticated application of evolutionary theory tests the prediction that variation in choosiness should be related to variation in factors such as the mating system. For example, we might predict that the greater the degree of polygyny, the greater the difference in the sexes with respect to choosiness. Buss in fact mentions that "Triver's prediction should apply only in contexts where resources can be accrued, monopolized, and defended, where males tend to control such resources, and where male variance in resource acquisition is sufficiently high," but Buss does not make use of this qualification to derive testable predictions.

Evolution-minded behaviorists of the presociobiology era looked for generalizations about humans that held across all cultures (e.g., Eibl Eibesfeldt 1970); modern evolutionary biologists incorporate variation into their theoretical frameworks: not only variation in causal factors but also variation in behavior, across cultures and within populations. The theory of evolutionarily stable strategies, pioneered by Hamilton (1971) and Maynard Smith (Maynard Smith \& Price 1973; review in Maynard Smith 1982), provides a good theoretical framework for understanding such variation (see, e.g., Dunbar 1982). The approach I advocate yields powerful predictions and thus sharpens our understanding of behavioral determinants. For example, consider predictions regarding reproductive value: The change in reproductive value with age varies among cultures; therefore, a strong prediction of evolutionary theory is that differences in preferred age of mate should correlate with differences in age of peak reproductive value or peak fertility (depending on the hypothesis being tested).

The relationship between culture and evolution is treated in a naïve fashion by Buss. For example, he finds that in some cultures females value ambition and industriousness more than males do; in some there is no significant sex difference; and among the Zulu, males value ambition and industriousness significantly more than females do. Buss "explains" the Zulu finding in terms of Zulu culture, but does not consider that the findings in other cultures (including those in which females value ambition and industriousness more than males do) might likewise be explained by culture. Thus these data do not provide strong support for Buss's evolutionary hypothesis; they do tell us that Buss's evolutionary theory alone cannot account for observed mate preferences with respect to ambition and industriousness. The hypothesis that culture alone determines mate preference does not predict a priori whether males or females will value industriousness more; in contrast, Buss's evolutionary hypothesis does. Thus, overall, the cross-cultural findings are consistent with the culture-alone hypothesis but inconsistent with Buss's hypothesis. A more sophisticated evolutionary hypothesis is that the value placed on ambition/industriousness depends on a number of social factors, for example, the degree to which women are dependent on their husbands for financial support (rather than, say, their families or their husbands' families). Such an hypothesis might be able to account for the degree to which either sex (in a given culture) values ambition or industriousness.

The roles of evolution and culture are again muddied with regard to the analysis of chastity preferences. About half the samples show no significant sex difference with regard to chastity preference; Buss concludes that these results provide "powerful evidence of proximate cultural influences" on chastity preference but "only moderate support" for the evolutionary hypothesis. The inference is that universality among cultures is associated with evolutionary determinants; differences among societies is evidence for cultural influences. I strongly resist such a dichotomy of evolution versus culture, 
which is as sterile as the nature/nurture dichotomy of years back. An evolutionary framework is as important for explaining differences among cultures as it is for explaining commonalities. For Buss, "the wide variation in preference for chastity suggests that cultural differences, ecological differences, or mating system differences exert powerful effects on the value attached to chastity." For me, a modern evolutionary hypothesis incorporates differences in culture, ecology, and mating system. In contrast, the search for species-typical behavior is anachronistic.

A modern evolutionary theory of human mate preference is waiting to emerge, it seems. (Recent work on the evolution of mate choice is summarized in Bradbury \& Anderson 1987; see also Pomiankowski 1987; Rice 1988; Tomlinson 1988; and references therein.)

\section{Mating behavior: Moves of mind or molecules?}

\section{Helmuth Nyborg and Charlotte Boeggild}

International Research Center for PsychoNeuroEndocrinology, Institute of Psychology, University of Aarhus, Risskov, Denmark

Buss deserves credit for quantifying cross-culturally an old saying that, in general, when it comes to mate preference, men value physical appearance more than women do, whereas women value resourcefulness more highly. We wonder, however, to what extent human mate preference is based on a rational choice; rather, mating behavior may have a molecular basis. Our point is that gonadal hormones, which exert quite specific and coordinated effects on the body and the brain and, accordingly, on behavioral traits, are the proximate causes of variations in mating behavior.

Buss is careful to admit that we know next to nothing about proximate mechanisms of mating behavior. His choice of terms indicates, nevertheless, that he embraces a traditional threelevel psychoevolutionary explanation. Females and males are said to "seek," "prefer," and "select," whereas parents may "choose" and "wish" on behalf of their children. Thus at the psychological level, Buss refers to mental qualities such as desires and values as immediate causes of mating behavior. At the level of interaction, Buss further suggests that genes and culture exert a combined effect on phenotypic mating behavior by assuming that (1) cultural differences may exert powerful effects on desires and values, (2) small or inconsistent sex differences suggest a cultural influence at the expense of genetic influences, and (3) large cross-culturally robust sex differences indicate a considerable genetic influence. Finally, with respect to evolution, Buss assumes that (4) mating behavior of the day reflects adaptations during primitive times to harsh but different reproductive constraints. In other words, evolutionary pressures were the remote causes.

For reasons given elsewhere, we agree with Buss that evolutionary pressures probably shaped our present mating behavior (Nyborg 1983; 1984), but we disagree with respect to the explanatory power of the first two levels of his three-level explanation. For example, how can natural or sexual selection possibly work on nonphysical mental categories such as desires and values? What are their mechanisms of action? Genes are pieces of DNA; how do molecules relate to mental categories such as desires and values? Where do genetic mechanisms converge with culture? To say that they coevolve is not to answer the question, but to raise even more complex questions. Perhaps genes and culture work through brain modifications! How, then, does the material brain relate to the nonphysical mind? And when in the evolutionary timetable of the brain did mind evolve? We fear that these unsolved (unsolvable?) bodymind problems easily drag our understanding of mating behavior into an explanatory cul-de-sac.
In order to circumvent the body-mind problems while retaining the explanatory power of Darwinian sexual selection, we have proposed the General Trait Covariance-Androgen/Estrogen (GTC-A/E) model (Nyborg 1979; 1983; 1984; 1986; in preparation). All sexually differentiated traits (bodily, brain, and behavioral - including mating behavior) are, according to the GTC-A/E model, manifestations of the proximate effects of preand/or postnatal variations in gonadal hormones on body and brain development, and remote consequences of evolutionary pressures. We further envision that the organization of the proximate mechanisms can be elucidated only by natural science methods. In addition, learning, evolutionary pressures, and other systematic environmental constraints are seen by the GTC-A/E model as nothing but changes in the physical environment, which must be coped with by adequate body and brain equipment in a strictly lawful physicochemical way in order to survive. The GTC-A/E model, therefore, incorporates neither desires and values nor nonphysical "social" and "cultural" factors in the attempt to explain mating behavior.

Such a materialistic idea is, most certainly, not new. The many predictions of the GTC-A/E model can now be tested with the help of new techniques of modern neuroendocrinology. It has been shown, for example, that microscopic changes in fetal and/or postnatal gonadal hormones may produce tremendous variations in the mating behavior of animals, with accompanying variations in their survival rate. Human mating behavior is obviously not a simple matter, and ethics prohibits experimental studies of humans to see how controlled variations in gonadal hormones affect mating behavior. However, the evidence from small clinical groups and from psuedoexperimental settings suggests, for example, that bisexual and homosexual behaviors contain hormonal components (Meyer-Bahlburg 1984; Rohde et al. 1978). Women with the adrenogenital syndrome or women who have been exposed prenatally to progestin are at a risk of developing atypical sexual behavior. Some men with an extra $\mathrm{Y}$ chromosome, and possibly with increased plasma testosterone (e.g., Schiavi ${ }^{+}$al. 1984), exhibit unconventional mating behavior coupled with lack of control (Theilgaard 1984). Career-oriented women seem to have more testosterone than have housewives (Baucom et al.1985; Purifoy \& Koopmans 1981), and they give birth to fewer children (Vining 1984). The GTC-A/E model predicts that testosterone switches women off their "safety-first" approach and onto a more masculine approach; it also predicts particular relations between gonadal hormones, frequency of intercourse, number of children, carrier prospects, and dominance-submissiveness. It has been observed that androgen increased libido in surgically menopausal (Sherwin et al. 1985; 1987) and normal (Bancroft et al. 1983) women. Moreover, men with karyotype $46, \mathrm{XY}$, but who are insensitive to their own androgens, develop in most respects like normal women, and unequivocally direct their sexual approaches toward men (Money \& Ehrhardt 1972). On the other hand, men who have been reared as girls because they were born with female genitalia and then develop male genitalia around puberty eventually direct their sexual behavior toward female partners despite their feminine rearing experience ( $\mathrm{Im}$ perato-McGinley et al. 1974). Finally, not only are men less "choosey" than women in matters of sexual behavior, but there also seem to be surprisingly large cross-cultural differences in mating behavior (Rushton \& Bogaert 1987) that, perhaps, can be explained by geographic variations in gonadal hormone secretion or sensitivity (Nyborg 1987; Soma et al. 1975). Taken together, these examples more than suggest that the proximate mechanisms behind Buss's interesting observations on mating behavior may be hormonal.

Researchers, realizing the complexity of studying the physicochemical basis of behavior, know that we are seeing only the tip of the iceberg. Yet this approach (which we call "physicology"; Nyborg, in preparation) seems more rewarding than the nonphysical, mentalistic approach, because mating behavior may be moves of molecules rather than of mind. The easily 
observable coordination by hormones of body, brain, and behavior (individual as well as interindividual) makes sense only within such a framework. Just consider that mating behavior appears only after hormones have primed the fetal brain and later matured the body. There would be no attraction and no one to be attracted to without the actions of gonadal hormones. Your loved one most probably has an estrogen-androgen balance opposite yours, but all sorts of intermediate solutions seem possible. The GTC-A/E model explains much of the intrasex variability in Buss's data as follows: Men and women with a relatively high androgen status will be attracted predominantly by physical appearance, whereas others with relatively low androgen status will perceive the resourcefulness of a potential mate as well. It seems that Buss's call for more research on the proximate mechanisms of mating behavior echoes in the corridors of already very busy neuroendocrinological labs.

\section{ACKNOWLEDGMENTS}

This work was supported by Grant 12-8300 from the Danish Medical Research Council and by Grant 15-6870 from the Danish Research Council for the Humanities to Helmuth Nyborg.

\section{Some psychoanalytic considerations}

\section{Daniel Rancour-Laferriere}

\section{Department of Russian, University of California, Davis, CA 95616}

Buss has made a significant contribution to the cross-cultural study of mate preference. He tests a set of hypotheses derived from an evolutionary or ultimate causal analysis of sex. He does not devote much attention to proximate mechanisms, however, particularly psychological mechanisms that must play a major role in mate choice. He even speaks of "our profound lack of knowledge about basic psychological mechanisms involved in human mating decisions." This statement, however, is debatable. Profound lack of interest in psychoanalysis might be part of the problem. [See multiple book review of Grunbaum's The foundations of psychoanalysis, BBS 9(2) 1986.]

Given the enormously expanded role of parental care in human (as opposed to other primate) ontogeny, it should in principle come as no surprise to an evolutionary biologist that the past experience of the parents has something to do with mate choice. In fact, however, the ontogenetic background of mate preferences has been neglected.

There is evidence, for example, that women use their ability or inability to have an orgasm with a man as a signal of whether or not that man will later provide resources to her and to her offspring (Rancour-Laferriere 1985, pp. 88-90). The most salient factor in the ontogenetic development of female orgasm, however, is past experience of the father (Fisher 1973; Uddenberg 1974). For purposes of a questionnaire, a woman may report that an ambitious/industrious man is what she wants, but for purposes of real interactions with men, she may in addition find out whether or not they are good prospects by discovering whether they produce the same good feelings in her as her father did. If her father was absent in her childhood, she does not have this mechanism at her disposal.

Male orgasm, too, is related to mate choice, and inevitably has an ontogenetic background. A man is more likely to ejaculate regularly with a woman whom he feels he controls than with a woman who is perceived as dominating him (as his mother used to in childhood). Indeed, he is likely to become impotent with an overly aggressive female, and a new mate choice has to be made if there are to be any offspring. What psychoanalysts term "castration anxiety" is perhaps one proximate mechanism in the service of a male's need to control the female(s) he is investing resources in - including the need to control female sexuality and assure certainty of paternity. Another function that castration anxiety may serve, however, is to enhance the reproductive value of the mate by making her younger than she would otherwise have been. Buss has certainly shown that males prefer younger mates. A younger female is less likely to be perceived as dominant than an older female, who may remind a man too much of the mother who used to boss him around.

From a psychoanalytic perspective, Buss's findings seem to indicate that the human female prefers a mate who is as much like her father as possible, that is, who invests in her, is older than she is (and whose looks are not too important), and so forth. The human male, on the other hand, seems to prefer a mate who is as unlike his mother as possible, that is, who does not invest in him, is younger than he is (and likely to be attractive), and so forth. In both cases, adult mate choice rests, in part, on past experience of the opposite-sex parent.

Some questions are raised by the fact, mentioned only in passing by Buss, that "both sexes ranked the characteristics 'kind-understanding' and 'intelligent' higher than earning power and attractiveness in all samples." What evolutionary hypothesis would have predicted such an important finding? What proximate mechanisms are involved? Is it implied that similarities between the sexes in mate preference are actually more important than differences? Here the psychoanalyst would observe that the elaborateness of mother-child interaction has greatly increased in the course of hominid evolution, and that adults of both sexes have learned to value kindness and intelligence specifically in the context of early interaction with the mother. Both sexes are looking for signals of future parental investment; what better mechanism for learning these signals than lessons learned from the mother - that is, the parent who invests the most?

There is much more that could be said from a psychoanalytic perspective, if only because so little has been said in the past (see also Badcock, forthcoming). Suffice it to say not only that psychoanalysis offers some potential proximate mechanisms for evolutionary biologists to consider, but also that the posited psychoanalytic mechanisms might themselves provoke new hypotheses concerning long-range evolutionary strategies.

\section{Preference for mates: Cultural choice or natural desire?}

\section{David C. Rowe}

School of Family and Consumer Resources, University of Arizona, Tucson. AZ 85721

The target article presents evidence that certain mate preferences are biologically biased. This evidence consists of the universality of cross-cultural sex differences in mate preference. The article, however, limits itself to emphasizing crosscultural evidence. Although the cross-cultural uniformity in the expression of a behavior is itself evidence for a biological basis to behavior - given the wide range of ecological conditions and social traditions among cultures - it is not wholly convincing. For example, the use of business suits is now nearly a crosscultural universal, at least in cities, yet one finds little evidence of a strong genetic compulsion to adopt this dress code.

Buss (1984) suggested several other criteria for evaluating biological dispositions: spontaneity, automaticity, intractability, and adaptation. In the case of physical attractiveness, the spontaneity/automaticity criterion applies: Infants apparently prefer the physically attractive, and this preference rules childhood play/groups, without any direct adult encouragement. Neither preference for age of mate nor preference for "earning potential" can, by definition, be easily detected in young children. Nevertheless, surrogate behaviors may be relevant - for example, physically coordinated children are more popular than others. In sum, because it follows just one line of argument, Buss's article omits other kinds of evidence in its favor. 
Even if one accepts Buss's general premise and regards mate preferences as partly an evolutionary heritage, several issues remain unresolved. One is clearly the nature of the proximate mechanism involved. Have brain areas evolved to promote a preference for the physically attractive? Such an idea is not so farfetched as it might appear. A brain area does exist that is specialized for facial recognition - and damage to it results in an inability to recognize faces. Moreover, Francis Galton's original suggestion that average facial features may be perceived as attractive - based on creating composite images of faces - could provide a basis for an attractiveness recognition mechanism (Plomin et al. 1980, p. 28). Similarly, although the trappings of wealth and power certainly vary among cultures, the idea of ranking is widespread in humans and animals; it is certainly possible for a mechanism to evolve that favors preference for high rank, where the system of ranking is learned. But these mechanisms are speculative. Unless evidence can be provided about the process linking DNA and behavior, Buss's thesis will be unconvincing to some scholars, and certainly incomplete.

The presence of individual differences remains a puzzle. Directional, evolutionary selection should eventually eliminate individual differences (e.g., humans, with rare exceptions, are two-legged). Yet, the target article notes that the "male and female preference distributions overlap considerably, in spite of mean differences." What is the origin of these individual differences, and why do they persist? Behavioral genetic studies of mate preference might be of interest here. Such studies could reveal no genetic influence on such preferences, indicating that the individual-level variation is merely the result of measurement error and idiosyncratic experiences. On the other hand, they might indicate that these preferences contain a component of additive genetic variation. Often, the latter type of variation suggests selective neutrality for a trait over evolutionary periods - genetically, it may not matter much whether one prefers a highly attractive or unattractive mate. However, under some circumstances - for instance, frequency-dependent selection strong selective pressures may exist and yet additive genetic variation will remain. Other complex selective schemes may also produce this result (Price et al. 1988). The presence of genetic dominance variation accords with the thesis of directional selection of mate preference. Finally, an absence of shared-environmental influences (by definition, environmental influences operating to make family members alike) supports the position that such preferences are not acquired from family environments or from other, local environmental influences.

Similarly, the genetic architecture of preference-determining traits is important. Little work exists on the heritability of physical attractiveness. Rowe et al.'s (1987) study of the facial attractiveness of monozygotic twins indicates a high broad-sense (i.e., all genetic components) heritability. However, the lack of other kinships (and a small sample size) prevents further partitioning of genetic variation in facial attractiveness. Traits related to "earning potential," such as I.Q. and a variety of personality traits, have strong, additive genetic components. If heredity plays a role in the determination of such traits, then the choice of mates possessing them should benefit one's offspring genetically - a mechanism that might promote the evolution of such mate preferences.

The target article also neglects the importance of rethinking mate preferences as biologically based dispositions. A large body of literature in social psychology and allied fields is devoted to the topic of choosing mates. In general, the theories describing the mate selection processes explicitly assume that mate preferences are consequences of other, social processes. For example, in the attribution literature, it is noted that many positive traits (e.g., friendliness) are attributed to the physically attractive. From this observation, it is argued that early socialization prepares people to make such trait attributions and then that such attributions determine mate preferences (e.g., for young and attractive mates). However, if these preferences were actually woven into the nervous system, and if they were emotions directly felt and determined by this biological substrate, then may not other, social correlates of mate preference be consequences rather than causes? Indeed, the entire attribution literature could be read as demonstrating the rationalizations people make for their inborn emotional responses. Thus, Buss's thesis has broad implications for many fields outside of evolutionary biology, including the psychology and sociology of mate selection.

\section{Epigenesis and social preference}

\section{J. Philippe Rushton}

Department of Psychology, University of Western Ontario, London, Ontario, Canada N6A 5C2

Evolution appears to have endowed humans with a discriminating desire for some as marriage partners rather than others. In addition, there seem to be mean differences between women and men in terms of which traits are considered attractive. Buss's data thus join those assembled in the domains of incest avoidance (see van den Berghe 1983) and sexual behavior (see Symons 1980) to support the view that human life histories are guided by epigenetic rules (see Lumsden \& Wilson 1982) acting in the service of fitness optimization. In this commentary, I extend this data base, suggesting that nature may have endowed individuals with a particularly fine-tuned set of social preferences.

Buss notes that other variables such as similarity might affect mate preference; elsewhere he has reviewed data showing that partners tend to resemble each other in such characteristics as age, ethnic background, socioeconomic status, physical attractiveness, religion, social attitudes, level of education, family size, intelligence, and personality (Buss 1985). Correlations tend to be higher for opinions, attitudes, and values $(0.40$ to $0.70)$, and lower for personality traits and personal habits $(0.02$ to 0.30 ). Less well known is the fact that partners also tend to resemble each other in terms of socially undesirable traits, including criminality, alcoholism, and psychiatric disorders. Alternative reasons can be proposed for this finding (Burley 1983), but it raises the possibility that the tendency to seek a similar partner may sometimes override considerations such as mate quality and individual fitness.

Genetic similarity theory (Rushton et al. 1984), an extension of the kin-selection theory of altruism, postulates that people detect genetic similarity in others (nonkin as well as kin) in order to proffer preferential treatment to those most similar. This suggests a new theory of attraction and friendship. Preliminary data make it likely that social assortment in humans is genetically mediated. It is known that the dimensions on which spouses and friends resemble each other are partly inherited (e.g., Tellegen et al. 1988); hence, unless one adopts the implausible idea that humans detecting similarity are responding purely to the environmentally influenced component of a trait, it follows that genetic similarity between partners must occur.

More direct evidence is also available. Using blood antigen analyses from nearly 1,000 cases of disputed paternity, Rushton (1988) found that degree of genetic similarity predicted (1) whether a pair was sexually interacting or randomly generated, and (2) whether a pair produced a child together or not. Seven polymorphic marker systems (ABO, Rhesus [Rh], P, MNSs, Duffy [Fy], Kidd [Jk], and HLA) at 10 loci across six chromosomes were examined. Sexually interacting couples were found to share about $50 \%$ of measured genetic markers, partway between mothers and their offspring, who shared $73 \%$, and randomly paired individuals from the same sample, who shared $43 \%$ (all comparisons significantly different, $p<.001$ ). In the cases of disputed paternity, genetic similarity predicted male 
inclusion: Males not excluded from paternity were $52 \%$ similar to their partners, whereas those excluded were only $44 \%$ similar $(p<.001)$.

Close friends are also found to be genetically similar using the same blood analyses. Rushton and Chan (in press) recruited 76 nonrelated close friends, ranging in age from 18 to 57 , from the general community. Subjects completed several life history, personality, and social attitude questionnaires, had numerous anthropometric measurements taken, and gave a blo d sample. The percentage similarity of the friendship pairs, as $u$ ell as that of an equal number of randomly paired individuals from the same sample, were calculated over the 10 loci. Whereas friends were found to be $54 \%$ similar on these genetic markers, the random pairs were found to be only $48 \%$ similar $(p<.05)$.

Although the blood antigens undoubtedly arise from assortment on related variables (there is no such thing as "genetic ESP"), the results are not due to obvious factors such as ethnicity and social stratification. Our samples were homogeneously North European in appearance (in the paternity study as judged from photographs), and with the friends, within-pair differences on variables such as education and occupation did not correlate with the blood tests. The critical point about these data is their demonstration that, in effect, social assortment often follows lines of genetic similarity.

Other data suggest that genetic influence on mate choice is particularly fine-tuned because within sets of homogeneous attributes, similarity between spouses is most pronounced on traits of high rather than low heritability. Several studies have found positive correlations between spouse similarity scores and estimates of genetic influence across a wide variety of anthropometric, cognitive, and personality characteristics (Rushton \& Nicholson 1988; Rushton \& Russell 1985; Russell et al. 1985). Rushton and Nicholson (1988) found these observations to be robust in that estimates of genetic influence calculated in one population (e.g., Japanese-Americans in Hawaii) predicted assortative mating coefficients in others (e.g., European-Americans living in California).

With friends, too, evidence exists that the tendency to choose similar others is itself genetically influenced. In Rushton and Chan's (in press) study, positive correlations were found between the differential heritability estimates and the degree of similarity between the friends (measured by correlation coefficients) on the attitudinal and personality assessments - that is, the friends' similarity was most marked on the more genetically influenced items, thus paralleling those from the studies of marriage partners. Other investigators independent of ourselves have also found evidence for genetic assortment. Rowe and Osgood (1984) used a behavior genetic design to examine delinquency in 530 teenaged twins and found that not only was antisocial behavior itself about $50 \%$ heritable, but that the correlation of 0.56 between the delinquency of self and the delinquency of friends was genetically mediated - that is, genetically disposed delinquent students were also genetically inclined to seek each other out.

Finally, parental preferences between full siblings have been examined. Because kin selection theory emphasizes relatives "identical by descent" where all siblings have a .5 coefficient of relationship, differences between full siblings has been overlooked. Because of assortative mating and the vagaries of meiosis, however, some children will be more similar to one parent than to the other. If a father provides a child with $50 \%$ of his genes, $10 \%$ of which overlap with the mother's contribution, and a mother provides the child with $50 \%$ of hers, $20 \%$ of which overlap with the father's, the child would be $60 \%$ similar to the mother and $70 \%$ similar to the father, and family members can be expected to favor those most similar. Support for this prediction was found in a study of bereavement following the death of a child: Both mothers and fathers, irrespective of the sex of the child, grieved most for children they perceived as resembling their side of the family (Littlefield \& Rushton 1986). Among siblings, perceived similarity is correlated with genetic similarity measured by blood tests (Scarr \& Grajeck 1982). [See also Plomin \& Daniels: "Why Are Children From the Same Family So Different?" BBS 10(2) 1987.]

It would appear that people do moderate their behavior in accordance with the genetic similarity of others. Thus human social preferences may be considerably more Darwinized than has been considered to date. As Buss notes at the conclusion of the target article, there is a need to understand the proximate mechanisms responsible for such effects. Like food preferences, sexual predilections can be highly individualized. Innate feature detectors, canalized learning, and idiosyncratic experiences may all have a role to play in ontogeny.

\section{Homo sociobiologicus not found}

\section{R. J. H. Russella and J. Bartrip}

apsychology Department, University of London, Goldsmiths' College London SE14 6NW, England and DMRC Cognitive Development Unit, 17/19 Gordon Street, London WC1E 7JG, England

There have been several theoretical approaches to the study of mate choice and marriage. Some are based on inadequate conjectures, some on the experience of clinicians, and some are derived from more broadly based theories of human functioning. The sociobiological approach is a relative newcomer, with several attributes that make it worthy of serious consideration. It draws its hypotheses about human beings from principles that have been found most useful in accounting for the behavior of animals engaged in a variety of reproductive strategies. It is unique in that the form of explanation is functional. Instead of searching for immediate causes, sociobiological theorists view behavior as playing a part in optimizing reproductive success. As the human marriage system is of such importance in human reproduction, this is an area to which sociobiological insights may be considered particularly applicable.

We accordingly welcome Buss's contribution. The derivation of hypotheses from basic theory is clear and convincing. The scale of the data gathering is impressive. By and large, the findings support the theory. However, the theory and the findings should both be taken with a pinch of salt. By its nature, the theory applies to an optimizing, ideal organism. It must be recognized that selection operates on a particular species with a particular set of behavioral possibilities. In contrast to Lumsden and Wilson (1981), who saw cognition as the link between natural selection and behavior, we contend that alterations in behavior are primarily selected through the proximal mechanism of emotional makeup. Sociobiological theorizing about human mate choice and bonding ignores the detailed study of the relevant emotions as its peril. Buss has paid little attention to the actual nature of the human in favor of purist, "species-free" theory. [See also multiple book review of Lumsden \& Wilson, BBS 5(1) 1982.]

Why should the findings be partly suspect? Because they rely on asking young people what they think they will want, rather than studying what people do. These reservations should not be taken to imply that we think Buss is wrong, but rather that what he describes is a facet of human mate choice. We feel that the term "mate choice," with respect to humans, is ambiguous. We assume mate choice to mean long-term mate choice, or marriage. With this in mind, we looked for corroborative evidence. We used some relevant questions from a comprehensive nationwide study of over 1,000 British married couples of all ages and socioeconomic levels. The data were gathered by Russell and Wells in 1986.

According to the target article, men tend to desire women who are young, chaste, and physically attractive, whereas women tend to want men who are wealthy and industrious. To obtain 
a measure of marital desirability, we summed two 5-point rating responses to the questions, "Do you think you were fortunate to marry your spouse?" and, "If you could choose, would you marry the same person again?" This sum formed the dependent variable. Six independent variables were calculated as follows: (1) Youth was determined simply from the person's age at marriage. (2) Premarital chastity was inferred from the response to the question, "How many people did you seriously go out with before you were married?" (3) Marital chastity was measured by the response to the question, "Do you find sexual fulfillment outside your marriage?" (4) Physical attractiveness was inferred from the sum of responses to "Do you think you are good looking?" and the spouse's reply to "Do you think your spouse is attractive to other people?" (5) Wealth was measured by the response to the question, "Were your parents well off?" (6) Industriousness was measured by the reply to "How hard do you usually work?" Although these measures were not designed for this purpose, we reasoned that, if Buss is right, youth, chastity, and attractiveness should be the main predictors of husbands' ratings of wives' desirability, and wealth and hard work should be the main predictors of wives' ratings of husbands' desirability.

Two multiple regressions were carried out, on husbands' and wives' desirability respectively. With a large sample and several predictors, a stringent significance level is appropriate. We chose the .001 level. In each analysis, the only significant predictors were attractiveness and marital chastity. Beta coefficients for the other variables did not even reach the first decimal place. The pattern of results admits of only one interpretation. Men and women both desire spouses who are physically attractive, and to the same degree (beta .248 for each sex). They dislike partners who are sexually unfaithful. Contrary to the hypothesis, women are more put off by unfaithful husbands (beta .217) than husbands are put off by unfaithful wives (beta .124). The results of these analyses provide no support for Buss's generalizations.

The data we used contain a number of other germane questions; for example, "Did you marry partly for financial reasons?" Here, Buss would be bound to predict that women would say yes significantly more often than men. They do not. Several other sex differences predicted by sociobiological theory likewise do not materialize; for example, "Are you jealous of your spouse's past relationships?" The dynamics of marriage seem to be driven by feelings of love, attraction, respect, and so forth, rather than by socioeconomic calculation - and in a way which is similar for both sexes.

Buss says that "little is known about precisely which characteristics in potential mates are valued by human males and females" (implying that his is the first major investigation of this type). This is not true. Substantial investigations (Richardson 1939; Spuhler 1968; Vandenberg 1972) going back to the last century have shown that what people want in a mate is someone like themselves (see Russell et al. 1985). This is the wellestablished fact that sociobiology, and indeed other approaches, must explain.

\section{Behavior depends on context}

\section{Robert W. Smuts \\ Evolution and Human Behavior Program, University of Michigan, Ann Arbor, MI 48109-1070}

The evolution of human mate preferences is more complicated than Buss's hypotheses and research design suggest. His first prediction is that women, more than men, should seek mates who control resources suitable for investment in children. As Buss points out, however, parental-investment theory starts with the fact that investment in offspring is more likely to come from mothers than from fathers. Among mammals, hard-working females usually support themselves and their young without male help. Whereas females invest additional resources in offspring, males tend to court additional mates; the more polygynous the mating system, the less males invest in offspring (Daly \& Wilson 1983; Trivers $1972 ; 1985$ ).

These generalizations are more true of humans than we often recognize. In spite of the spread of the monogamous ideal, most societies are more or less polygynous in the biological sense; that is, variance in reproductive success is greater for males than for females. Typically, among hunter-gatherers and in many traditional agricultural societies, women do most of the work and supply most of the economic resources they and their children (and their husbands) require. In the long view, the recent sharp increase in paid employment of wives and mothers throughout the developed world might well be regarded as a return to, rather than a departure from, the historical economic role of women. There are, in short, strong reasons to suspect that a preference for economically successful mates might often have a bigger genetic payoff for males than for females. When men fail to invest in children, their reproductive success requires mates who can and do.

The issue is further complicated by the fact that the ceiling on the ability to convert additional resources into additional offspring is vastly higher for males than for females. At least partly for this reason, variance in resource control is generally much greater among males. Males are likely to compete more vigorously and recklessly for resources because they compete for larger reproductive stakes (Hamilton 1966; Williams 1957). Consequently, the biggest winners and losers are more likely to be male. Whether male or female, rich people never suffer from a mate shortage, but when most of the rich are male, sexual selection for a rich-mate preference is strengthened in women and weakened in men, consistent with Buss's prediction.

One more set of complications is implicit in what has already been said. The sexual difference in the ability to convert resources into offspring creates a conflict of interest. Female reproductive success is best served by a reliably adequate flow of resources; male success depends more on maximizing the flow, even temporarily. Women might therefore be expected to prefer more industriousness and reliability and less ambition in their suitors. On the other hand, a woman may do a lot more for her long-run inclusive fitness (Hamilton 1964) than she can for her immediate reproductive success by marrying a man who dies rich and leaves his wealth to her sons. [See also Hartung: "Matrilineal Inheritance" BBS 8(4) 1985.]

Resource control is not, in short, a unitary concept, but may vary in several dimensions that are significant for the evolution of mate preferences. It can refer to reliability of resource flow, to quantity of resources, to the probability that resource expectations will be fulfilled, or to a term as short as the infancy of one child or as long as several generations. Buss's cross-cultural data do not lend themselves to analysis of cultural and sexual differences in mate preferences for different patterns of resource control. "Good financial prospect" and "good earning capacity" can apparently mean whatever the respondent thinks they mean, whereas attitudes toward "ambition" and "industriousness" cannot be distinguished, because the two qualities are linked as a single criterion.

The main point of these comments is that an evolutionary approach to behavioral science is not a search for behavior that is typical or universal for a species or a sex (Alexander 1979; Wrangham 1988). The basic message of evolution for behavioral studies is that behavior always depends on context; individuals tend to behave appropriately in the various and changing circumstances they encounter - with "appropriately" defined as whatever it takes to survive and reproduce (West-Eberhard 1987). All of this is recognized explicitly in Buss's discussion, but I suggest that recognition is not enough. The next step is to make 
this perspective the basis for hypothesis development and research design.

What evolutionists do best, by drawing on knowledge of other organisms and evolutionary principles, is developing testable hypotheses about the behavioral effects of contextual variables. I have suggested that human mate preferences are likely to be strongly influenced by local variance in the reproductive success of men and women, in resource control by men and by women, and in patterns of resource acquisition and retention. Buss acknowledges the importance of such variables, but neither his hypotheses nor his data are designed to sort them out. Space does not permit a consideration of Buss's other hypotheses, which are, however, equally broad and independent of contextual variation.

The cross-cultural consistency of Buss's results is interesting, impressive, and somewhat surprising. But it is hard to say how impressed and surprised we should be, because it is impossible to evaluate the diversity of his overall sample until the social and ecological correlates of mate preferences have been predicted and the local samples have been scored with respect to them. Because rural, uneducated, and poor people are underrepresented in his data, it may be that in spite of the worldwide disperson of his 37 samples, Buss really has only a few that differ in terms of the variables that matter most. His target article raises more questions than it answers, but that is perhaps the best thing an author can do when opening up a new, important, and challenging field of investigation.

\section{The psychology of human mate preferences}

\section{Donald Symons \\ Anthropology Department, University of California, Santa Barbara, CA 93106}

Buss's data are extraordinary: unique, hard-won, and valuable. I want to argue, however, that he underinterprets them. Although his data obviously cannot be used to identify and describe each of the myriad psychological mechanisms contributing to human mate preferences, neither is it true that "these results yield no information about the proximate . . . mechanisms directly responsible for their existence" (emphasis added). Were this statement accurate, Buss's selectionist arguments and laborious analyses of cross-cultural data would be pointless. Merely to ask whether data on some aspect of human affairs agree or disagree with an "evolutionary prediction" is, in itself, a scientifically empty endeavor: The answer will always be that they do or they don't, which seems to lead nowhere (Tooby \& Cosmides 1989). Such predictions are useful only insofar as the outcomes of tests of them augment our knowledge of "proximate mechanisms," that is, of phenotypic design (these points are elaborated in Cosmides \& Tooby 1987; Symons 1987b; 1989; and Tooby \& Cosmides 1989).

In predicting the existence of specific, universal criteria of mate preference, and sex differences therein, on the basis of selectionist reasoning, Buss does provide rough, preliminary hypotheses about "proximate mechanisms" (i.e., the psychology of human mate preferences), because the logic of his reasoning implies the following: (1) In ancestral human populations living in environments of evolutionary adaptedness (EEAs), heritable variation existed in some of the brain/mind mechanisms that underpinned mate preferences. (2) Individuals possessing certain variants and alleles out-reproduced, on the average, individuals possessing other variants and alleles specifically because the former tended to choose superior mates. (3) Some of the brain/mind mechanisms that underpinned mate preferences thus came to be shaped by selection specifically for that purpose (see Burian 1983; Williams 1966). (4) One can, hence, properly say that mechanisms specialized for mate preferences came to exist in human psyches and that genes for mate preferences came to exist in human gene pools. (5) Mate preferences, and sex differences therein, can be predicted - at least approximately - for modern human beings living in non EEAs, because modern human beings possess essentially the same mate-preference mechanisms and genes that their ancestors did (just as they possess essentially the same blood pressure regulating mechanisms and genes that their ancestors did).

The question of whether Buss's data and selectionist reasoning have implications about "proximate mechanisms" can be answered most easily by asking whether it is possible for his presentation to be incompatible with a nonevolutionary theory of mate preferences that ignores ultimate causation. In fact, his presentation is incompatible with virtually all such theories. Buss's data and selectionist reasoning imply that the assumptions about "proximate mechanisms" implicit in most socialscience theories of mate preference must be completely misguided. Social scientists typically attribute human mate preferences, and sex differences therein, to such things as "cultural conditioning," "socialization," "social learning," and "stereotyped sex roles" (see Symons 1979), all of which imply that these preferences are underpinned by some sort of generalized brain/mind mechanism (presumably of association or symbol manipulation); in other words, such theories imply that specialized mechanisms of mate preference do not exist (see Symons 1987b). (Buss's selectionist argument would be incompatible with traditional social-science notions even under a farfetched evolutionary scenario in which, for example, selection produced a sexually monomorphic brain/mind mechanism that specified the rule, "train your sons to be sexually attracted by unwrinkled female skin"; such a mechanism, however unlikely, would be no less specialized than a mechanism limited to male brain/minds that specified the rule, "be sexually attracted by unwrinkled female skin.") In sum, Buss's research is valuable precisely because it does have implications about "proximate mechanisms"; it would be even more valuable if he were to make these implications more explicit.

Let me illustrate these remarks with a concrete example. Because the average male in his sample prefers to marry a female just under 25 years of age, Buss concludes - albeit with a number of reservations - "that fertility has been a stronger ultimate cause of mate preferences than reproductive value," thus possibly falsifying a hypothesis of mine. But my actualexplicitly psychological - hypothesis was that human males have been designed by selection to be most strongly sexually attracted by physical characteristics (the precise details of which remain to be elucidated) that were reliably associated with maximum female reproductive value in EEAs (Symons 1979). ("Sexual attraction," being merely one component of mate preferences, must come closer to constituting a psychological mechanism/adaptation than "mate preferences" does.) By 25 years of age, most tribal women have had two or three children, and the evidence of childbearing, nursing, and rearing is clearly manifested in their bodies; women in industrialized societies where contraception is practiced can maintain a relatively youthful appearance far longer than is possible among women in tribal societies in which contraception is not practiced (Symons 1979 , p. 189). I strongly suspect that the men in Buss's sample who expressed a preference for a 25-year-old wife had in mind a nullipara (childless woman).

$I$ asked my colleague Napoleon Chagnon at what age females are most sexually attractive to males among the Yanomamo (an Amazonian tribal people whom Chagnon has studied for the past 25 years [see, e.g., Chagnon 1983]). He replied without hesitation, "Females who are moko dude." Chagnon explained that in the areas in which he worked, moko, when used with respect to fruit, means that the fruit is harvestable; when used with respect to a woman, it typically means that the woman is fertile. Moko $d u d e$, when used with respect to fruit means that the fruit is 
perfectly ripe; when used with respect to a woman, it means that the woman is postpubescent but has not yet borne her first child; she is probably 15 to 18 years of age. Nothing in my reading of the ethnographic literature on tribal peoples leads me to suspect that Yanomamo males are, in this respect, atypical (Symons 1979).

Despite Buss's findings, l'll hazard the following predictions: (1) Among tribal peoples who do not practice contraception, most men will be maximally sexually attracted by women who are moko dude. (2) Most men (including Western ethnographers) visiting a tribal people will be maximally sexually attracted to tribal women who are moko dude. (3) Among tribal peoples - but, for many reasons, not among industrialized peoples - men of all ages will consider women who are moko dude to be the most desirable wives. (4) Among industrialized peoples, most men will be maximally sexually attracted by women who are moko dude (although the effect of age on female sexual attractiveness will be far less marked than it is among tribal peoples, because women in industrialized societies can maintain a youthful appearance for so much longer). In the modern industrialized world, having sexual intercourse with women of this age is generally illegal and immoral, and even manifestations of such desires are stigmatized and penalized in subtle and unsubtle ways; hence, an imaginative research design would be required to test this hypothesis adequately and fairly. It is just this sort of research, however, that is most likely to zero in on the brain/mind mechanisms - the adapatations that collectively constitute the psychology of human mate preferences.

\section{ACKNOWLEDGMENTS}

Thanks to D. E. Brown and Napoleon Chagnon for their comments on this essay.

\section{Characteristics of female desirability: Facultative standards of beauty}

\section{Nancy Wilmsen Thornhill}

\section{Departments of Biology and Anthropology, University of New Mexico,} Albuquerque, NM 87131

David Buss has always impressed me with the ingenuity and thoroughness of his work. He continues to do so with this $B B S$ target article. The project described is fascinating and represents yet another example of the trustworthiness of intuition. (Surely no one has ever seriously doubted that men desire young, beautiful women and that women desire wealthy, highstatus men?) Nonetheless, empiricism is the necessary companion of theory. The empirical study at hand is impressive and would have proved quite daunting to many. Undoubtedly, that "problems were encountered, and data collection proved difficult and time consuming" can be classified as an understatement!

As full of admiration as I am for this work, I nevertheless have some comments, mostly to do with the author's interpretation of theory. First, I will take issue with the following statement:

Males should prefer attributes in potential mates associated with reproductive value or fertility, depending on whether males in human evolutionary history have tended to seek long-term or shortterm mating partners. . . . Specifically, if males in our evolutionary past have tended to seek short-term mating partners, selection should have favored male preferences for females in their early 20 s who show cues that are positively correlated with fertility. If males in our evolutionary past have tended to seek long-term mating partners, selection should have favored preferences for females in their midteens who show cues indicative of high reproductive value.

It seems that male standards of beauty have got to be more facultative than implied in the above-quoted passage. Throughout evolution human males must have chosen females for both long- and short-term mateships. Thus standards of beauty should have evolved to be context-specific, with the general rule of youth holding constant, rather than either reflecting reproductive value, or fertility concerns, or somewhere in between.

There is some empirical evidence (albeit somewhat indirect) that attests to a male psyche designed to evaluate beauty in the light of circumstances. Rape offenders appear to be somewhat more likely to choose as their victims women of high fertility as opposed to high reproductive value (Thornhill \& Thornhill 1983). Thus in the context of this fleeting mateship (from the offender's perspective), youth is evaluated, but even more telling, so is probability of current reproduction. Of course it is entirely unknown whether rape offenders desire women of high reproductive value for sexual relationships of a less temporary nature, but that is the prediction (Thornhill \& Thornhill 1983).

There are other data which indicate that men (and women too) are sensitive to perceived duration of relationship when evaluating desirable characteristics of potential mates (Kenrick \& Trost 1988). This study did not evaluate age preferences, but it did examine other mate qualities such as IQ, and found that men become less interested in the IQ of a mate (i.e., are happy to associate with women of relatively low IQ) as the duration of the mateship shortens (e.g., from dating to single sexual encounter). Again, this suggests that men use different standards of beauty (at least, desirability) depending on mateship duration.

In light of this consideration, some of the data that Buss views as anomalous might be reevaluated. Buss states that $38 \%$ of the 37 samples show no significant sex differences in valuation of chastity. These results provide only moderate support for the evolution-based paternity probability hypothesis." However, one can examine Table 1 , in which the mean ages of respondents are listed, and compare it to Table 6 , in which the mean scores for the chastity variable are listed. Taking male age 21 as an arbitrary cutoff point defining "young" men, we find nine samples in which mean male age was 21 or younger. Six of these nine represent samples in which no sex difference in value placed on chastity exists (South Africa [White], Great Britain, Greece, Ireland, Canada [English-speaking], and New Zealand). Furthermore, in one other sample of the nine where a significant sex difference does exist (Mainland U.S.A.), the mean value for chastity recorded by men is relatively low (.85).

Fourteen of the samples (38\%) show no sex difference in value placed on chastity. At least six (and possibly seven) of these reflect mean male ages of 21 or less. Perhaps when men are young (i.e., attempting to enter the breeding population and taking huge risks in the context of mating-effort expenditure), a woman's chastity is of little concern - in fact, is a hindrance. Young men may desire women who indicate their willingness to engage in easy, short-term, sexual relationships, rather than women who indicate a desire for long-term investment. As men age, and are perhaps more interested in mateships of longer duration, their criteria might change. These two situations would be expected to be reflected in male standards of female beauty (desirability).

In fact, some of Buss's other work gives support to this prediction. Buss (1988a, in another impressive study) found that, contrary to his prediction, behaving in a coy manner was not a character that college men found attractive in women, but behaving in a provocative, even promiscuous, manner was. Buss concludes that this finding indicates perhaps too much emphasis is placed on female coyness in evolutionary hypoth. eses about mate choice. I would argue that, in the male mind, female coyness is in fact a desirable characteristic under certain circumstances, to wit, when choosing a mate for long-term investment. I suggest that understanding male standards of beauty as facultative (condition-dependent) will be a far more predictive hypothesis than viewing the male mind as having evolved only a single standard.

Finally, I feel that to conclude "the male age preference for 
females [for marriage] of just under 25 years implies that fertility has been a stronger ultimate cause of mate preferences than reproductive value" is jumping the gun. Again, if male standards of beauty are facultatively expressed, prevailing conditions will determine preferred age of marriage partner (e.g., probability of divorce, tightness of monogamy, etc.). The conclusion would be warranted only if, when current conditions seem conducive to choosing women of high reproductive value (e.g., high paternity reliability, long-term mateships guaranteed, etc.), men still chose women of high fertility as mates. Finding this would surely imply a selective history involving only short-term (preference for high-fertility women) mateships. I don't know if the relevant ecological conditions can be discerned from Buss's data to evaluate the conclusion in this way.

Just as an aside, the age of peak fertility is somewhere between 20 and 25 for U.S. women (in 1972) but, even so, fertility drops almost imperceptibly between 25 and $30\left(F_{x}(20-\right.$ $25)=.13, \mathrm{~F}_{x}(25-30)=.12$ ) (Thornhill \& Thornhill 1983). So a preference for 25-year-old women still represents a preference for high fertility, not a preference for women "several years beyond peak fertility.

I began this commentary with praise for Buss's work, and I will end it on the same note. The work is careful and well thought out, and indicates a good understanding of evolutionary principles. More personality and social psychologists should follow Buss's lead.

\section{The innate versus the manifest: How universal does universal have to be?}

\author{
John Toobya and Leda Cosmides $b$ \\ aDepartment of Anthropology (Biological Wing), Harvard University, \\ Peabody Museum, Cambridge, MA 02138 and 'Department of \\ Psychology, Stanford University, Stanford, CA 94305*
}

With this target article and his other recent papers, Buss has provided a tour de force of empirical investigation into human mating preferences. As such, his work constitutes a major contribution to the effort to explore the evolutionary psychology of human mate selection and to the larger debate on the relevance of evolutionary biology to psychology. Claims that human behavior and human psychology have been shaped by our evolutionary history are claims about a universal human nature (with some rare exceptions; Tooby \& Cosmides, submitted). Consequently, the ideal approach is to test evolutionarily derived hypotheses cross-culturally, something that is rarely done, because of the logistical problems involved. Although no cross-cultural study ever approaches the ideal, Buss's work exhibits many virtues rare in cross-cultural research, including a good attempt to standardize data-gathering procedures. Crosscultural tests have become especially important because of the frequent accusation that the major evolutionary hypotheses (inclusive fitness theory, sexual selection theory, etc.) cannot withstand cross-cultural scrutiny, and are instead simply an expression of Western ethnocentrism projected onto the animal and non-Western world (Sahlins 1976).

But how universal does a universal need to be to establish something about human nature? This depends on whether one's hypothesis is about behavior or psychology. If the hypothesis is about universal human behavior, then devout Popperians will tell you that a single exception falsifies the universal claim. Anthropological traditionalists (although not Popperians in the sense of advancing falsifiable theories of their own) are, by and large, not much interested in the enterprise of searching for human universals. They are already familiar with the vast reservoir of variability within and especially between cultures. Even though evolutionarily oriented anthropologists return, again and again, with the finding that humans actually behave in a far more Darwinian fashion than a credulous acceptance of local ideology would lead the traditional ethnographer to believe, the skeptical anthropologist or critic can still point to enough variability to make the search for behavioral universals a lean and foolhardy pursuit. The standard Boasian rejoinder to those who make claims about a universal human nature is to point to cultures where things superficially appear otherwise (e.g., Mead's [1949] Male and Female). Within-culture variation also offers fertile ground for the skeptic: Suicides, homosexuals, celibates, the religiously inspired abandonment of worldly pursuits, and so on, offer apparent falsifications of claims about universal fitness striving. The result has been a standoff. Traditional anthropologists point to the complexly variable patterns of human behavior, while Darwinian anthropologists (in Symons's [1989] sense) argue for an evolutionary cynicism: that beneath the mask of bizarre cultural ideology lurk deceptive individuals manipulating their fellows to maximize their inclusive fitness.

If, on the other hand, one's hypothesis is about features of an innate, universal human psychology, then the claim of universality must be tested differently. Genetics had enormous difficulty making progress as a science until geneticists drew the distinction between genotype and phenotype: between the inherited basis of a trait and its observable expression. We believe that a similar distinction will be equally useful for an evolution-based psychology. We will refer to this as the distinction between an individual's innate psychology and an individual's manifest psychology and behavior. One observes variation between individuals and across cultures in manifest psychologies or behaviors; one views these as the product of a common, underlying evolved innate psychology, operating under different circumstances. The mapping between the innate and the manifest operates according to principles of expression that are specified in innate psychological mechanisms; these expressions can differ between individuals when different environmental inputs are operated on by the same procedures to produce different manifest outputs (Cosmides \& Tooby 1987).

This view is at least implicitly accepted by almost everyone involved in the debate on human nature. However, by making it explicit, one can clearly see that cross-cultural tests of evolutionary hypotheses depend directly on associated claims about the nature of innate psychological mechanisms, especially about the rules that govern their expression in various conditions. One useful distinction is Mayr's (1976) closed versus open behavior programs, referring to programs that are open to environmental inputs, and hence variable in expression, versus those that are closed to environmental input, and consequently uniform in expression. The search for universal human behaviors limits one to finding only closed behavior programs; however, the search for our evolved innate psychology allows and requires the mapping of all mechanisms, closed or open.

Yet, how can one distinguish between variable expression of an innate procedure and no mechanism at all? Fortunately, that is not the real choice. As Symons (1987b) has cogently argued, the real nature-nurture debate is between those who believe the human mind has many psychological mechanisms that are domain-specific and special-purpose (e.g., mate-choice mechanisms), and those who believe human behavior is the product of a few global, domain-general mechanisms (e.g., the culture theorists' hypotheses about culture-learning, norm imitation, etc.). Because all behavior is the result of some mechanism or set of mechanisms, the choice is not between a mechanism and no mechanism, but between one mechanism and another. In rejecting the hypothesis that domain-specific mechanisms govern mate choice, one is necessarily claiming that domaingeneral mechanisms account for the existing distribution of mate preferences - the implicit claim of culture theorists (Sperber 1984; Symons 1987b; Tooby \& Cosmides 1989).

In order to evaluate the claims of culture theorists empirically, we need to recognize that such claims entail theories about innate psychological mechanisms: in fact, equipotential mechanisms. If the mechanisms were not equipotential, they 
would operate differently on different domains, and would therefore not be domain-general. Is it reasonable to believe that our innate psychology is equipotential? What kind of predictions about cross-cultural distributions of mate preferences does such a family of theories entail?

Like the rain, the burden of falsifiable prediction about crosscultural variation (as well as specific claims about mechanisms) falls on the domain-specific and the domain-general alike. The evasive critical stance that only the evolutionarily oriented have to shoulder this burden is untenable. To date, culture theorists have confidently rested their case on what now appears to be uncertain ground: that manifest universality across cultures is the requirement that evolutionary hypotheses about human nature must meet, and that, on the other hand, any degree of cross-cultural variability establishes that the behavior in question is the product of "culture." The distinction between a universal innate psychology and individually variable manifest behavior renders this argument unfounded. Culture theorists are instead left with an uneasy relationship to the data on human cultural variation. Until they replace their present range of mechanism-agnostic theories with theories that specify how equipotential, domain-general mechanisms predict the statistical distribution of existing societies, culture theory as it stands predicts the null hypothesis: that differences between cultures are random with respect to evolutionary hypotheses and therefore that, for example, sex differences should occur as frequently in one direction as the other. The assertion that "culture" cxplains human variation will be taken seriously when there are reports of women war parties raiding villages to capture men as husbands, or of parents cloistering their sons but not their daughters to protect their sons" "virtue," or when cultural distributions for preferences concerning physical attractiveness, earning power, relative age, and so on, show as many cultures with bias in one direction as in the other.

Until then, Buss has provided a strong prima facie case that evolved, domain-specific psychological mechanisms regulating mate preference exist. His ranking of the relative uniformity of cross-cultural expression of such preferences provides an important clue that should help in mapping out the mechanisms' procedures: The more universal preferences are the product of more closed behavior programs, whereas the more variable are the expression of more open behavior programs. ${ }^{1}$

\section{NOTES}

1. Specifically, we would suggest that the high valuation of chastity, although by no means universal, recurs with enough regularity in independent societies to imply the involvement of a domain-specific mechanism, and therefore that reshaping the hypothesis is called for. We suggest that, other things being equal, men monitor and choose mates who are less sexually experienced or active than average. Where competition for husbands is intense, females and their kin will compete to signal such a relative lack of experience, driving the population average for female premarital sexual activity downward. Where women are economically more self-sufficient, women are less restrained by the need for male investment and can disregard such preferences on the part of men to a greater degree, driving the cultural average upward.

*Please address all correspondence to Leda Cosmides.

\section{Mate selection: Economics and affection}

\section{Kim Wallen}

Department of Psychology and Yerkes Regional Primate Research Center Emory University, Atlanta, GA 30322

Electronic mail: Kin @emoryul.arpa

Buss argues that differences between males and females in their ratings of the importance of several hypothetical mate characteristics support the evolutionary interpretation that the rating patterns result from different selection pressures operating on males and females. Although the gender differences in the data are generally in the direction predicted by Buss, and it is tempting to conclude that substantial support has been found for an evolutionary interpretation of factors influencing human mate selection, the case is far from clear

Before concluding that an evolutionary perspective has been supported by these data, four matters must be addressed. First, the fit between the evolutionary hypotheses under test and Buss's results must be assessed. Second, an evolutionary explanation must be shown to be at least as good as, if not better than, alternative explanations. Third, the relationship between a person's stated preference on the items used and the characteristics of the actual mate selected must be determined. If the preferences reported do not substantially match the mates selected, then these ratings are of little use in advancing evolutionary arguments. Fourth, the hypothetical mate characteristics used in the study must be shown to be related to posited reproductive characteristics. In other words, if "good looks" do not accurately predict female reproductive potential, then a male preference for "good-looking" females is interesting but irrelevant to an evolutionary argument. Buss addresses the first of these items, showing a reasonable fit between his data and his hypotheses. The other three are largely unaddressed, except that data are presented showing a correspondence between preferred age difference in mates and actual age differences. Alternative explanations for his results are not tested, and the relevance of the items used as predictors of reproductive characteristics is not addressed.

A primary alternative explanation for Buss's results is that they stem specifically from the culturally determined economic status of women. Thus they do not reflect the action of selection pressures, unless one is willing to argue that all human economic and political systems result from natural selection, a position that Buss is clearly not advocating. From a cultural perspective, male economic control makes male economic status critically important to females in selecting a mate, because it ensures her economic future and that of her offspring. In turn, females, lacking economic influence, may use their sexual attractiveness as an avenue to economic and social power. Similarly, males may use their higher economic status to gain sexual access to females. Thus males may value "good looks" morc highly than economic potential in mate selection because females in most present cultures offer more of the former and less of the latter. This relationship might be different in dowryoriented cultures, or where females have gained a substantial measure of economic parity. In this regard, it is interesting that the results from Iran and the Asian nations most strongly support Buss's hypotheses and that these are also countries where women have low economic status. Because both cultural and evolutionary perspectives predict that the same characteristics are important in mate selection, more extensive data and more comprehensive analyses than those presented by Buss are needed.

One approach is to analyze the relative contribution of culture and gender to the ratings by grouping countries in terms of common cultural traits instead of analyzing each country separately. To illustrate this suggested analytical approach, I compared Buss's mean male and female ratings for each country on each of the five reported attributes ${ }^{1}$ using an analysis of variance that had gender and geographical origin ${ }^{2}$ as factors. The analyses addressed two questions. First, to what extent are the gender differences obtained using $t$-tests confirmed when the variance between countries is included in the analyses? ${ }^{3}$ Second, which factor (gender or geographical origin) accounted for the largest amount of nonerror variance in the ratings? Both gender and geographical origin significantly $(p<.02)$ influenced all five items. However, the percentage of the total variance each factor accounted for varied across the items. For the measures of "financial prospects" and "good looks," gender accounted for $45.3 \%$ and $40.2 \%$ of the variance, respectively, whereas geographical origin accounted for $17.1 \%$ and $8.3 \%$. For the measures of "chastity," "ambition," and "preferred age difference," gender accounted for $4.7 \%, 16.2 \%$, and $10.6 \%$ of the variance, 


\section{Average Rating}

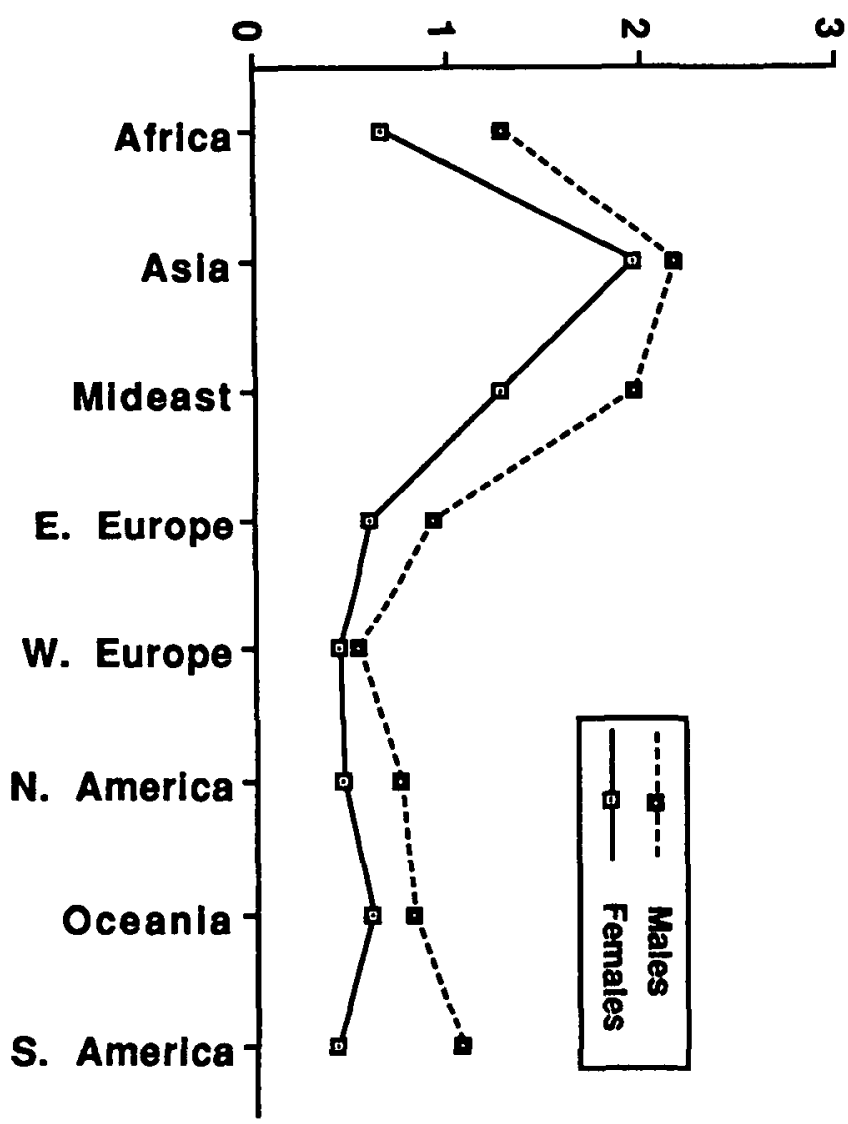

Figure 1 (Wallen). Average rating for the item "chastity" by males and females from various geographical regions.

whereas geographical origin accounted for $59.4 \%, 44.3 \%$, and $33.7 \%$ of the variance, respectively. Figure 1 illustrates the average ratings for the item "chastity" and shows the gender and geographical variation in this measure.

In sum, of the five measures reported, gender accounted for the largest part of the variance in two measures, and geographical origin in three. This suggests that a cultural perspective may be more powerful than an evolutionary perspective in interpreting these data. Analyses using all the data and cultural, instead of geographical, groups could readily provide the needed evidence.

To evaluate competing explanations, multiple aspects of the data should be compared. In this study, subjects ranked 18 items, including such items relevant to mate selection as "sociability" and "dependable character." The subjects' ratings of these variables are not reported, even though they could provide evidence of the value of psychological or emotional factors as determinants of human mate selection. It is surprising that in an article discussing the determinants of human mate selection, there is no mention of these items or others such as affection, nurturance, or dependability. Perhaps such characteristics do not readily lend themselves to sociobiological hypotheses. However, it would be hard to argue that they are irrelevant to human mate selection. Human mate choice may involve selection pressures acting on males and females, but it is most certainly influenced by proximal emotional processes.

\section{ACKNOWLEDGMENTS}

The critical discussion and comments of Dr. John L. Wallen are gratefully acknowledged.
NOTES

1. To make the item of preferred age difference similar to the other measures, the signs were removed. Subjects in all countries clearly preferred the male to be older. Removing the signs from the data allowed assessment of whether they differed in the magnitude of the age difference.

2. Countries were grouped according to Buss's categories, except that a Middle-Eastern grouping of Iran and Israel was created separately from the Asian grouping. Although not truly representing cultural regions, these geographical groupings reflect broad cultural differences.

3. Because the means for each country were used, subject variance was eliminated, making it more likely that significant effects would be found.

\section{Mate preference is not mate selection}

\section{Ada Zohar and Ruth Guttman}

Department of Psychology, Hebrew University, Jerusalem 91905, Israel

Buss's cross-cultural study, encompassing so many cultures and countries, is welcome and indeed necessary to substantiate any hypothesis about human evolution. Our main criteria is that evolution can only be affected by actual mate selection. Preferences are fascinating, both psychologically and sociologically. However, unless mates are actually chosen according to the stated preferences, these preferences are irrelevant to evolution. Buss attempts to bridge the gap between preferences and choice by a validity check on the preferred age difference between mates and mean spouse age difference in the population. In those populations where the data are available, there is no difference between the stated preference of the subjects and the average spouse difference of the population. The most likely explanation for this perfect fit is that when people are asked hypothetically about mate preferences, they reflect the norms of the culture. These norms are also expressed in the actual mean of the age difference in the population. This does not imply that individuals will, in fact, act according to these norms. Most likely, their choice will be affected by many variables, some of them personal, some situational (Buss \& Barnes 1986). Thus, the findings of the present study can be accepted as a reflection of the cultural norms of men and women regarding their mates in a variety of cultures.

Actual mate selection. To test sex differences in mate selection, it is important to ask people about the actual choice they made, as near as possible to the time of choice. To do this, one must turn to married people, so as to avoid the dropout of engaged couples who decide against marriage. One must reach the couples early in the marriage, before their life together has affected their view of their considerations for marriage.

In a sample of 51 couples in Jerusalem who had been married for less than a year, we asked the subjects to rate nine items on a scale of one to five for their importance in marriage considerations. The items were adapted from the classical study of Burgess and Wallin (1953) and were pretested on 450 couples. The newlyweds were contacted through university-owned "wedding halls," which are rented for special occasions to students, faculty, alumni, administrative and technical staff. The population sampled would typically have at least a high school education and would be rising socioeconomically. Thus, although our results cannot be generalized to the population as a whole, our sample - of a middle-class, upwardly mobile population - is similar to the samples used in most other mate-selection studies (Epstein \& Guttman 1984). Table 1 presents marriage considerations in order of importance to women. As can be seen, rank orders are nearly identical for males and females.

Women rank romantic love as the most important and setting up a home second; men, too, rank these items first and second, but in the reverse order. The only other difference in rank order is that women rate pleasing their parents higher than complying 
Table 1 (Zohar and Guttman). Reasons for getting married

\begin{tabular}{|c|c|c|c|c|c|c|}
\hline & \multicolumn{2}{|c|}{ Mean } & \multicolumn{2}{|c|}{ SD } & \multirow{2}{*}{$\begin{array}{l}\text { Discrimination } \\
\text { coefficient }\end{array}$} & \multirow{2}{*}{$\begin{array}{l}\mathrm{F} \\
\text { (variance } \\
\text { ratios) }\end{array}$} \\
\hline & Men & Women & Men & Women & & \\
\hline Romantic love & 2.02 & 1.82 & 1.2 & 1.2 & 0.17 & 0.69 \\
\hline Set up home & 1.98 & 2.35 & 1.0 & 1.4 & 0.28 & 2.20 \\
\hline Have children & 2.42 & 2.53 & 1.4 & 1.5 & 0.07 & 0.14 \\
\hline $\begin{array}{l}\text { Please your } \\
\text { parents }\end{array}$ & 4.16 & 3.92 & 1.0 & 1.4 & 0.19 & 0.98 \\
\hline Social mores & 3.90 & 4.18 & 1.4 & 1.0 & 0.22 & 1.30 \\
\hline Loneliness & 4.18 & 4.41 & 1.2 & 0.9 & 0.23 & 1.20 \\
\hline $\begin{array}{l}\text { Escape your } \\
\text { family }\end{array}$ & 4.48 & 4.78 & 1.0 & 0.6 & 0.47 & 3.19 \\
\hline Money & 4.60 & 4.77 & 0.95 & 0.55 & 0.30 & 1.12 \\
\hline Business & 4.64 & 4.78 & 0.90 & 0.64 & 0.28 & 0.85 \\
\hline
\end{tabular}

1-Very important

2-Important

3-Of certain importance

4-Quite important

5-Of no importance

with social norms, whereas, again, men reverse the order of importance. The mean standard deviations are very similar, and the sex differences are very small. Two measures of the sex differences are given in Table 1 . The values of the $F$ function are all very low - below 3.20. The other measure is the discrimination coefficient (DISCO for short), or $\mu 2$, which is a measure of discrimination between distributions; it ranges from zero, to total overlap between distribution, to one: no overlap or complete discrimination (Guttman 1981; 1988). As can be seen, the DISCO values are low, ranging from 0 to 0.40 . Thus, the striking finding is that in this sample men and women show very similar preferences in mate selection when asked about their actual mate choice.

There seems to be an inherent limitation in asking subjects directly about their preferences in mate choice, as social desirability may influence people's answers. In Buss's study, this may be a problem; it can be seen as a limitation of the Jerusalem sample as well. Note that in Table 1, the "positive" considerations are rated higher than the "negative" ones for both sexes, so that loneliness, escaping one's family, money, and business come after romantic love, setting up a home, having children, and so forth.

An alternate approach to sex differences in mate selection would be an indirect one, designed to overcome the bias of social desirability. One such indirect approach, which has not been tried and may prove fruitful, would be to look at the spouses of newlywed monozygotic (MZ) and dizygotic (DZ) twins. We would then have the advantage of testing the spouses rather than asking their mates about their salient characteristics. Because we know the genetic similarity of the MZs and DZs, we can then also assess genetic and environmental factors in mate selection. Sex differences can be approached in two ways: by comparing the choice of male MZs and DZs with those of female $M Z s$ and $D Z s$, and by comparing the choice of different-sex DZs. The genetic factors can be assessed by comparing the similarity of choice of $\mathrm{MZ}$ and $\mathrm{DZ}$ same-sex twins. [See also Plomin \& Daniels: "Why Are Children in the Same Family So Different" BBS 10(1) 1987.]

In summary, although we see limitations in Buss's approach and have offered two alternative research methodologies, we want to stress the great importance of a cross-cultural approach for understanding basic human processes such as mate selection.

\section{Author's Response}

\section{Toward an evolutionary psychology of human mating}

\section{David M. Buss}

Department of Psychology, University of Michigan, Ann Arbor, MI 48109-1346

Electronic mall: david buss@um.cc.umich.edu

It is a truism that there is a poor ratio of empirical data to theory in the emerging field of evolution and human behavior. The goal of my study of mate preferences was to improve this ratio. Because cross-cultural tests are often crucial for testing evolution-based hypotheses (Tooby \& Cosmides), I gathered data from many different countries around the world - indeed, from as many as I could over a 4-year period. The samples from 33 countries, with a total number of subjects of 10,047 , must be viewed as credible by any standard in the social and natural sciences. The empirical procedures were brief; the aims were limited; the probes were of necessity coarse-grained.

The study was designed to test several major hypotheses about sex differences in reproductive strategy that have been proposed in the evolutionary literature over the past 17 years (Daly \& Wilson 1983; Symons 1979; Trivers 1972; Williams 1975). Prior to this study, there were no extensive tests of these hypotheses on humans on an international scale. This study fills a gap. 
Before turning to the fascinating array of questions raised by the commentators, the following is a summary of what the study provides, whatever its flaws:

1. Powerful cross-cultural evidence of the prevalence of sex differences in mate preferences

2. Strong support for several specific evolutionary hypotheses that have been advanced over the past 17 years pertaining to male and female reproductive strategies

3. Support for the general hypothesis that males and females differ in reproductive strategy

4. Empirical data that must be accounted for by any theory of human mating behavior, whether evolutionary or nonevolutionary

These basic conclusions, with several important qualifications, are not disputed by the commentators of the target article. Prior to this study, it was simply not known whether human males and females, residing in countries throughout the world, differed consistently and predictably in their preferences for particular mates. Given the number of nations in which these sex differences were found, the burden of empirical proof now shifts to those who still maintain that human males and females are psychologically monomorphic with respect to mate preferences (Symons).

None of the commentators deny the fact of the crosssocietal sex differences documented, but this was not always the reaction prior to this study. Several years ago, after presentation of a series of studies on mate preferences within the United States (Buss 1985; Buss \& Barnes 1986), a frequent comment on my findings about sex differences was: "I can believe that these sex differences exist within the United States, or perhaps even within Western cultures, but these results are produced by our specific culture; surely they will not be found in other cultures." It was in part to respond to this kind of comment that $I$ initiated an investigation of 37 societies around the world to find out how pervasive these effects were.

Now that the results are in, I find it puzzling that several commentators insist that (1) these sex differences are still accounted for solely by "culture" in spite of their prevalence across cultures (Wallen, Zohar \& Guttman), and (2) we knew the results all along, and anyone could have predicted them (Caporael, Hartung). The only reply one can make is that everything is obvious in hindsight.

It is a benefit of being in the scientific community that theory and data can advance in a cumulative fashion, and that individual scientists can profit from the insight, commentary, and criticism of other scientists. In this Response, I try to take advantage of the insights provided by the 27 commentaries to consolidate the important research agenda that now lies ahead.

\section{Alternative hypotheses to account for sex differences}

No commentator provided alternative hypotheses for three of the five specific hypotheses and predictions advanced in the target article. No one proposed alternative explanations for the sex differences found across cultures in terms of (1) why males prefer younger mates, (2) why males value physical attractiveness more than females do, and (3) why males in many cultures value chastity more than females do (in no society are these sex differences significantly reversed). From this failure to suggest alternatives, I conclude that these internationally consistent sex differences are well accounted for by the evolutionary hypotheses outlined and not contested by the commentators.

In contrast, rival hypotheses were proposed to explain the greater value females placed on earning capacity and ambition-industriousness in their potential mates. These rival hypotheses take two forms:

1. The mate preference data result from or reflect "cultural norms" (with no indication of which features of cultures account for results or of how the norms originated; Zohar \& Guttman).

2. The effects are byproducts of the "culturally determined economic status of women" so that "male economic control makes male economic status critically important to females in selecting a mate" (Wallen; also Caporael and implicitly Glenn).

What is the status of the "cultural norm" hypothesis (1)? As stated, it outlines no specific features of cultures that could account for the results. It is hence unclear what data could confirm or falsify it. This sort of vague invocation of "culture" or "cultural norms" does not seem a useful alternative to the evolutionary explanation (see Tooby \& Cosmides).

The second hypothesis outlines a specific feature that is presumably characteristic of all societies and could account for the greater value females place on earning capacity across cultures: female economic powerlessness (as initially described by Buss \& Barnes 1986). This is a reasonable alternative because empirically testable propositions can be generated from it. For example: (1) As sex differences in economic power diminish, the sex difference in the value attached to earning power in mates should diminish; (2) within cultures, females who have greater economic power should value earning power in potential mates less than those who do not have economic power (i.e., they should show preferences more similar to those of men); (3) within cultures, males who are economically powerless should value earning capacity in potential mates more than those males who do have economic power (i.e., they should show preferences similar to those of women). Predictions (2) and (3) should be especially true if preferences are as highly contextdependent as Borgia, Dickemann, and Smuts assert.

I have assembled what data I could find with respect to these three predictions. Relative to the first prediction, Glenn provides at least indirect data by examining the correlation between the degree of development in countries and the magnitude of the sex difference in the importance they place on financial prospects and ambition-industriousness in mates. Although degree of development is inversely related to the absolute magnitude placed on these characteristics within a given sex, it is not significantly related to the magnitude of the sex difference observed on these economic variables.

A more direct index is provided by Stewart and Winter (1977), who developed reliable multiple-item indicators for a sample of countries of (a) economic equality between the sexes, and (b) social-educational equality between the sexes. Data were available for 30 of the 37 samples used in my study. The correlation across countries between degree of economic equality and male-female 
difference in the value assigned to earning capacity was -.22 (n.s.); that between social-educational equality and male-female difference in valuation of earning capacity was +.08 (n.s.). Further tests obviously need to be done. Nonetheless, these findings and those of Glenn so far provide no support for the hypothesis that degree of economic inequality is linked with magnitude of sex differences in the weight placed on earning power in potential mates.

To test predictions (2) and (3) from the economic powerlessness hypothesis, data collected in the United States on a sample of 100 males and 100 females were examined. Although not conducted specifically for this purpose, the study included measures of personal income, background socioeconomic status (SES), and a host of mate preference variables. To test predictions (2) and (3), correlations were computed between income and SES and each of four indices of mate preferences. These correlations are shown in Table 1.

As can be seen in Table 1 , women who make more money tend to value monetary and professional status of mates more than those who make less money, thus directly contradicting prediction (2). Male income is unrelated to mate preferences, providing no support for the hypothesis that economically powerless males will value earning prospects of a mate more than will men who make more money. Finally, SES tends to be positively correlated with the SES desired in a mate for both males and females. These results are consistent with those of Townsend (in press), who found that professional females (medical students) likely to be financially successful also valued financial considerations in potential mates more than did their male peers. Townsend concludes that "increasing women's socioeconomic status (SES) does not eliminate and may not even reduce traditional sex differences in mate selection criteria."

These various results obviously do not definitively refute the economic powerlessness hypothesis. Auxiliary hypotheses could be added. For example, perhaps females have been economically powerless for so long, with the resulting patterns of early socialization so deeply ingrained, that it will take years and extensive reeducation for mate preferences to reflect conditions of increasing economic equality between the sexes. These notions are plausible. However, to the degree that ad hoc auxiliary hypotheses have to be added to account for successive predictive failures, the credibility of the scien-

Table 1. Correlations between income and SES and mate preference variables

\begin{tabular}{|c|c|c|c|c|}
\hline \multirow{2}{*}{$\begin{array}{l}\text { Characteristics of } \\
\text { potential mate }\end{array}$} & \multicolumn{2}{|c|}{$\begin{array}{c}\text { Personal } \\
\text { income }\end{array}$} & \multicolumn{2}{|c|}{$\begin{array}{l}\text { Background } \\
\text { SES }\end{array}$} \\
\hline & Females & Males & Females & Males \\
\hline $\begin{array}{l}\text { Potential income of ideal } \\
\text { mate }\end{array}$ & $.31^{* * *}$ & .06 & -.02 & .10 \\
\hline Professional degree & $.35^{* * *}$ & .09 & -.09 & .00 \\
\hline College graduate & $.29^{* * *}$ & -.03 & .11 & $.26^{* *}$ \\
\hline SES of ideal mate & .11 & .10 & $.23^{*}$ & $.29 *$ \\
\hline
\end{tabular}

${ }^{*} p<.05 ;{ }^{* *} p<.01 ;{ }^{* *} p<.001$. tific hypothesis breaks down when compared with one that parsimoniously accounts for the data and requires no ad hoc auxiliary hypotheses. In sum, the available data support the evolutionary hypothesis and provide no support for the proximate economic powerlessness hypothesis.

As a final comment on alternative hypotheses, all versions of the "culture hypothesis" and "structural powerlessness" hypothesis leave a fundamental question unanswered: What is the origin of the economic inequality between males and females that is found so pervasively across cultures? An evolution-based model of intrasexual competition provides one potential answer. Members of one sex are expected to compete with each other to display characteristics sought in mates by members of the opposite sex (Buss 1988a). If females throughout human evolutionary history have placed greater value on external resources provided by mates, then there should have been greater selective pressure placed on males than on females to acquire those resources. Males who did so would be at a selective advantage over males who did not. This speculation obviously does not imply that what exists should exist (Caporael).

\section{The nature of psychological mechanisms}

The target article does not sufficiently distinguish between psychological mechanisms and proximate causes of those mechanisms. Fortunately, the commentaries of Symons and of Tooby \& Cosmides provide useful clarification (see also Buss, in press). As Symons notes, this study is important precisely because it provides evidence about psychological mechanisms in the form of mate preferences. The statement in the target article to the effect that we lack knowledge about proximate mechanisms should have been more precise: We lack knowledge about the ontogenetic causes of these psychological mechanisms.

Perhaps my failure to distinguish between psychological mechanisms and their ontogenetic causes led Caporael to argue against a position that I do not hold. I am in essential agreement with Caporael, Rushton, Russell \& Bartrip, Symons, and Tooby \& Cosmides that we must develop an evolutionary psychology and that this calls for a major focus on psychological mechanisms.

This focus leads to an exciting future agenda, but one whose outlines are far from clearly delineated. I suspect that it will include some of the psychological mechanisms of the sort proposed by Rancour-Laferriere, although an incisive description of such mechanisms may ultimately not map onto psychoanalytic categories. No doubt it will also include research on the physiological, neurological, and genetic substrates of such mechanisms, as suggested by Nyborg \& Boeggild and Rowe (although I would not endorse the extreme reductionism advocated by Nyborg \& Boeggild).

Food preferences may provide a useful analogue to mate preferences, although the latter are likely to be more complex. Humans appear to have species-typical proclivities to favor foods rich in fat and sugar while rejecting those that are bitter or sour. The fact that species-typical food preferences (i.e., mechanisms) exist at some level of description does not of course preclude 
powerful effects due to culture involving the particular food classes to which people are exposed. Nor do they preclude effects on actual consumption due to other variables, such as beliefs about what is good or healthful, parental pressures, or simple availability.

The target article (see also Buss et al., in press) documents both species-typical and sex-typical mate preferences. Like food preferences, these appear to vary to some degree with culture. Actual mating behavior, like food consumption, obviously varies with local beliefs, parental preferences, local availability of mates, and the nature of the mating system. The task for evolutionary psychologists is to identify psychological mechanisms and the adaptive problems they solve and to study overt behavior in the interaction of these mechanisms with important features of environments.

\section{Behavioral variability, flexibility, and context dependency}

Several commentators stress that human behavior is flexible and context-dependent, varying with important features of environments such as the nature of the mating system (Borgia, Dickemann, Nur, Smuts). Who can disagree? Mechanisms that produced invariant responses impervious to varying environmental contingencies would probably be selected against; and no one has proposed them in the case of human behavior. But how are we to understand human variability and flexibility?

The answer will come from many sources; suggestions that there is only one proper evolutionary approach (Smuts) must be resisted. An understanding of human variability will (in my view) depend heavily on the following three disparate areas: (1) an understanding of the nature of psychological mechanisms and the adaptive problems they solve (Caporael, Rushton, Symons, Tooby \& Cosmides), (2) an understanding of human genetic variability as it affects parameters of the psychological mechanisms (Rowe, Rushton, Zohar \& Guttman), and (3) an identification of important features of the environment (including structural, cultural, and resource features) that interact with given parameters of the psychological mechanisms to produce overt behavior.

Having said this, how can we make sense of the suggestion made by both Nur and Smuts (also implied by Dickemann) that evolutionary approaches should not include a search for mechanisms typical of a species or sex? An analogy to the study of anatomy is useful. The muscle mass of humans is known to be "context-dependent" in the way Smuts describes. Exercise increases muscle mass and inactivity decreases it. Furthermore, there are clear individual differences, some of them due to genetic differences. Finally, there may be cultures, environments, or mating systems that emphasize greater or lesser muscle mass. [See also Rushton "Genetic Similarity Theory, Human Altruism and Group Selection" BBS 12(3) 1989.]

Thus, there is variability due to individual contexts, genetic differences, and larger structural variables. Does this negate the fact that males and females differ "typically" in muscle mass, despite overlap of distributions? I would argue that it does not. The fact that behavior is often flexible and context-dependent does not contradict the existence of psychological mechanisms that are species-typical or sexually dimorphic.

\section{Is there a species-typical or sex-typical human nature?}

Each species has distinct physical and behavioral attributes that set it apart from other species as a result of unique selection histories. In many species, the sexes differ in consistent ways. Is it reasonable to attempt to characterize a "human nature" and to delineate the major ways in which human males and females differ? Despite large and important individual variation within our species and considerable overlap between males and females on most attributes, I agree with Symons and Tooby \& Cosmides that it is reasonable to approach a scientific description of our species-typical and sex-typical nature.

The data presented in the target article suggest two general conclusions: (1) All 37 societies in this study placed tremendous value on kindness-understanding and intelligence in potential mates; these characteristics were among the four most highly ranked in all 37 societies, and it is reasonable to characterize them, provisionally, as species-typical mate preferences; and (2) males and females in all (in the case of preferred age differences), or nearly all, societies showed significant differences in the value they attached to physical attractiveness (males valuing it more) and good financial capacity (females valuing it more).

These findings support Symons's (1987b) contention that human psychology is not sexually monomorphic. The current findings provide a baseline from which the study of human variability, its causes and consequences, can be studied. As Darwin (1871) noted more than a century ago, "No one disputes that the bull differs in disposition from the cow, the wild boar from the sow, the stallion from the mare. . . . Woman seems to differ from man in mental disposition" (p. 873).

\section{Methodological and data analytic issues}

Several commentators suggested alternative methods and raised issues about sampling, measurement, aggregation, and the use of particular statistical techniques.

Alternative methods. Several alternative methods and strategic approaches were suggested that complement in various ways the methods used in the target article. Bixler recommended the historical-comparative method, stressing the importance of including other species in comparative context. Carporael suggested generating hypotheses from engineering design considerations. Crawford proposed developing quantitative models of behavior-reproductive success links in ancestral populations and testing these models in current populations. Crawford also suggested testing evolutionary hypotheses against nonevolutionary hypotheses to document the unique power of evolutionary theory in the social sciences. These are all excellent suggestions; all their proponents are urged to pursue them.

Sampling issues. It is recommended by Crawford (see also Borgia and Essock) that my hypotheses be tested on 
more traditional societies such as the !Kung. Dickemann argues that the samples used are "biased" and "randomly chosen" and that this somehow obscures the "adaptive plasticity" that would be revealed otherwise. Irons, an eminent empirical anthropologist, disagrees with Dickemann, and argues that "the fact that these strategies do persistently manifest themselves in these novel [technologically sophisticated, less kin-oriented] environments indicates that the phenomena are very robust. [He] would be very surprised to discover that a comparable survey of preliterate societies did not reveal the same mate preferences."

In the target article, it is true that Western cultures have been disproportionately sampled compared with non-Western cultures. Does this sampling difference bias the results in favor of the key hypotheses? To examine this issue, I contrasted the non-Western samples (Nigeria, South African Zulu, Zambia, China, India, Indonesia, Iran, Palestinian Arab) with the Western samples. In terms of presence or absence of sex differences, the nonWestern samples were roughly comparable to the Western samples on all variables except chastity $-75 \%$ of the non-Western samples showed sex differences in the predicted direction, whereas slightly less than $50 \%$ of the Western samples showed this sex difference.

In terms of the magnitude of the sex differences, the two groups were roughly comparable on value attached to physical attractiveness, good earning capacity, and ambition-industriousness. These samples differed markedly, however, on the magnitude of the sex difference associated with relative age differences preferred between self and mate and with chastity preferences. Males in nonWestern cultures preferred mates who were 4.10 years younger on average, whereas males from Western cultures preferred mates who were only 2.22 years younger. On chastity, the average sex difference was more than twice as large in the non-Western samples as in the Western samples.

These results suggest that whatever sampling biases exist in the cultures represented in the target article, they appear to operate against the reproductive value and chastity hypotheses and to make little or no difference with respect to the other three hypotheses. These findings support Iron's suggestion that the persistence of these strategies in novel technologically sophisticated environments indicates that they are exceptionally robust.

Measurement issues. Questions are raised by Smuts and Dickemann about (a) the conjunction of "ambitious" and "industrious" into one variable, and (b) the meaning of terms such as "good looks." With respect to the first issue, one of the tests used was developed in the 1930s to study mate preferences. Although I agree with Smuts and Dickemann that future research should separate these two variables, they were used in combined form for a specific purpose: to provide an exact replication of prior studies conducted in the United States during the past 50 years so that mate preferences could be examined across generations within one culture, as well as across cultures.

Readers should consult Buss (1987), Cunningham (1986), and Symons (1979) for data and hypotheses about the constituents and interpretation of "good looks." It should be noted that although there are of course indi- vidual differences in the perception of what constitutes physical attractiveness, this variable shows a large degree of consensus among individuals that appears to transcend cultures (Berscheid \& Walster 1974; Cunningham 1986; Symons 1979).

Aggregation and disaggregation. It is suggested by Gladue and by Wallen that we aggregate the data by ethnic similarity, by religious similarity, or by common cultural traits. Dickemann, in contrast, suggests disaggregation by demographic transition. Dickemann appears to be under the mistaken impression that data from all 37 societies were aggregated prior to analysis. The data were in fact analyzed separately; each of the 37 samples was considered to represent an independent test of the key hypotheses.

The data can of course be aggregated in the ways suggested by Gladue and by Wallen; I am pleased to make this data set available to those who wish to conduct further analyses of this or another type. A specific hypothesis with a precise rationale would make arguments for these forms of aggregation more compelling.

Parametric statistics. A question is raised by Hartung about the use of $t$-tests, not on the grounds that the normality assumption may be violated (he admits that the $t$-test is "good about" this), but rather because he believes that females have greater variability than do males, thus violating the assumption of homogeneous variance. [See Benbon: "Sex Differences in Mathematical Reasoning Ability" BBS 11(2) 1988.] Hartung does not seem to be aware of four issues that directly pertain to this potential problem:

1. Possible violations of the homogeneity of variance assumption can be tested for. In this instance they were, and where unequal variances were found, separate variance estimates were used to calculate the $t$-tests.

2 . The ratio between the largest and smallest sample variance must deviate drastically from $1: 1$ (e.g., it should be greater than $20: 1$ ) for unequal variances to be a serious problem (Harris 1975); in no case did the ratio of male to female variances exceed $2.25: 1$, thus avoiding the danger zone by a factor of nearly 10 .

3. "We have such strong mathematical and empirical evidence of the robustness of [these] statistical procedures under violation of normality or homogeneity of variance assumptions that the burden of proof must be presumed to be on the shoulders of those who claim that a particular set of data can be analyzed only through "nonparametric' statistical techniques" (Harris 1975, p. 226).

4. Two parallel tests were used to test the key hypotheses, one based on a 4-point rating scale and one based on a 13-point ranking procedure. The potential truncation problem that might be encountered with the 4-point scale is surely less applicable to the 13-point rankings. It was so as to establish the generalizability of results across methods that these two different procedures were used to test the key hypotheses. Fortunately, the results of the two procedures were nearly identical, lending substantial credibility to the obtained findings.

Regarding the number of tests conducted, Hartung applies a formula for setting the $p$ value so that the collection of comparisons has a probability of a Type I error equal to alpha. It is true that as the number of tests 
increases, the probability of at least one spurious significant result also increases. However, "for planned orthogonal comparisons, contemporary practice in the behavioral sciences favors setting the Type I error probability at alpha for each comparison" (Kirk 1968, p. 78; see also Hays 1981; Winer 1971). The tests for sex differences in the target article are best viewed as independent replications, and the tests and $p$-values used are entirely appropriate.

Magnitude of effect. It is suggested by Caporael that because of the large sample sizes used, the sex differences may be statistically significant, but small in magnitude. To address this issue, the magnitude of effect was calculated for the target variables by computing a point biserial correlation between sex (male, female) and each of the five target variables. Across the 37 societies, the mean point-biserial correlations were as follows: good financial prospect (.34), ambition-industriousness (.20), good looks (.30), relative age difference preferred (.71), and chastity (.19). According to Cohen and Cohen (1975), a rough guide to the magnitude of effect in the social sciences is as follows: small (.10), medium (.30), and large (.50). Thus, the effect magnitude for chastity and ambition-industriousness can be viewed as small to medium; for good looks and good financial prospects, it is medium; and for relative age difference preferred between self and mate, it is large.

\section{Future research agenda}

Perhaps the most important insights to be gained from the 27 commentaries concern directions that future research might take to build upon the base of findings reported in the target article. In this section, I will discuss what I consider to be the most important of those directions.

Links to reproduction and actual mating behavior. The suggestion is made by Bixler, Gladue, Leonard, Wallen, and Zohar \& Guttman that we link the empirically documented mate preferences with actual reproductive behavior or reproductive success. The importance ascribed to links with current reproductive success hinges on the status of current mate preferences and raises the following questions: (1) Do these preferences represent psychological mechanisms that were adaptive in our evolutionary past? or (2) Do they represent a part of the current strategies individuals use to maximize fitness?

My reply to the first question is affirmative: Mate preferences represent adaptations forged during our environment of evolutionary adaptedness because of the reproductive benefits associated with those preferences in the past. From this perspective, links with current reproduction are certainly interesting, and empirical work should proceed to examine them. But current reproduction does not have any particular bearing on the viability of the conceptual framework within which they are interpreted (see Cosmides \& Tooby 1987; Symons 1987b; 1989). Environments change, and with them selection pressures change. Is it reasonable to require adaptive explanations of mechanisms to bear a current positive relationship with reproductive success? If we found that current variations in eyesight were not linked with differential reproductive success, would we conclude that keenness of vision was not an adaptation selected for in our evolutionary past? To try to link reproductive success with mate preferences in current populations is interesting and important, but it is not critical for testing the adaptive hypotheses about mate preferences that have been advanced by Darwin, Symons, Trivers, and Williams.

Zohar \& Guttman propose examining the links between preferences and actual mating decisions. As pointed out in the target article, mate preferences are expected to be one among a host of factors determining actual mating decisions. Parental preferences, preferences exerted by the opposite sex, personal characteristics, sex ratio, nature of the mating system, local availability of mates, and resources held may all be expected to play a role in actual mating decisions. In addition, mate preferences are many and complex; there may be tradeoffs and compromises - for example, accepting a lower threshold on one criterion in exchange for obtaining a greater fulfillment of another. In this sense, the target article is surely an oversimplified account of the complexity of mate preferences and their associations with other factors in determining actual mating decisions. The target article provides a base from which these complexities can be studied in greater detail and depth. Ethnographic studies (Irons), experimental research (Kenrick \& Keefe), and further cross-cultural research of the sort conducted here should all be part of this more intensive research effort.

Mate preference mechanisms and the environmental features that affect them. A second item on the research agenda should be an explicit exploration of the psychological mechanisms of mate preferences (Caporael, Symons, Tooby \& Cosmides), including their nature and environmental contingencies. The flexibility of these mechanisms probably depends on the degree of constancy of the selective advantages of the mate preferences in our evolutionary past that were documented in the target article. If the selective advantage to males of choosing fertile females were relatively invariant in our environment of evolutionary adaptedness, then large facultative shifts in such preferences would not be expected. If the selective advantage of preferences varied consistently with predictable features of the context, then contingent preferences would be more likely to have evolved. Currently, we do not know the important features of the environment that might affect shifts in mate preferences.

Several candidates for important environmental features have been suggested by the commentators. Barnard suggests the demographic features of sex ratio and the nature of the mating system. Glenn suggests and has empirically tested a hypothesis related to degree of development. Kenrick \& Keefe propose age of subject as a factor determining the age of preferred mate, and they present compelling data to back up their proposal. Lonner suggests comparing my data with those of Hofstede (1980) and Williams \& Best (1982) for possible insight into proximate influences such as work-related values and sex stereotypes.

The primary goal of the target article was not to examine these environmental variables but to focus on specific predictions about the importance of sex as a determinant of mate preferences. The data thereby si- 
multaneously provide information about the degree of variation in mate preferences both within sex and across societies. Now that the primary sex differences have been documented, the sources of variability due to factors other than biological sex should he explored.

Short- versus long-term mating contexts. A crucial distinction is pointed to by Kenrick \& Keefe and by Thornhill: short-term versus long-term mating strategies. Thornhill suggests that mate preferences might be facultative, especially with respect to male preferences for cues to fertility or reproductive value, depending on whether males are seeking short-term matings (fertility preferred) or long-term mateships (reproductive value preferred). Kenrick \& Keefe present fascinating data that males and females differ most in their preferences when short-term relationships are at stake, but show strong similarity of preferences when long-term matings are at stake.

This promising conceptual proposal deserves serious empirical exploration, both in the context of mate preferences and in other components of the mating system. For example, recent studies have focused on tactics used to attract mates; these tactics are presumed to depend in part on preferences exerted by the opposite sex (Buss, in preparation). The tactics of "acting provocative" and "wearing sexy clothes" were found (1) to show no sex differences in the effectiveness of attracting long-term mating partners, and (2) to show large and highly significant sex differences in the effectiveness of attracting short-tern mating partners; female use of these tactics was judged to be far more effective than male use. These data support Buss's (1988a) suggestion that short- versus long-term mating context is critical to understanding the tactics used to attract mates. Future research could focus on this critical distinction and its effect on moderating mate preferences and other components of human mating systems.

The evolution of intrasexual competition. Mate preferences represent only one component of human mating systems, albeit an important one. Intrasexual competition is another crucial component; future research could turn directly to the study of male-male and femalefemale competition. Ghiselin suggests that mate preferences do not involve pure reproductive competition and may hence be unrelated to sexual selection. Darwin (1871) and Fisher (1930), however, believed that mate preferences were integral to sexual selection.

Indeed, in his chapters on sexual selection in relation to man, Darwin (1871) anticipated the results of the target article in a particularly insightful manner in his book on sexual selection: "Preference on the part of the women steadily acting in any one direction, would ultimately affect the character of the tribe; for the women would generally choose not merely the handsomest men ... but those who at the same time were better able to defend and support them" (p. 903, emphasis added).

Darwin also recognized the important role that male preferences could have, noting that "women have long been selected for beauty ... . [and that] the continued preference by the men of each race for the more attractive women... will have tended to modify in the same manner all the individuals of both sexes belonging to the race" (1871, p. 901, emphasis added).

At another point, Darwin recognized that cross-character assortment (Buss 1985) would occur between resource-laden men and physically attractive women:

The strongest and most vigorous men - those who could best defend and hunt for their families, who . . . possessed the most property . . . would succeed in rearing a greater average number of offspring than the weaker and poorer members of the same tribes. There can, also, be no doubt that such men would generally be able to select the more attractive women. (1871, p. 899)

This hypothesis of cross-character assortment between men with resources and physically attractive females has been confirmed empirically in current populations by several independent investigators (Elder 1969; Taylor \& Glenn 1976; Udry \& Eckland 1984).

If mate preferences imposed by members of one sex do indeed have an effect on the nature of intrasexual competition, then several direct predictions follow about intrasexual competition for each sex. The findings of the target article suggest that for men intrasexual competition should center heavily on acquiring, displaying, and providing the resources that embody (part of) female mate preferences. Females should strive to appear youthful and physically attractive in intrasexual competition, because these represent important features of male mate preferences.

Furthermore, when deception occurs, it will be different for men and women. Men will exaggerate their external resource holdings or their willingness to devote those resources to a particular female. In contrast, women will exaggerate their youth, especially as they age beyond peak fertility, and their willingness to devote their reproductive value to a particular male. Finally, derogation of male competitors should involve putting down another's ability or willingness to provide external resources. Derogation of female competitors should center around a failure to possess high reproductive value or to deliver it to a particular male.

All these specific predictions about tactics of intrasexual competition can be derived from the current findings on mate preferences, combined with Darwin's (1871) articulation of the role of mate preferences in driving patterns of intrasexual competition. Some of these predictions have already been verified (Buss 1988a; 1988b; Buss \& Dedden, submitted). Further exploration of intrasexual competition across cultures is needed. The findings on mate preferences in the target article provide a basis for further study of the evolution of human intrasexual competition.

\section{Concluding comments}

The study of evolution and human behavior has suffered from a dearth of solid replicable findings to correspond to an abundance of theorizing and speculation. The target article provides a set of highly replicable findings that helps to balance the ratio of theory to data in the domain of human mating. According to Irons, the primary importance of this study is that (1) it has demonstrated the existence of sex differences in mate preferences across 


\section{References/Buss: Sex differences}

cultures that were not previously documented; (2) it confirms several important predictions from sexual selection theory; and (3) it illustrates the heuristic value of evolutionary theory in the study of human behavior.

The target article also adds to a small but steadily growing field of evolutionary psychology (e.g., Cosmides \& Tooby 1987; Shepard 1987; Staddon 1988; Symons 1989). Its central aim is discovering basic psychological mechanisms - forged by natural selection operating over thousands of generations - that exist because they successfully solved adaptive problems that humans had to confront to survive and reproduce. Selecting a mate is just one such problem, and the present results provide only a partial understanding of human mating mechanisms. The target article does document both speciestypical and sex-typical solutions to this problem, however, and in so doing takes us one step closer to understanding the evolutionary psychology of human mating.

\section{ACKNOWLEDGMENT}

The author extends special thanks to Laura Klem for statistical advice and to Arnold Buss and the Editor for improving the clarity of this Response.

\section{References}

Letters $a$ and $r$ appearing before authors' initials refer to target article and response respectively.

Alexander, R. D. (1974) The evolution of social behavior. Annual Review of Ecology and Systematics 5:325-83. [WI]

(1979) Darwinism and human affairs. University of Washington Press. [WI, RWS]

(1980) Darwinism and human affairs. Pitman. [CJB]

Alexander, R. D. \& Noonan, K. M. (1979) Concealment of ovulation, parental care, and human social evolution. In: Evolutionary biology and human social behavior: An anthropological perspective, ed. N. A. Chagnon \& E. G. Irons. Duxbury Press. [aDMB]

Anderson, C. M. (1986) Female age: Male preference and reproductive success in primates. International Joumal of Primatology 7:305-26. [aDMB, RHB]

Anderson, J. L. \& Crawford, C. B. (1988) Anorexia nervosa: Models of the disorder as an adaptation for reproductive suppression. Paper presented at meetings of the Canadian Psychological Association, Montreal, June. [CC]

Badcock, C. R. (forthcoming) Is the Oedipus Complex a Darwinian adaptation? Joumal of the American Academy of Psychoanalysis. [DR=L]

Bancroft, J., Sanders, D., Davidson, D. \& Warner, P. (1983) Mood, sexuality, hormones, and the menstrual cycle. III. Sexuality and the role of androgens. Psychosomatic Medicine 45:509-16. [HN]

Barron, F. (1963) Creativity and psychological health. Van Nostrand. [aDMB]

Bateman, A. J. (1948) Intrasexual selection in Drosophila. Heredity 2:34968. [aDMB]

Bateson, P. (1983) Mate choice. Cambridge University Press. [aDMB, MD]

Baucom, D. H., Besch, P. K. \& Callahan, S. (1985) Relation between testosterone concentration, sex role identity, and personality among females. Journal of Personality and Social Psychology 48(5):1218-26. [HN]

Berenstain, L. \& Wade, T. (1983) Intrasexual selection and male mating strategies in baboons and macaques. International Journal of Primatology 4:201-35. [aDMB, RHB]

Berscheid, E. (1985) Interpersonal attraction. In: Handbook of social psychology, vol. 2, ed. G. Lindzey \& E. Aronson. Random House/Erlbaum. [DTK]

Berscheid, E. \& Walster, E. (1974) Physical attractiveness. In: Advances in experimental social psychology, ed. L. Berkowitz. Academic Press. [rDMB]

Betzig, L. L., Borgerhoff Mulder, M. \& Turke, P. W. (1988) Human reproductive behavior. Cambridge University Press. [aDMB]

Blumberg, R. L. \& Winch, R. F. (1972) Societal complexity and familial complexity: Evidence for the curvilinear hypothesis. American Journal of Sociology 77:898-920. [NDG]

Borgerhoff Mulder, M. (1988) Kipsigis bridewealth payments. In: Human reproductive behavior: A Darwinian perspective, ed. L. L. Betzig, M. Borgerhoff Mulder \& P. W. Turke. Cambridge University Press. [aDMB, MD]

Bradbury, J. W. \& Anderson, M. (1987) Sexual selection: Testing the alternatives. Wiley. [NN]

Brehm, S. S. (1985) Intimate relationships. Random House. [DTK]

Burgess, E. W. \& Wallin, P. (1953) Engagement and marriage. Lippincott. [AZ]

Burian, R. M. (1983) Adaptation. In: Dimensions of Darwinism, ed. M. Grene. Cambridge University Press. [DS]

Burley, N. (I983) The meaning of assortative mating. Ethology and Sociobiology 4:191-203. [JPR]

Buss, D. M. (1984) Evolutionary biology and personality psychology: Toward a conception of human nature and individual differences. American Psychologist 39:1135-47, [DCR]

(1985) Human mate selection. American Scientist 73:47-51. [aDMB, JPR] (1987) Sex differences in human mate selection criteria: An evolutionary perspective. In: Sociobiology and psychology: Ideas, issues, and applications, ed. C. Crawford, M. Smith \& D. Krebs. Erlbaum [aDMB]

(1988a) The evolution of human intrasexual competition: Tactics of mate attraction. Journal of Personality and Social Psychology 54:616-28. [arDMB, NWT]

(1988b) From vigilance to violence: Tactics of mate retention in American undergraduates. Ethology and Sociobiology 9:291-317. [arDMB]

(in press) Preference mechanisms in human mating. In: Evolved mechanisms, ed. J. Barkow, L. Cosmides \& J. Tooby. Cambridge University Press. [rDMB]

(in preparation) Short- and long-term mating: Tactics. [rDMB]

Buss, D. M. \& Barnes, M. F. (1986) Preferences in human mate selection Journal of Personality and Social Psychology 50:559-70. [aDMB, AZ RHB, LRC]

Buss, D. M. \& Dedden, L. (submitted) Derogation of competitors. [aDMB] Buss, D. M., et al. (in press) International preferences in selecting mates: $A$ study of 37 cultures. Journal of Cross-cultural Psychology. [rDMB]

Calder, C. (1967) Breeding behavior of the roadrunner Geococcyx californianus. Aux 84:597-98. [aDMB]

Caporael, L. R., Dawes, R. M., Orbell, J. M. \& van de Kragt, A. J. C (submitted) Selfishness examined: Cooperation in the absence of egoistic incentives. [LRC]

Chagnon, N. A. (1983) Yanomamo: The fierce people (3rd ed.). Holt, Rinehart and Winston. [DS]

Cohen, J. \& Cohen, P. (1975) Applied multiple regression/correlation analysis for the behavioral sciences. Erlbaum. [rDMB]

Cosmides, L. \& Tooby, J. (1987) From evolution to behavior: Evolutionary psychology as the missing link. In: The latest on the best: Essays on evolution and optimality, ed. J. Dupré. MIT Press. IrDMB, CC, DS JT]

Crawford, C. B. \& Anderson, J. L. (1988) Testing evolutionary hypotheses about pathological behaviors. Paper presented at meetings of the Canadian Psychological Association, Montreal, June. [CC]

Cunningham, M. R. (1986) Measuring the physical in physical attractiveness: Quasi-experiments on the sociobiology of female beauty. Jourmal of Personality and Social Psychology 50:925-35. [rDMB]

(1988) Sociobiological dynamics in facial attractiveness. Paper presented at the 96th Annual Convention of the American Psychological Association, Atlanta. [RHB]

Daly, M. \& Wilson, M. (1983) Sex, evolution, and behavior. Willard Grant Press. [aDMB, CJB, MD, DTK, RWS]

(1988) Homicide. Aldine de Gruyter. [aDMB]

Daly, M., Wilson, M. \& Weghorst, S. J. (1982) Male sexual jealousy. Ethology and Sociobiology 3:11-27. [aDMB]

Darwin, C. (1859) On the origin of the species by means of natural selection, or preservation of favoured races in the struggle for life. London: Murray. [aDMB, MTG]

(1859a) The origin of species by means of natural selection. Penguin Books. [RHB]

(1871) The descent of man and selection in relation to sex. London: Murray. [aDMB]

(1877) The descent of man and selection in relation to sex, second edition, revised and augmented. Murray. [JLL]

Darwin, E. (1794) Zoonomia; or, the laws of organic life, vol. 1. J Johnson. [MTG]

de Waal, F. (1982) Chimpanzee politics. Harper \& Row. [HRB]

Dickemann, M. (1979a) Female infanticide, reproductive strategies and social 
stratification: A preliminary model. In: Evolutionary biology and human social behavior: An anthropological perspective, ed. N. A. Chagnon \& W. D. Irons. Duxbury Press. [MD]

(1979b) The ecology of mating systems in hypergynous dowry societies. Social Science Information 18:163-75. [MD]

(1981) Paternal confidence and dowry competition: A biocultural analysis of purdah. In: Natural selection and social behavior, ed. R. D. Alexander \& D. W. Tinkle. Chiron Press. [aDMB, MD]

(1985) Human sociobiology: The first decade. New Scientist 107:38-42. [C]B]

Dunbar, K. I. M. (1982) Intraspecific variations in mating strategy. In Perspectives in ethology, vol. 5, ed. P. P. G. Bateson \& P. H. Klopfer. Plenum. [NN]

Eberhard, W. G. (1985) Sexual selection and animal genitalia. Harvard University Press. [MTG]

Eibl Eibesfeldt, I. (1970) Ethology: The biology of behavior. Holt, Rinehart and Winston. [NN]

Elder, G. H., Jr. (1969) Appearance and education in marriage mobility. American Sociological Review 34:519-33. [rDMB]

Ellis, B. J. (in press) The evolution of sexual attraction: Evaluative mechanisms in women. In: Evolved mechanisms in human behavior and culture, ed. J. H. Barkow, L. Cosmides \& J. Tooby. Oxford University Press. [aDMB]

Duby, G. (1978) Medieval marriage: Two models from 12th-century France, trans. E. Forster. Johns Hopkins University Press. [MD]

Emlen, S. T. \& Oring, L. W. (1977) Ecology, sexual selection, and the evolution of mating systems. Scicnce 197:215-30. [aDMB]

Epstein, E. \& Guttman, R. (1984) Mate selection in man: Evidence, theory and outcome. Social Biology 31:3-4. [AZ]

Fisher, R. A. (1930) The genetical theory of natural selection. Clarendon Press. [aDMB, CC, MTG]

Fisher, S. (1973) The female orgasm: Psychology, physiology, fantasy. Basic Books. $[\mathrm{DR}=\mathrm{L}]$

Flinn, M. (1981) Uterine vs. agnatic kinship variability and associated cousin marriage preferences: An evolutionary biological analysis. In: Natural selection and social behavior; Recent research and new theory. ed. R. D Alexander \& D. W. Tinkle. Chiron Press. [GB, WI]

(1988) Mate guarding in a Caribbean village. Ethology and Sociobiology 9:1-28. [aDMB, MD]

Flinn, M. V. \& Low, B. (1986) Resource distribution, social competition, and mating patterns in human societies. In: Ecological aspects of evolution: Birds and mammals, ed. D. 1. Rubinstein \& R. W. Wrangham. Princeton University Press. [MD]

Foley, R. (1987) Another unique species: Patterns in human evolutionary ecology. John Wiley. [LRC]

Ford, C. S. \& Beach, F. A. (1951) Patterns of sexual behavior. Harper \& Row. [aDMB, JLL]

Freedman, D. G. (1979) Human sociobiology. Free Press. [RHB]

Gardner, E., Gray, D. J. \& O'Rahilly, R. O. (1975) Anatomy. W. B. Saunders. [JLL]

Gaulin, S. J. C. \& Schlegel, A. (1980) Paternal confidence and paternal investment: A cross-cultural test of a sociobiological hypothesis. Ethology and Sociobiology 1:301-9 [WI]

Ghiselin, M. T. (1969) The triumph of the Darwinian method. University of California Press. [MTG]

(1974) The economy of nature and the evolution of sex. University of California Press. [MTG]

Glass, G. V. \& Stanley, J. C. (1970) Statistical methods in education and psychology. Prentice-Hall. [JH]

Goodall, J. (1986) The chimpanzees of the Gombe. Belknap Press. [RHB]

Gough, H. G. (1973) Personality assessment in the study of population. In Psychological perspectives on population, ed. J. T. Fawcett. Basic Books. [aDMB]

Gould, S. J. (1986) Evolution and the triumph of homology, or why history matters. American Scientist 74:60-69. [RHB]

Goody, J. (1983) The development of the family and marriage in Europe. Cambridge University Press. [MD]

Guttman, L. (1981) Efficacy coefficients for differences among averages. In: Multidimensional data representations: When $b$ why, ed. I. Borg. Mathesis Press. [AZ]

(1988) Eta, Disco, O Disco and F. Psychometrika 53. [AZ]

Hamilton, W. D. (1964) The evolution of social behavior. Journal of Theoretical Biology 7:1-52. [RWS]

(1966) The moulding of senescence by natural selection. Journal of Theoretical Biology 12:12-45. [RWS]

(1971) Selection of selfish and altruistic behavior in some extreme models. In: Man and beast: Comparative social behavior, ed. J. F. Eisenberg \& W. S. Dillon. Smithsonian Institution. [NN]
Harding, S. \& O'Barr, J. F. (1987) Sex and scientific inquiry. University of Chicago Press. [LRC]

Harris, R. J. (1975) A primer of multivariate statistics. Academic Press. [rDMB]

Hart, C. W. \& Pilling, A. R. (1960) The Tiwi of North Australia. Holt, Rinehart \& Winston. [aDMB, MD]

Hays, W. L. (1981) Statistics, third edition. Holt, Rinehart \& Winston. [rDMB]

Hill, R. (1945) Campus values in mate selection. Journal of Home Economics 37:554-58. [aDMB]

Hofstede, G. (1980) Culture's consequences: International differences in workrelated values. Sage Publications. [WJL]

Hrdy, S. B. (1981) The woman that never evolved. Harvard University Press. [RHB]

Hudson, J. W. \& Henze, L. F. (1969) Campus values in mate selection: A replication. Joumal of Marriage and the Family 31:772-75. [aDMB]

Huntingford, F. A. \& Turner, A. (1987) Animal conflict. Chapman and Hall. [CJB]

Imperato-McGinley, J., Guerrero, L., Gautier, T. \& Peterson, R. E. (1974) Steroid 5-alpha-reductase deficiency in man: An inherited form of malc pseudohermaphroditism. Science 186:1213-15. [HN]

Irons, W. (1983) Human female reproductive strategies. In: Social behavior of female vertebrates, ed. S. K. Wasser. Academic Press. [MD, WI]

Jencks, C. (1979) Who gets ahead? The determinants of economic success in America. Basic Books. [aDMB]

Jowell, R. \& Witherspoon, S. (1987) British social attitudes. Gower. [CJB]

Katchadourian, H. (1977) The biology of adolescence. W. H. Freeman. [JLL]

Kenrick, D. T. (1987) Gender, genes, and the social environment: A biosocial interactionist perspective. In: Review of personality and social psychology: Sex and gender, vol. 7, ed. P. Shaver \& C. Hendrick. Sage. [DTK]

Kenrick, D. T. \& Keefe, R. C. (submitted) Gender differences in age criteria for a mate increase over the life span. [DTK]

Kenrick, D. T., Sadalla, E. K., Groth, G. \& Trost, M. R. (in press) Gender and trait requirements in a mate: An evolutionary bridge between personality and social psychology. Journal of Personality. [DTK]

Kenrick, D. T. \& Trost, M. R. (1988) A reproductive exchange model of heterosexual relationships: Putting proximate economics in ultimate perspective. In: Review of personality and social psychology, vol. 10, ed. C. Hendrick. Sage. [DTK, NWT]

Kimura, D. (1987) Are man's and woman's brains really different? Canadian Psychology 28:133-47. [CC]

Kinsey, A. C., Pomeroy, W. B. \& Martin, C. E. (1948) Sexual behavior in the human male. Saunders. [DTK]

Kirk, R. (1968) Experimental design: Procedures for the behavior sciences. Brooks-Cole. [rDMB]

Kitcher, P. (1985) Vaulting ambition: Sociobiology and the quest for human nature, MIT Press. [LRC]

Kurian, G. (1979) Cross-cultural perspectives of mate-selection and marriage. ed. G. Kurian. Greenwood. [aDMB]

Lack, D. (1940) Pair formation in birds. Condor 42:269-86. [aDMB]

Lambert, H. H. (1987) Biology and equality: A perspective on sex differences. In: Sex and scientific inquiry, ed. S. Harding \& J. F. O'Barr. University of Chicago Press. [LRC]

Lee, G. R. (1982) Family structure and interaction: A comparative analysis. University of Minnesota Press. [NDG]

Lenington, S. (1981) Child abuse: The limits of sociobiology. Ethology and Saciobiology 2:17-29. [CJB]

Levi-Strauss, C. (1969) The elementary structures of kinship. Revised edition, trans. J. H. Bell, J. R. von Sturmer \& R. Needham. Beacon Press. [MD]

Littlefield, C. H. \& Rushton, J. P. (1986) When a child dies: The sociobiology of bereavement. Journal of Personality and Social Psychology 51:797802. [JPR]

Low, B. S. (1979) Sexual selection and human ornamentation. In: Evolutionary biology and human social behavior, ed. N. A. Chagnon \& W. Irons. Duxbury. [JLL]

Lumsden, C. J. \& Wilson, E. O. (1981) Genes, mind, and culture. Harvard University Press. [RJHR]

(1982) Precis of Genes, mind and culture. Behavioral and Brain Sciences 5:1-37. [JPR]

Maccoby, E. \& Jacklin, C. (1974) The psychology of sex differences. Stanford University Press. [aDMB, LRC, WI]

Majerus, M. (1986) The genetics and evolution of female choice. Trends in Ecology and Evolution 1:1-7. [aDMB]

Maynard Smith, J. (1977) Parental investment - a prospective analysis. Animal Behavior 25:1-9. [NN] 
(1982) Evolution and the theory of games. Cambridge University Press [NN]

Maynard Smith, J. \& Price, G. R. (1973) The logic of animal conflict. Nature 246:15-18. [NN]

Mayr, E. (1976) Behavior programs and evolutionary strategies. In: Evolution and the diversity of life: Selected essays, ed. E. Mayr. Harvard University Press. [JT]

(1982) The growth of biological thought. Belknap Press. [RHB]

(1983) How to carry out the adaptationist program. American Naturalist 121:324-34. [RHB]

McDougall, W. (1908) Social psychology. Methuen. [DTK]

McGinnis, R. (1958) Campus values in mate selection. Social Forces 35:36873. [aDMB]

Mead, M. (1949) Male and female. Morrow. [JT]

Meyer-Bahlberg, H. F. L. (1984) Psychoneuroendocrine research on sexual orientation: Current status and future options. In: Sex differences in the broin Progress in the brain research, vol. 61, ed. G. J. de Vries, J. P. C. de Bruin, H. B. M. Uylings \& M. A. Corner. Elsevier Biomedical. [HN]

Money, J. \& Ehrhardt, A. A. (1972) Man and woman, boy and girl. Johns Hopkins University Press. [HN]

Murdock, G. P. (1949) Social structure. Macmillan. [MD]

(1967) Ethnographic atlas. Pittsburgh University Press. [aDMB, SME]

Murstein, B. I. (1986) Paths to marriage. Sage Publications. [aDMB]

Nisbet, I. C. T. (1973) Courtship feeding, egg-size and breeding success in common terms. Nature 241:141-42. [aDMB]

Nunally, J. (1978) Psychometric theory. McGraw-Hill. [aDMB, JH]

Nyborg, H. (1979) Sex chromosome abnormalities and cognitive performance. V: Female sex hormone and discontinuous cognitive development. Paper presented at the symposium on Cognitive Studies at the fifth biennial meeting of the International Society for the Study of Behavioural Development, Lund, Sweden. [HN]

(1983) Spatial ability in men and women: Review and new theory. In: Advances in human research and therapy, vol. 5 (whole number), Monography Series. Pergamon Press. [HN]

(1984) Performance and intelligence in hormonally-different groups. In: Ser differences in the brain: Progress in the brain research, vol. 61, ed. G. J de Vries, J. P. C. de Bruin, H. B. M. Uylings \& M. A. Corner. Elsevier Biomedical Press. [HN]

(1986) Sex chromosomes, sex hormones, and developmental disturbances: In search of a model. Paper presented at the 152nd annual national meeting of the American Association for the Advancement of Science, Philadelphia. [HN]

(1987) Covariant trait development across species, race, and within individuals: Differential K-theory, genes, and hormones? Paper presented at the 3rd meeting of the International Society for the Study of Individual Differences, Toronto, Canada. [HN]

(in preparation) Sex, body, mind, and society: A physicological approach. [HN]

O'Kelly, C. G. \& Carney, L. S. (1986) Women and men in society: Crosscultural perspectives on gender stratification. Wadsworth Publishing. [aDMB]

OPCS (1988) Mid 1985-based subnational population projection 1985-2005 London, Report of the Office of Population Censuses and Surveys, PP3 Series No. 7. [CJB]

Parker, S. T. (1987) A sexual selection model for hominid evolution. Human Evolution 2:235-53.

Partridge, L. \& Halliday, T. (1984) Mating patterns and mate choice. In: Behovioural ecology: An evolutionary approach, 2nd ed., ed. J. R. Krebs \& N. B. Davies. Sinauer. [NN]

Pfafflin, S. M. (1984) Women, science, and technology. American Psychologist 39:1183-86. [LRC]

Plomin, R., DeFries, J. C. \& McClearn, G. E. (1980) Behavioral genetics: A primer. W. H. Freeman. [DCR]

Pomiankowski, A. (1987) Sexual selection: The handicap principle does work sometimes. Proceedings of the Royal Society of London Series B 231:12345. $[\mathrm{NN}]$

Price, T., Kirkpatrick, M. \& Arnold, S. J. (1988) Directional selection and the evolution of breeding date in birds. Science 240:798-99. [DRC]

Purifoy, F. E. \& Koopmans, L. H. (1981) Androstenedione, testosterone, and free testosterone concentration in women of various occupations. Social Biology 26(3):179-88. [HN]

Rancour-Laferrier, D. (1985) Signs of the flesh: An essay on the evolution of hominid sexuality. Berlin: Mouton de Gruyter. [DR-L]

Republic of China (1987) 1986 Taiwan-Fukien demographic fact book: Republic of China. Ministry of the Interior, Taipei, Taiwan. [aDMB]

Rice, W. R. (1988) Heritable variation in fitness as a prerequisite for adaptive female choice: The effect of mutation-selection balance. Evolution 42:817-20.
Richardson, H. (1939) Studies of mental resemblance between husbands and wives and between friends. Psychological Bulletin 36:104-42. [R]HR]

Robinson, J. (1982) Intrasexual competition and mate choice in primates. American Journal of Primatology Suppl. 1:131-44. [aDMB]

Rohde, V., Stahl, F., Goertz, F. \& Dörner, G. (1978) Neuroendocrine findings in subjects with sexual deviations. In: Hormones and brain development: Developments in endocrinology, vol. 3, ed. G. Dörner \& M. Kawakami. Elsevier/North-Holland Biomedical Press. [HN]

Romanes, G. J. (1893-97) Darwin and after Daruin, 2nd ed., 3 vols. Longmans. [MTG]

Rowe, D. C., Clapp, M. \& Wallis, J. (1987) Physical attractiveness and the similarity of identical twins. Behacior Genetics 17:191-201. [DRC]

Rowe, D. C. \& Osgood, D. W. (1984) Heredity and sociological theories of delinquency: A reconsideration. American Sociological Recicw 49:52640. [JPR]

Rushton, J. P. (1988) Genetic similarity, mate choice, and fecundity in humans. Ethology and Sociobiology 9:329-35. [JPR]

Rushton, J. P. \& Bogaert, A. (1987) Race differences in sexual behavior: Testing an evolutionary hypothesis. Journal of Rescarch in Personality 21:529-51. [HN]

Rushton, J. P. \& Chan, P. K. F. (in press) Genetic similarity in male friendships. Ethology and Sociobiology. [JPR]

Rushton, J. P. \& Nicholson, I. R. (1988) Genetic similarity theory, intelligence, and human mate choice. Ethology and Sociobiology 9:4557. [JPH]

Rushton, J. P. \& Russell, R. J. H. (1985) Genetic similarity theory: A reply to Mealey and new evidence. Behatiour Genetics 15:575-82. [JPR]

Rushton, J. P., Russell, R. J. H. \& Wells, P. A. (1984) Genetic similarity theory: Beyond kin selection. Behatior Gcnetics 14:179-93. [aDMB, JPR]

Russell, R. J. H., Wells, P. A. \& Rushton, J. P. (1985) Evidence for genetic similarity detection in human marriage. Ethology and Sociobiology 6:18387. [RJHR, JPR]

Sahlins, M. (1976) The use and abuse of biology: An anthropological critique of sociobiology. University of Michigan Press. [JT]

Scarr, S. \& Grajeck, S. (1982) Similarities and differences among siblings. In: Sibling relationships, ed. M. E. Lamb \& B. Sutton-Smith. Erlbaum. [JPR]

Schiavi, R. C., Theilgaard, A., Owen, D. K. \& White, D. (1984) Sex chromosome anomalies, hormones, and aggressivity. Archices of Gcneral Psychiatry 41:93-99. [HN]

Segalen, M. (1986) Historical anthropology of the family, trans. J. C. Whitehouse \& S. Matthews. Cambridge University Press. [MD]

Shepard, R. N. (1987) Evolution of a mesh between principles of the minds and regularities of the world. In: The latest on the best: Essays on evolution and optimality, ed. J. Dupre. MIT Press. [rDMB]

Sherwin, B. B. \& Gelfand, M. M. (1987) The role of androgen in the maintenance of sexual functioning in oophorectomized women. Psychosomatic Medicine 49:397-409. [HN]

Sherwin, B. B., Gelfand, M. M. \& Brender, W. (1985) Androgen enhances sexual motivation in females: A prospective, crossover study of sex steroid administration in the surgical menopause. Psychosomatic Mcdicine 47(4):339-51. [HN]

Shields, W. M. \& Shields, L. M. (1983) Forcible rape: An cvolutionary perspective. Ethology and Sociobiology 4:115-35. [CJB]

Short, R. (1976) The evolution of human reproduction. Proccedings of the Royal Society of London 195:3-24. [aDMB]

Simpson, G. G. (1964) This tiew of life. Harcourt Brace. [HRB]

Smith, R. L. (1984) Human sperm competition. In: Sperm competition and the evolution of animal mating systcms, ed. R. L. Smith. Academic. [JLL]

Smuts, B. (1987) Sexual competition and mate choice. In: Primate socictics, ed. B. Smuts et al. University of Chicago Press. [aDMB]

Soma, H., Takayama, M., Takashi, K., Akaeda, T. \& Takoro, K. (1975) Serum gonadotropin levels in Japanese women. Obstetrics and Gynecology 46(3):311-12. [HN]

Sperber, D. (1984) Anthropology and psychology: Towards an epidemiology of representations. Man 20:73-89. [JT]

Spuhler, J. N. (1968) Assortative mating with respect to physical characteristics. Eugenics Quarterly 15:128-40. [RJHR]

Staddon, J. E. R. (1988) Learning as inference. In: Evolution and lcarning, ed. R. C. Bolles \& M. D. Beecher. Erlbaum.

Stewart, A. J. \& Winter, D. G. (1977) The nature and causes of fermale suppression. Signs 2:531-53. [rDMB]

Stone, L. (1977) The family, sex and marriage in England: 1500-1800. Harper \& Row. [MD]

Symons, D. (1979) The evolution of human sexuality. Oxford University Press. [aDMB, DS] 


\section{References/Buss: Sex differences}

(1980) Precis of The evolution of human sexuality. Behacioral and Brain Sciences 3:171-214. [JPR]

(1987a) Can Darwin's view of life shed light on human sexuality? In

Approaches and paradigms in human sexuality, ed. J. H. Geer \& W. T.

O'Donohue. Plenum. [aDMB]

(1987b) If we're all Darwinians, what's the fuss about? In: Sociobiology and psychology: Idcas, issucs, and applications, ed. C. Crawford, M. Smith \&

D. Krebs. Erlbaum. [arDMB, DS, JT]

(1989) A critique of Darwinian anthropology. Ethology and Sociobiology 9(5). [rDMB, JT, DS]

Taylor, P. A. \& Clenn, N. D. (1976) The utility of education and attractiveness for femles' status attainment through marriage. American Sociological Review 41:484-98. [rDMB]

Tellegen, A., Lykken, D. T., Bouchard, T. J., Jr., Wilcox, K., Segal, N. \& Rich, S. (1988) Personality similarity in twins reared apart and together. Journal of Personality and Social Psychology 54:1031-39. [JPR]

Theilgaard, A. (1984) A psychological study of the personalities of XYY. and XXY-men. Acta Psychiatrica Scandinavica 69 (suppl. no. 315). [HN]

Thiessen, D. D. \& Gregg, B. (1980) Human assortative mating and genetic equilibrium. Ethology and Sociobiology 1:111-40. [aDMB]

Thomhill, R. \& Thomhill, N. W. (1983) Human rape: An evolutionary analysis. Ethology and Sociobiology 4:63-99. [aDMB, NWT]

Tomlinson, I. P. M. (1988) Diploid models of the handicap principle. Heredity 60:283-93.

Tooby, J. \& Cosmides, L. (1989) Evolutionary psychology and the generation of culture, part 1: Theoretical considerations. Ethology and Sociobiology $9(5)$. [DS, JT]

(submitted) On the universality of human nature and the uniqueness of the individual: The role of genetics and adaptation. [JT]

Townsend, J. M. (in press) Mate selection criteria: A pilot study. Ethology and Sociobiology. [rDMB]

Trivers, R. L. (1972) Parental investment and sexual selection. In: Sexual selection and the descent of man, ed. B. Campbell. Aldine. [aDMB, DTK, CJB, JLL, RWS]

(1985) Social ecolution. Benjamin/Cummings. [aDMB, CC, RWS]

U. S. Department of Commerce (1987) Statistical abstract of the United States: 1987. U. S. Government Printing Office. [NDG]

Uddenberg, N. (1974) Psychological aspects of sexual inadequacy in women. Journal of Psychosomatic rescarch 18:33-47. [DR-L]
Udry, J. R. \& Eckland, B. K. (1984) Benefits of being attractive: Differential payoffs for men and women. Psychological Reports 54:47-56. [rDMB]

United Nations (1988) 1986 demographic yearbook. United Nations. [aDMB]

Vandenberg, S. G. (1972) Assortative mating, or who marries whom? Behavior Genetics 2:127-57. [RJ HR]

van den Berghe, P. L. (1983) Human inbreeding avoidance: Culture in nature. Behavioral and Brain Sciences 6:91-123. [JPR]

Vining, D. R. (1984) Subfertility among the very intelligent: An examination of the American Mensa. Personality and Individual Differences 5(6):72533. [HN]

Wallace, A. R. (1870) Contributions to the theory of natural selection. Macmillan. [MTG]

Weismann, A. (1891-92) Essays upon heredity and kindred subjects, 2nd ed., 2 vols. Clarendon Press. [MTG]

West-Eberhard, M. J. (1983) Sexual selection, social competition and speciation. Quarterly Review of Biology 58:155-83. [MTC]

(1987) Flexible strategy and social evolution. In: Animal societies: Theories and facts, ed. Itô, Y., Brown, J. L. \& Kikkawa, J. Japan Science Society Press. [RWS]

Whalen, R. E. (1980) Evolution causation: How proximate is ultimate. Behavioral and Brain Sciences 3:202-3. [BAC]

Willerman, $L$. (1979) The psychology of individual and group differences. Freeman. [aDMB]

Williams, G. C. (1957) Pleiotropy, natural selection and the evolution of senescence. Evolution 11:398-411. [RWS]

(1975) Sex and evolution. Princeton University Press. [aDMB, JH]

Williams, J. E. \& Best, D. L. (1982) Measuring sex stereotypes: A thirtynation study. Sage Publications. [W]L]

Wilson, E. O. (1975) Sociobiology: The new synthesis. Harvard University Press. [CJB]

(1978) On human nature. Harvard University Press. [CJB]

Winer, B. J. (1971) Statistical principles in experimental design (2nd edition). McGraw-Hill. [rDMB]

Wittenburger, J. L. (1981) Animal social behavior. Duxbury Press. [CC]

Wrangham, R. W. (1988) Bridging the gaps: Social relationships in animals and people. Rackham Reports 1987-1988, University of Michigan. [RWS]

Whyte, M. K. (1978) The status of women in preindustrial societies. Princeton University Press. [MD] 


\section{Journal of EXPERIMENTAL AND THEORETICAL ARTIFICIAL INTELLIGENCE}

\section{EDITORS}

Eric Dietrich

Dept of Philosophy, Program in Philosophy

and Computer \& Systems Sciences

State University of New York

Binghampton NY 13901, USA

Chris Fields

Computing Research Laboratory

New Mexico State University

Box 3CRL

Las Cruces NM 88003-0001, USA

The aim of JOURNAL OF EXPERIMENTAL AND THEORETICAL ARTIFICIAL INTELLIGENCE (JETAI) is to advance scientific research in artificial intelligence (AI) by providing a public forum for the presentation, evaluation and criticism of research results, the discussion of methodological issues, and the communication of positions, preliminary findings and research directions. Work in all subfields of $\mathrm{AI}$ research, including work on problem solving, perception, learning, knowledge representation, memory and neural system modelling will be within the scope of JETAI.

- Through editorial statements and editorial policy, JETAI will encourage AI research that adopts a scientific rather than engineering methodology.

- JETAI will provide a forum for an active and lively discussion of foundational and methodological issues in $A I$ research and for critical discussions of results and techniques published either in JETAI or elsewhere in the AI or cognitive science literature.

- JETAI will publish the broad range of AI research quickly - the Editors will strive to maintain a three month turnaround time between submission and a publication decision.

\section{Contents of inaugural issues}

Minds, Machines and Searle, Stevan Harnad - Explanation-based Learning, B. J. Krawchuk and I. A. Witten - Recognition and Classification of Concepts, F. Gomez and C. Segami

- Exploring the No-function-in-structure Principle, A. Keuneke and D. Allemang.

Publisher: Taylor \& Francis

Subscription Information

Volume 1 (1989)

Institutional Rate:

$$
\text { US\$120 E65 }
$$

Personal Rate: US\$45 £30

\section{SEND FOR A FREE SPECIMEN COPY}

TAYLOR \& FRANCIS

UK: Rankine Road, Basingstoke, Hants RG24 OPR

USA: 242 Cherry Street, Philadelphia PA 19106-1906 